\title{
An efficient, conservative, time-implicit solver for the fully kinetic arbitrary-species 1D-2V Vlasov-Ampère system
}

\author{
S. E. Anderson ${ }^{\mathrm{a}, *}$, W. T. Taitano ${ }^{\mathrm{a}}$, L. Chacón ${ }^{\mathrm{a}}$, A. N. Simakov ${ }^{\mathrm{b}}$ \\ ${ }^{a}$ Theoretical Division, Los Alamos National Laboratory, Los Alamos, NM, U.S.A. 87545 \\ ${ }^{b}$ Theoretical Design Division, Los Alamos National Laboratory, Los Alamos, NM, U.S.A. 87545
}

\begin{abstract}
We consider the solution of the fully kinetic (including electrons) Vlasov-Ampère system in a one-dimensional physical space and two-dimensional velocity space (1D-2V) for an arbitrary number of species with a time-implicit Eulerian algorithm. The problem of velocity-space meshing for disparate thermal and bulk velocities is dealt with by an adaptive coordinate transformation of the Vlasov equation for each species, which is then discretized, including the resulting inertial terms. Mass, momentum, and energy are conserved, and Gauss's law is enforced to within the nonlinear convergence tolerance of the iterative solver through a set of nonlinear constraint functions while permitting significant flexibility in choosing discretizations in time, configuration, and velocity space. We mitigate the temporal stiffness introduced by, e.g., the plasma frequency through the use of high-order/low-order (HOLO) acceleration of the iterative implicit solver. We present several numerical results for canonical problems of varying degrees of complexity, including the multiscale ion-acoustic shock wave problem, which demonstrate the efficacy, accuracy, and efficiency of the scheme.
\end{abstract}

Keywords: Conservative discretization, Vlasov-Ampére, adaptive velocity grid, implicit solver, high-order/low-order acceleration, HOLO

\section{Introduction}

In recent years, it has become apparent that kinetic effects (i.e., particle long mean-free-path) can play a significant role in the evolution of high-energy-density (HED) plasma systems, such as inertial confinement fusion (ICF) capsule implosions [1-5]. To study these systems, radiation-hydrodynamic models are typically used; however, to resolve the long mean-free-path effects, it is necessary to employ a kinetic approach. Vlasov-Fokker-Planck codes, such as iFP [6] and FPion [7], have been developed with the goal of resolving ion kinetic effects in weakly collisional regimes with arbitrary Knudsen numbers. However, they continue to treat the electrons as a quasineutral, ambipolar fluid, including only an electron temperature equation. The fluid electron assumption neglects important kinetic plasma effects such as nonlocal electron heat transport, which may be necessary to correctly describe HED plasma phenomena such as shocks and ablation fronts in ICF implosions. This study proposes an efficient and accurate algorithmic solution for simulating the fully kinetic $1 D-2 \mathrm{~V}$ ion-electron system.

There have been attempts to account for nonlocal electron effects within fluid models. A common approach is limiting the electron heat flux to some fraction of the free-streaming flux [8]; another strategy is to spatially convolve the electron heat flux [9]. However, in order to describe electron kinetic effects accurately, it is necessary to solve a fully kinetic model. To this end, the Vlasov-Fokker-Planck(VFP)-Maxwell system of equations may be taken as a first-principles representation of a weakly-coupled plasma. In a one-dimensional spatial system, and assuming an electrostatic field response, this may - without loss of generality - be reduced to a $2 \mathrm{D}$ velocity space described by longitudinal (parallel) and perpendicular velocities. This leads to the 1D-2V VFP-Ampère (or VFP-Poisson) system. The 1D Ampère equation describes the evolution of the longitudinal electric field based on the moments of all species' velocity distribution functions, while the 1D-2V VFP equation describes the evolution of these distribution functions. To simplify the current development and presentation, we explore in this work only the collisionless aspects of the algorithm, i.e., the 1D-2V Vlasov-Ampère system. This in no way compromises our goal of developing a fully kinetic

\footnotetext{
${ }^{*}$ Corresponding author

Email address: andeste@lanl.gov (S. E. Anderson)
} 
simulation tool for plasmas of arbitrary collisionality, as the numerical details of extending the approach described herein to include the Fokker-Planck (FP) collision operator may be considered independently. Interested readers are referred to e.g., Refs. [10-12] for a compatible fully implicit finite-difference approach.

To solve the Vlasov-Ampère system, there are a variety of possible approaches including temporally implicit or explicit applications of particle-in-cell (PIC) methods [13-16], semi-Lagrangian grid-based methods [17--19], or Eulerian grid-based approaches [20-22]. In addition, it is possible to utilize semi-implicit strategies [23], which aim at treating only the stiff physics implicitly, as well as various asymptotic-preserving (AP) schemes [24, 25], which propose discrete formulations capable of capturing the correct asymptotic limit when stiff physics are stepped over. In this work, we apply a temporally fully implicit grid-based Eulerian approach. An implicit approach has significant advantages over explicit schemes, particularly for grid-based approaches where the advective Courant-FriedrichsLewy (CFL) time-step limit is determined by the fastest speed on the velocity grid. With a fully-implicit nonlinear iterative solver, highly temporally multiscale problems - in which the system dynamics are driven on time-scales much longer than the fastest supported time-scales - may become much more tractable. A classic collisionless example is the ion-acoustic shock wave, where the dynamic time-scale is roughly 100 times longer than the inverse electron plasma frequency, and may be 1000 times (or more) longer than the explicit time-scale based on the maximum grid velocity [20]. In addition, a fully implicit iterative scheme exhibits advantages over semi-implicit and AP schemes primarily in the straightforward application of existing strategies to enforce discrete conservation. In the current work, the high-order(HO)/low-order(LO) scheme (HOLO) is used so that the LO (moment) system of equations accelerates convergence of the HO (kinetic) system. The LO system consists of the moments of the plasma species' Vlasov equations coupled to Ampère's equation, while the HO system consists of the Vlasov equations. HOLO approaches have been used to solve a variety of systems [26], from neutron [27] and thermal radiation transport [28] to BGK gas-kinetics [29]. More importantly, the HOLO approach has been applied to the solution of collisionless [13, 20] and collisional [30] plasma systems. In an earlier study by Taitano and Chacón [20], the HOLO approach was used to accelerate Vlasov-Ampère convergence by using the LO system to efficiently evaluate the electric field with the higher-order moment closure provided by the HO system. In this work, we generalize this study both by applying a non-centered time integration scheme and by considering the adaptive velocity-space strategy proposed in Ref. [11].

When solving the Vlasov-Ampère system, a static velocity mesh may become inefficient in problems where the species temperatures and bulk velocities exhibit significant temporal and spatial variations. Specifically, the mesh must be large enough to capture both the shift in the bulk velocity and temperature evolution at the hottest location in space and time (the largest thermal speed), while maintaining a sufficient resolution for the coldest location (the smallest thermal speed) for all species. In contrast, a mesh that dynamically expands/contracts in space and time while shifting the center to track changes in their bulk velocities may efficiently resolve the hottest/coldest regions of each species. The present work applies an analytic transformation to the Vlasov equation for each species $\alpha$, scaling it by a normalizing speed $v_{\alpha}^{*}$ (which is a function of the thermal speed $v_{t h, \alpha}$ ) and shifting the velocity space by an offset velocity $u_{\|, \alpha}^{*}$ (which is a function of the bulk velocity $u_{\|, \alpha}$ ). This is similar to the approach described in Refs. [6, 7, 25, 31, 32].

To preserve the numerical accuracy of long-time simulations, we desire a discretization scheme for which the continuum symmetries of the governing equations (leading to mass, momentum, and energy conservation) are preserved in the discrete. Without a discrete conservation principle, long-term simulations may produce significant violations of the conservation properties due to accumulated discretization errors, which can manifest as numerical plasma heating or cooling [30], or a departure of the solution from the asymptotic hydrodynamic manifold. Indeed, as we shall demonstrate later, the failure to ensure discrete charge conservation (i.e., enforcing the discrete Gauss's law) leads to catastrophic failure in simulations, with significant departure from the correct solution. Further, in the case of the Vlasov-Fokker-Planck equation, Taitano et al. [6] showed that even neglecting to ensure discrete momentum and energy conservation relationships only in the Vlasov equation (while enforcing it in the Fokker-Planck collision operator) leads to extremely large numerical errors $[\sim O(1)]$. Thus, discrete conservation is key for achieving high fidelity and accuracy.

Broadly, we will distinguish between two different strategies for achieving discrete conservation. The first, which we term a "passive" approach, relies on specifically chosen discretizations that "passively" preserve the structure of the governing equations. This is a general catch-all for the symplectic and Hamiltonian-preserving techniques for plasma physics systems described by Morrison [33]. Such techniques have also been called "structure-preserving" [33, 34], and are in general very effective at preserving invariants - for the Vlasov-Maxwell system, Shiroto et al. [34] demonstrated conservation errors on the order of machine precision. However, from our perspective, there are two significant shortcomings of this approach. The first is that they are generally only possible through central differencing 
schemes, which are not monotonic, positivity-preserving, or non-oscillatory (all of which are desirable properties). The second is that, in the case of the velocity-space transformed Vlasov equation used in this work, it is not readily apparent whether such "structure-preserving" discretizations are possible. The second strategy, which we term an "active" approach, is the strategy we employ in this work to achieve discrete conservation. To implement this approach, we introduce Lagrange-multiplier-like constraint functions into the discretized governing equations. These "nonlinear constraint functions" are defined so as to actively enforce certain continuum symmetries of the governing equations, ensuring conservation of e.g., mass, momentum, and energy in the discretized system. The primary benefit of the "active" strategy is that it permits a choice of arbitrary discretization schemes in both time (e.g., backward Euler, or BDF2) and phase space (e.g., SMART [35] or WENO [36]).

The present approach is similar to the strategies used in Refs. [20] and [31]; however, there are some important differences. In the previous Vlasov-Ampère implementation, the approach relied on a time-centered Crank-Nicolson integrator to achieve energy conservation. The current work uses a BDF2 temporal integration scheme (which is more appropriate for an eventual application to the collisional system), and can in principle be applied to an arbitrary temporal integration scheme. Further, the approach for enforcing discrete conservation with the velocity-space adaptivity (as in Ref. [31] ) must be modified because of interaction with the additional constraint functions. Thus, the current work delivers an implicit algorithm for the fully kinetic, arbitrary-species 1D-2V Vlasov-Ampère system, which conserves mass, momentum, and energy to within nonlinear convergence tolerance. The algorithm is adaptive in the velocity space to ease meshing requirements due to temporal and spatial variations in the local bulk velocity and thermal speed of each species, while the nonlinear constraint functions that ensure conservation also allow substantial freedom of choice for temporal and advective discretizations.

The rest of this paper is organized as follows. Section 2 gives an overview of the governing equations for the Vlasov-Ampère/Poisson system in $1 \mathrm{D}-2 \mathrm{~V}$, its transformation in the velocity space, and the continuum-conservation symmetries of the system. Section 3 describes the discretization of the Vlasov system. Section 4 provides details of our strategy for ensuring the continuum conservation symmetries in the discretized system. In Sec. 5 we present our nonlinear iterative strategy for solving the discretized system implicitly in time using a HOLO acceleration scheme. We present numerical results highlighting the accuracy and performance of the algorithm for several canonical problems of varying difficulty in Sec. 6 and provide concluding remarks in Sec.7.

\section{Vlasov-Ampère system of equations}

The Vlasov-Ampère/Poisson system may be regarded as a first-principles representation for a fully ionized electrostatic collisionless plasma. The governing equations are the Vlasov equations for each species $\alpha$,

$$
\partial_{t} f_{\alpha}+\nabla_{\boldsymbol{x}} \cdot\left(\boldsymbol{v} f_{\alpha}\right)+\frac{q_{\alpha}}{m_{\alpha}} \boldsymbol{E} \cdot \boldsymbol{\nabla}_{\boldsymbol{v}}\left(f_{\alpha}\right)=0
$$

which describe the evolution in phase space of distribution functions, $f_{\alpha}$, and Ampère's equation,

$$
\epsilon_{0} \partial_{t} \boldsymbol{E}+\sum_{\alpha} q_{\alpha} \boldsymbol{\Gamma}_{\alpha}=0
$$

which describes the evolution of the electric field, $\boldsymbol{E}$. In Eqs. (1) and (2), $\boldsymbol{v}$ is the particle velocity, and $q_{\alpha}$ and $m_{\alpha}$ are the particle charge and mass of species $\alpha$, respectively. We define the particle flux density to be $\boldsymbol{\Gamma}_{\alpha} \equiv n_{\alpha} \boldsymbol{u}_{\alpha}$, where $n_{\alpha}$ is the number density and $\boldsymbol{u}_{\alpha}$ the bulk velocity. The Vlasov-Poisson system instead utilizes Gauss's law,

$$
\epsilon_{0} \boldsymbol{\nabla}_{\boldsymbol{x}} \cdot \boldsymbol{E}-\sum_{\alpha} q_{\alpha} n_{\alpha}=0
$$

and the electric potential, $\Phi$, defined by $\nabla_{\boldsymbol{x}} \Phi=-\boldsymbol{E}$, to obtain Poisson's equation,

$$
\epsilon_{0} \nabla_{\boldsymbol{x}}^{2} \Phi+\sum_{\alpha} q_{\alpha} n_{\alpha}=0
$$

The Vlasov-Poisson and Vlasov-Ampère systems can be shown to be equivalent through charge conservation, i.e.,

$$
0=\boldsymbol{\nabla}_{\boldsymbol{x}} \cdot\left[\epsilon_{0} \partial_{t} \boldsymbol{E}+\sum_{\alpha} q_{\alpha} \boldsymbol{\Gamma}_{\alpha}\right]=\partial_{t}\left[\epsilon_{0} \boldsymbol{\nabla}_{\boldsymbol{x}} \cdot \boldsymbol{E}-\sum_{\alpha} q_{\alpha} n_{\alpha}\right] \Longrightarrow \partial_{t} \sum_{\alpha} q_{\alpha} n_{\alpha}+\boldsymbol{\nabla}_{\boldsymbol{x}} \cdot \sum_{\alpha} q_{\alpha} \boldsymbol{\Gamma}_{\alpha}=0
$$


In one-dimensional configuration space, Eqs. (1)-2) may be expressed as

$$
\begin{array}{r}
\partial_{t} f_{\alpha}+\partial_{x}\left(v_{\|} f_{\alpha}\right)+\frac{q_{\alpha}}{m_{\alpha}} E_{\|} \partial_{v_{\|}}\left(f_{\alpha}\right)=0, \\
\epsilon_{0} \partial_{t} E_{\|}+\sum_{\alpha} q_{\alpha} \Gamma_{\|, \alpha}=\bar{j}_{\|},
\end{array}
$$

where

$$
\bar{\Phi}=\frac{1}{x_{\max }-x_{\min }} \int_{x_{\min }}^{x_{\max }} \Phi d x
$$

denotes the spatial average of a quantity $\Phi$. We note that we have included the spatial average of the current density $\bar{j}_{\|}$ in Ampère's equation. This is necessary in 1D periodic systems to preserve Galilean invariance, and to ensure $\bar{E}_{\|}=0$ [13-15]. For details, see Appendix A Without loss of generality, the velocity-space domain may be reduced to two dimensions by invoking cylindrical symmetry, such that the velocity coordinates reduce from $\left(v_{x}, v_{y}, v_{z}\right)$ to $\left(v_{\|}, v_{\perp}\right)$. In Eqs. (6)-(7), we use the parallel notation to indicate vector components.

\subsection{Velocity-space coordinate transformation}

We perform a phase-space coordinate transformation of Eq. (6) as proposed in Refs. [25, 31, 32]. Namely, for each species $\alpha$ we transform the velocity space (i.e. the velocity coordinate $v$ ) by a translating with a reference offset velocity $u_{\|, \alpha}^{*}(x, t)$ and then normalizing by a reference speed $v_{\alpha}^{*}(x, t)$. These quantities are related to each species bulk-flow velocity and thermal speed, respectively, but are not necessarily equal to them. For each species, we thus define a transformed velocity coordinate $c$ as

$$
\boldsymbol{c}=\hat{\boldsymbol{v}}-\hat{u}_{\|, \alpha}^{*} \boldsymbol{e}_{\|}
$$

where $\hat{\boldsymbol{v}}=\boldsymbol{v} / v_{\alpha}^{*}(x, t)$ is the normalized velocity coordinate, $\hat{u}_{\|, \alpha}^{*}=u_{\|, \alpha}^{*} / v_{\alpha}^{*}$ is the normalized offset velocity, and $\boldsymbol{e}_{\|}$ is the unit vector along $x$. The velocity coordinate $v$ may be thus decomposed using $c, v_{\alpha}^{*}, u_{\|, \alpha}^{*}$, and $\boldsymbol{e}_{\|}$as,

$$
\boldsymbol{v}=v_{\alpha}^{*}(x, t) \boldsymbol{c}+u_{\|, \alpha}^{*}(x, t) \boldsymbol{e}_{\|}
$$

In this work, the goal of the velocity transformation is to ensure that the computational velocity-space domain (i.e. the set of logical velocity space coordinates) is identical for all species. Thus, regardless of spatio-temporal variations in bulk velocity and temperature (thermal speed) between species, we are able to use the same mesh for all species in velocity space.

For full details of the transformation, we refer readers to the work of Taitano et al. [31]. The final form of the transformed Vlasov equation is thus

$$
\begin{aligned}
\partial_{t} \tilde{f}_{\alpha}+\partial_{x}\left(v_{\alpha}^{*} \hat{v}_{\|} \tilde{f}_{\alpha}\right)+\frac{q_{\alpha}}{m_{\alpha} v_{\alpha}^{*}} E_{\|} \partial_{c_{\|}} \tilde{f}_{\alpha} & \\
& -\frac{1}{v_{\alpha}^{*}} \nabla_{\boldsymbol{c}} \cdot\left\{\left[\partial_{t}\left(\boldsymbol{c} v_{\alpha}^{*}+\boldsymbol{e}_{\|} u_{\|, \alpha}^{*}\right)+\partial_{x}\left(\boldsymbol{c} v_{\alpha}^{*}+\boldsymbol{e}_{\|} u_{\|, \alpha}^{*}\right) \hat{v}_{\|} v_{\alpha}^{*}\right] \tilde{f}_{\alpha}\right\}=0,
\end{aligned}
$$

where $\tilde{f}_{\alpha}=f_{\alpha}\left(v_{\alpha}^{*}\right)^{3}$. In what follows, we will use the shorthand notation

$$
\left\langle\Phi\left(c_{\|}, c_{\perp}\right), \tilde{f}_{\alpha}\left(c_{\|}, c_{\perp}\right)\right\rangle_{c} \equiv 2 \pi \int_{-\infty}^{\infty} d c_{\|} \int_{0}^{\infty} c_{\perp} d c_{\perp} \Phi\left(c_{\|}, c_{\perp}\right) \tilde{f}_{\alpha}\left(c_{\|}, c_{\perp}\right)
$$

to denote the velocity-space moment of a function, $\tilde{f}_{\alpha}\left(c_{\|}, c_{\perp}\right)$, with the weight, $\Phi\left(c_{\|}, c_{\perp}\right)$.

\subsection{Summary of key continuum symmetries}

Equations (7) and (10) conserve mass, momentum, and energy in the continuum. However, these continuum properties are not automatically preserved when the governing equations are discretized, as we shall see in Sec. 4. In what follows, we will highlight particular symmetries of the governing equations that lead to the desired conservation properties. Detailed proofs of the conservation properties including these symmetries can be found in Appendix B 


\subsubsection{Symmetries relating to the Vlasov equation velocity-space transformation}

For the transformed Vlasov equation, we recall that our independent velocity variables have become $\left(c_{\|}, c_{\perp}\right)$. However, momentum and energy conservation are still defined in terms of $v_{\|}$and $v^{2}$ (the original velocity) moments of $f_{\alpha}$. The important point is that $\boldsymbol{v}$ moments do not commute with temporal and spatial derivatives in the transformed space, e.g.,

$$
\begin{aligned}
\left\langle m_{\alpha} v_{\|}, \partial_{t} \tilde{f}_{\alpha}\right\rangle_{\boldsymbol{c}} & \neq\left\langle 1, \partial_{t}\left(m_{\alpha} v_{\|} \tilde{f}_{\alpha}\right)\right\rangle_{\boldsymbol{c}}, \\
\left\langle m_{\alpha} v_{\|}, \partial_{x}\left(v_{\|} \tilde{f}_{\alpha}\right)\right\rangle_{\boldsymbol{c}} & \neq\left\langle 1, \partial_{x}\left(m_{\alpha} v_{\|}^{2} \tilde{f}_{\alpha}\right)\right\rangle_{\boldsymbol{c}} .
\end{aligned}
$$

Thus, to obtain the momentum conservation theorem for the transformed system, we must utilize integration by parts and the product rule on the temporal and spatial components of the Vlasov equation to obtain the identity:

$$
\begin{aligned}
\left\langle m_{\alpha} v_{\|}, \partial_{t} \tilde{f}_{\alpha}\right\rangle_{\boldsymbol{c}}+\left\langle m_{\alpha} v_{\|}, \partial_{x}\left(v_{\|} \tilde{f}_{\alpha}\right)\right\rangle_{\boldsymbol{c}}-\frac{1}{v_{\alpha}^{*}}\left\langle m_{\alpha} v_{\|}, \nabla_{\boldsymbol{c}} \cdot\left\{\left[\partial_{t}(\boldsymbol{v})+\partial_{x}(\boldsymbol{v}) \hat{v}_{\|} v_{\alpha}^{*}\right] \tilde{f}_{\alpha}\right\}\right\rangle_{\boldsymbol{c}} \\
=\left\langle 1, \partial_{t}\left(m_{\alpha} v_{\|} \tilde{f}_{\alpha}\right)\right\rangle_{\boldsymbol{c}}+\left\langle 1, \partial_{x}\left(m_{\alpha} v_{\|}^{2} \tilde{f}_{\alpha}\right)\right\rangle_{\boldsymbol{c}},
\end{aligned}
$$

which must be satisfied locally for each species. Likewise, for the energy conservation theorem we must have the identity:

$$
\begin{aligned}
\left\langle m_{\alpha} \frac{1}{2} \boldsymbol{v}^{2}, \partial_{t} \tilde{f}_{\alpha}\right\rangle_{\boldsymbol{c}}+\left\langle m_{\alpha} \frac{1}{2} \boldsymbol{v}^{2}, \partial_{x}\left(v_{\|} \tilde{f}_{\alpha}\right)\right\rangle_{\boldsymbol{c}} & -\frac{1}{v_{\alpha}^{*}}\left\langle m_{\alpha} \frac{1}{2} \boldsymbol{v}^{2}, \nabla_{\boldsymbol{c}} \cdot\left\{\left[\partial_{t}(\boldsymbol{v})+\partial_{x}(\boldsymbol{v}) v_{\|}\right] \tilde{f}_{\alpha}\right\}\right\rangle_{\boldsymbol{c}} \\
& =\left\langle 1, \partial_{t}\left(m_{\alpha} \frac{1}{2} \boldsymbol{v}^{2} \tilde{f}_{\alpha}\right)\right\rangle_{\boldsymbol{c}}+\left\langle 1, \partial_{x}\left(m_{\alpha} \frac{1}{2} \boldsymbol{v}^{2} v_{\|} \tilde{f}_{\alpha}\right)\right\rangle_{\boldsymbol{c}} .
\end{aligned}
$$

For an arbitrary discretization, these identities will generally not be satisfied simultaneously or even independently.

\subsubsection{Symmetries relating to Ampère's equation}

In addition to the preceding symmetries for the velocity-space adaptivity, there are several which must be satisfied for the Vlasov-Ampère system as a whole. The first is the equivalence through charge conservation between Gauss's law, Ampère's equation, and the continuity equation:

$$
\partial_{t} \rho_{q}+\partial_{x} j_{\|}=\sum_{\alpha} q_{\alpha}\left[\left\langle 1, \partial_{t} \tilde{f}_{\alpha}\right\rangle_{c}+\left\langle 1, \partial_{x}\left(v_{\|} \tilde{f}_{\alpha}\right)\right\rangle_{c}\right] .
$$

In Eq. 16 , we defined $\rho_{q} \equiv \sum_{\alpha} q_{\alpha} n_{\alpha}$ and $j_{\|} \equiv \sum_{\alpha} q_{\alpha} \Gamma_{\|, \alpha}$, and we further define $\left\langle 1, \tilde{f}_{\alpha}\right\rangle_{c}=n_{\alpha}$ and $\left\langle v_{\|}, \tilde{f}_{\alpha}\right\rangle_{c}=$ $\Gamma_{\|, \alpha}$. We see that the charge density in Gauss's law and the current in Ampère's equation must be proportional to the particle number density and flux in the continuity equation [i.e., the $\boldsymbol{v}^{0}$ moment of the transformed Vlasov equation, Eq. [10p]. As we will see shortly, the crux is that the current in Ampère's equation (which drives the electric field $E_{\|}$) must be consistent with Eq. (16, thereby ensuring that $\epsilon_{0} \partial_{x} E_{\|}=\rho_{q}$ discretely. This equivalence is in some sense a fundamental "zeroth-order" requirement for the Vlasov-Ampère system, and, as we shall see shortly, neglecting it in the discrete will lead to catastrophic errors [37-40].

The second requirement is that the $m_{\alpha} v_{\|}$moment of the acceleration operator in Eq. 10 pust produce a number density $n_{\alpha}$ that is identical to the one in Gauss's law. If we sum the moment of this term over all species we find

$$
\sum_{\alpha} \frac{q_{\alpha}}{m_{\alpha} v_{\alpha}^{*}} E_{\|}\left\langle m_{\alpha} v_{\|}, \partial_{c_{\|}} \tilde{f}_{\alpha}\right\rangle_{c}=-E_{\|} \sum_{\alpha} q_{\alpha} n_{\alpha}=-\partial_{x}\left(\epsilon_{0} \frac{1}{2} E_{\|}^{2}\right)
$$

We see that this symmetry introduces the divergence of the electrostatic energy into the momentum equation, which is key to achieve momentum conservation.

To arrive at energy conservation in the Vlasov-Ampère system, we again inspect the acceleration operator. Taking the $m_{\alpha} \frac{1}{2} \boldsymbol{v}^{2}$ moment of the acceleration operator we find:

$$
\sum_{\alpha} \frac{q_{\alpha}}{m_{\alpha} v_{\alpha}^{*}} E_{\|}\left\langle m_{\alpha} \frac{1}{2} v^{2}, \partial_{c_{\|}} \tilde{f}_{\alpha}\right\rangle_{\boldsymbol{c}}=-E_{\|} \sum_{\alpha} q_{\alpha} \Gamma_{\|, \alpha}=E_{\|}\left[\epsilon_{0} \partial_{t}\left(E_{\|}\right)-\bar{j}_{\|}\right]=\partial_{t}\left(\epsilon_{0} \frac{1}{2} E_{\|}^{2}\right)-E_{\|} \bar{j}_{\|} .
$$


Thus, we see that this moment must produce a particle flux density that is consistent with the current that appears in Ampère's equation. Further, we observe that, while the equivalence

$$
E_{\|} \partial_{t} E_{\|}=\partial_{t}\left(\frac{1}{2} E_{\|}^{2}\right)
$$

is true in the continuum, it will not be so for an arbitrary temporal discretization.

\subsection{Strategy for enforcing continuum symmetries in the discrete}

To enforce the preceding continuum symmetries in the discretized Vlasov-Ampère system, we introduce a set of nonlinear constraint functions to the discrete representation of Eq. (10). These added nonlinear constraints take the form of Lagrange-multiplier-like coefficients and associated operators. For the symmetries related to the velocity-space adaptivity, we introduce the constraint functions $\gamma_{t}$ and $\gamma_{x}$, which modify the discretized inertial terms in a manner similar to that of in Refs. [2, 11]. In the discretized form of Eq. [10], these will be highlighted in red. Following a similar approach to Refs. [20, 30] for the Vlasov-Ampère symmetries, we introduce the constraint functions $\xi, \phi$, and $\gamma_{q}$, which appear in their own phase-space advection "pseudo-operators". We will depict these highlighted in blue. These constraint functions all act to expose the underlying continuum symmetries of the governing equations and eliminate the truncation errors between different discrete operators, which would break the symmetries. We note that, while having five distinct nonlinear constraint functions $\left(\xi, \phi, \gamma_{q}, \gamma_{t}\right.$, and $\left.\gamma_{x}\right)$ to enforce only three conservation laws (charge, momentum, and energy conservation) may seem overconstrained, this is not the case. The critical distinction is that we are not directly enforcing the conservation laws themselves, but rather the symmetries that lead to those laws. Details on the formulation of these nonlinear constraints are discussed in Secs. 3 , 4 .

\section{Numerical implementation}

\subsection{Discretization of the transformed Vlasov equation}

The Vlasov equation, Eq. (10), is discretized using finite differences in the transformed phase space as follows. The discrete cylindrical cell volume in the velocity space for a uniform velocity mesh is

$$
\tilde{\Omega}_{j, k} \equiv 2 \pi c_{\perp, k} \Delta c_{\perp} \Delta c_{\|},
$$

while the total discrete volume including the configuration space on a uniform mesh is

$$
\Delta \tilde{V}_{i, j, k}=\Delta x \tilde{\Omega}_{j, k} .
$$

The quantities $\Delta c_{\|}, \Delta c_{\perp}$, and $\Delta x$ are the mesh spacings for the parallel velocity, perpendicular velocity, and configuration space, respectively. The domains are defined to be

$$
x \in\left[x_{\min }, x_{\max }\right], v_{\|} \in\left[v_{\|, \min }, v_{\|, \max }\right], v_{\perp} \in\left[0, v_{\perp, \max }\right],
$$

such that

$$
L_{x} \equiv x_{\text {max }}-x_{\text {min }}, L_{\|} \equiv v_{\|, \max }-v_{\|, \min }, L_{\perp} \equiv v_{\perp, \max } .
$$

Thus, for the transformed velocity space the domain becomes

$$
c_{\|} \in\left[c_{\|, \min }, c_{\|, \max }\right], c_{\perp} \in\left[0, c_{\perp, \max }\right],
$$

with

$$
\tilde{L}_{\|}=\frac{L_{\|}}{v_{\alpha}^{*}}=c_{\|, \max }-c_{\|, \min }, \tilde{L}_{\perp}=\frac{L_{\perp}}{v_{\alpha}^{*}}=c_{\perp, \max } .
$$

The mesh spacings $\Delta x, \Delta c_{\|}, \Delta c_{\perp}$ are given by

$$
\Delta x=\frac{L_{x}}{N_{x}}, \Delta c_{\|}=\frac{\tilde{L}_{\|}}{N_{\|}}, \Delta c_{\perp}=\frac{\tilde{L}_{\perp}}{N_{\perp}} .
$$

Here, $N_{x}, N_{\|}$, and $N_{\perp}$ are the numbers of cells along each coordinate. The coordinates $i, j, k$ are defined to be at the cell centers, so that the boundary of the domain in each direction is on cell faces. Thus, a cell-center quantity 
$\Phi_{j}$ spans $j \in\left[1, N_{\|}\right]$, while a cell-face quantity $\Phi_{j+\frac{1}{2}}$ spans $j \in\left[0, N_{\|}\right]$. Here, we reiterate that the transformed velocity-space domain $c_{\|} \in\left[c_{\|, \min }, c_{\|, \max }\right], c_{\perp} \in\left[0, c_{\perp, \max }\right]$ (the computational velocity-space domain) is the same for all species, and is constant in space and time. The spatio-temporal variations in bulk velocity and thermal speed (temperature) between species are dealt with through the reference speed $v_{\alpha}^{*}$ and offset velocity $u_{\|, \alpha}^{*}$.

Discrete moments in the velocity space are computed via a midpoint quadrature as

$$
\langle A, B\rangle_{\delta \boldsymbol{c}} \approx \sum_{j=1}^{N_{\|}} \sum_{k=1}^{N_{\perp}} \tilde{\Omega}_{j, k} A_{j, k} B_{j, k}
$$

for scalars defined at the cell centers, and as

$$
\langle 1, \boldsymbol{A} \cdot \boldsymbol{B}\rangle_{\delta \boldsymbol{c}} \approx \sum_{j=0}^{N_{\|}} \sum_{k=1}^{N_{\perp}} \tilde{\Omega}_{j+\frac{1}{2}, k} A_{\|, j+\frac{1}{2}, k} B_{\|, j+\frac{1}{2}, k}+\sum_{j=1}^{N_{\|}} \sum_{k=0}^{N_{\perp}} \tilde{\Omega}_{j, k+\frac{1}{2}} A_{\perp, j, k+\frac{1}{2}} B_{\perp, j, k+\frac{1}{2}}
$$

for scalar products of velocity-space vectors defined at the cell faces. Quantities at half-indices (e.g., $\left.j+\frac{1}{2}\right)$ are at the cell faces.

A discretization of the Vlasov equation, Eq. [10, which includes all the relevant 'pseudo-operators' and nonlinear constraint functions to enforce discrete conservation, is then given by

$$
\begin{aligned}
& \delta_{t} \tilde{f}_{\alpha, i, j, k}^{p+1}+\underbrace{\delta_{x}\left[v_{\|, \alpha, j}^{p} \overline{\left(\tilde{f}_{\alpha}^{p+1}\right)_{j, k}^{v_{\|}}}\right]_{i}}+\underbrace{\frac{q_{\alpha}}{m_{\alpha}} \frac{E_{\|, i}^{p+1}}{v_{\alpha, i}^{*, p}} \delta_{c_{\|}}\left[\overline{\left.\left(\tilde{f}_{\alpha}^{p+1}\right)_{i, k}^{q_{\alpha} E_{\|}}\right]_{j}}\right.} \\
& \text { (a) } \underbrace{}_{(b)}
\end{aligned}
$$

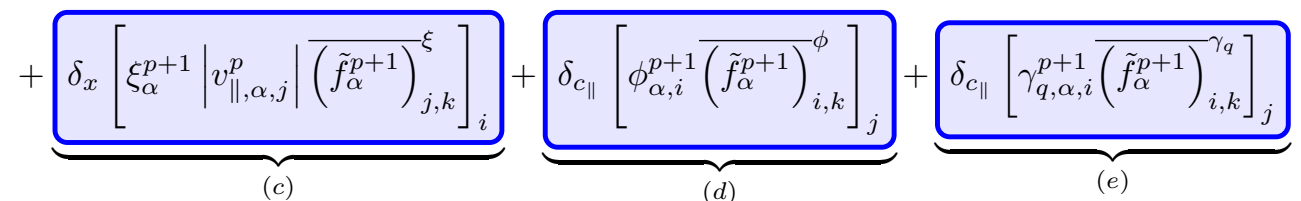

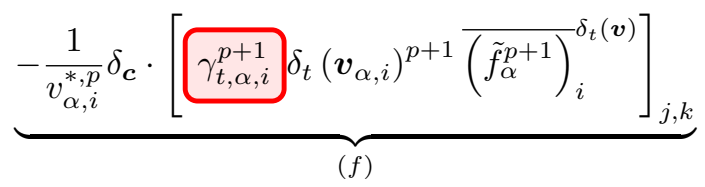

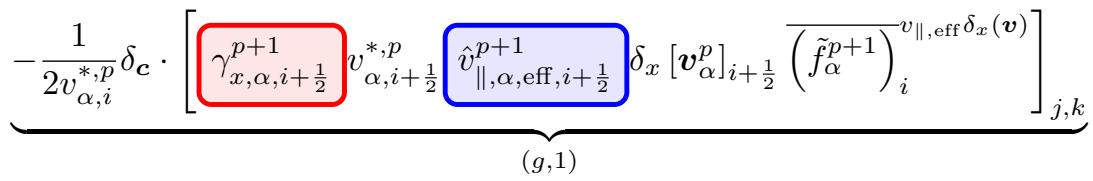

$$
\begin{aligned}
& \underbrace{-\frac{1}{2 v_{\alpha, i}^{*, p}} \delta_{\boldsymbol{c}} \cdot[\underbrace{}_{\gamma_{x, \alpha, i-\frac{1}{2}}^{p+1}} v_{\alpha, i-\frac{1}{2}}^{*, p} \underbrace{p+1}_{\|, \alpha, \text { eff }, i-\frac{1}{2}} \delta_{x}\left[\boldsymbol{v}_{\alpha}^{p}\right]_{i-\frac{1}{2}} \overline{\left(\tilde{f}_{\alpha}^{p+1}\right)_{i}} v_{\|, \text {eff }} \delta_{x}(\boldsymbol{v})]_{j, k}}_{(g, 2)}=0 .
\end{aligned}
$$

Here, we define the following notation

$$
\begin{aligned}
\delta_{t}(F)^{p+1} & \equiv \frac{b^{p+1} F^{p+1}+b^{p} F^{p}+b^{p-1} F^{p-1}}{\Delta t^{p}}, \\
\delta_{x} F_{i} & \equiv \frac{F_{i+\frac{1}{2}}-F_{i-\frac{1}{2}}}{\Delta x}, \\
\delta_{c_{\|}} F_{\|, j, k} & \equiv \frac{F_{\|, j+\frac{1}{2}, k}-F_{\|, j-\frac{1}{2}, k}}{\Delta c_{\|}}, \\
\delta_{c_{\perp}} F_{\perp, j, k} & \equiv \frac{c_{\perp, k+\frac{1}{2}} F_{\perp, j, k+\frac{1}{2}}-c_{\perp, k-\frac{1}{2}} F_{\perp, j, k-\frac{1}{2}}}{c_{\perp, k} \Delta c_{\perp}}, \\
\delta_{\boldsymbol{c}} \cdot[\boldsymbol{F}]_{j, k} & \equiv \delta_{c_{\|}} F_{\|, j, k}+\delta_{c_{\perp}} F_{\perp, j, k},
\end{aligned}
$$




$$
\hat{v}_{\|, \alpha, \mathrm{eff}, i+\frac{1}{2}, j}^{p+1} \equiv\left(c_{\|, j}+\hat{u}_{\|, \alpha, i+\frac{1}{2}}^{*, p}+\hat{\xi}_{\alpha, i+\frac{1}{2}}^{p+1}\left|c_{\|, j}+\hat{u}_{\|, \alpha, i+\frac{1}{2}}^{*, p}\right|\right),
$$

with $v_{\alpha, i+\frac{1}{2}}^{*, p}=\frac{v_{\alpha, i}^{*, p}+v_{\alpha, i+1}^{*, p}}{2}, \hat{u}_{\|, \alpha, i+\frac{1}{2}}^{*, p}=\frac{\hat{u}_{\|, \alpha, i}^{*, p}+\hat{u}_{\|, \alpha, i+1}^{*, p}}{2}$, and $\boldsymbol{v}_{\alpha, i, j, k}^{p}=v_{\alpha, i}^{*, p} \boldsymbol{c}_{j, k}+u_{\|, \alpha, i}^{*, p} \boldsymbol{e}_{\|}$. Note that the nonlinear constraint function $\xi$ is included in the definition of $\hat{v}_{\|, \alpha, \text { eff }, i-\frac{1}{2}}^{p+1}$ (boxed in blue). In Eq. 23, we have utilized a secondorder backwards finite difference scheme in time (BDF2, [41, Chapter III]), with coefficients $b^{p+1}=1.5, b^{p}=-2$, and $b^{p-1}=0.5$. The temporal index is $p$. Here, as in Refs. [11, 31], we lag the time-level of the reference speed $v_{\alpha, i}^{*, p}$ and offset velocity $\hat{u}_{\|, \alpha, i}^{*, p}$ for robustness; thus, for discrete temporal derivatives involving these quantities, we have

$$
\begin{gathered}
\delta_{t}\left(v_{\alpha}^{*} F\right)^{p+1}=\frac{b^{p+1} v_{\alpha}^{*, p} F^{p+1}+b^{p} v_{\alpha}^{*, p-1} F^{p}+b^{p-1} v_{\alpha}^{*, p-2} F^{p-1}}{\Delta t^{p}} \\
\delta_{t}\left(\hat{u}_{\|, \alpha}^{*} F\right)^{p+1}=\frac{b^{p+1} \hat{u}_{\|, \alpha}^{*, p} F^{p+1}+b^{p} \hat{u}_{\|, \alpha}^{*, p-1} F^{p}+b^{p-1} \hat{u}_{\|, \alpha}^{*, p-2} F^{p-1}}{\Delta t^{p}} .
\end{gathered}
$$

For compactness of notation, we define an advective interpolation operator acting on a scalar $\phi$ at a cell face based on an advection coefficient $a$ :

$$
\overline{(\Phi)}_{\text {face }}^{a}=\sum_{i^{\prime}=1}^{N} \omega_{\text {face }, i^{\prime}}(a, \phi) \phi_{i^{\prime}}
$$

In Eq. 32, $\omega_{\text {face, } i^{\prime}}$ are interpolation weights for the $i^{\prime}$ cells surrounding the cell face, and $\phi_{i^{\prime}}$ are the values of the interpolated quantity in those cells. We note here that the cell-center electric field is defined as the interpolation of adjacent cell-face electric fields,

$$
E_{\|, i}^{p+1}=\frac{E_{\|, i+\frac{1}{2}}^{p+1}+E_{\|, i-\frac{1}{2}}^{p+1}}{2} .
$$

In Eq. 23 , terms $(a)$ and $(b)$ represent the discrete form of the physical configuration-space advection and velocityspace advection due to the acceleration of the electric field, respectively. Terms $(c),(d)$, and $(e)$ (boxed in blue) are the discretized forms of the 'pseudo-operators' arising from the nonlinear constraint coefficients, $\xi, \phi$, and $\gamma_{q}$. These constraint functions are responsible for enforcing the symmetries discussed in Sec.2.2.2 relating to the Vlasov-Ampère coupling in the discretized system. Terms $(f)$ and $(g)$ are the discretized versions of the inertial terms arising from the respective temporal and spatial gradients in $v_{\alpha}^{*}$ and $\hat{u}_{\|, \alpha}^{*}$, which also include two nonlinear constraint functions, $\gamma_{t}$ and $\gamma_{x}$, (boxed in red). The constraint functions $\gamma_{t}$ and $\gamma_{x}$ are responsible for enforcing the conservation symmetries in Sec. 2.2.1 relating to the velocity-space transformation. Note in the velocity-space inertial terms pertaining to $\gamma_{x}$, the term $\hat{v}_{\|, \alpha, \mathrm{eff}, i-\frac{1}{2}}^{p+1}$ is boxed in blue to indicate that it also contains the constraint function $\xi$. A discussion of the function and definition of the constraint functions and their 'pseudo-operators' is presented in Sec. 4, with more detailed derivations left to Appendix D and Appendix E.

In the present study, several different advective schemes have been utilized for different terms, in accordance with an empirical hierarchy of priority based on observed sensitivity and behavior of different terms. In general, we have observed that the electrons are highly sensitive to numerical dissipation, particularly with schemes (such as SMART [35]) that switch between low-order (upwinding) and higher-order schemes (e.g., QUICK [42]). Thus, for the electron physical configuration space advection [term $(a)$ ], we have chosen a relatively low-dissipation 5th-order WENO scheme (WENO5 [36]). While it does not possess the positivity- and monotonicity-preserving properties of schemes such as SMART, WENO5 is more robust than a central differencing scheme. The electron physical velocity space advection term [term $(b)$ ] is more sensitive still to numerical dissipation, and so, while it is less robust overall than WENO5, a central-differencing scheme is used. The ions are not as sensitive to dissipation, so the SMART scheme is used for the configuration-space advection. This is because of its monotonicity- and positivity-preserving properties, as well as for being well-posed for nonlinear iterative methods. It is also cheaper to evaluate than the WENO5 scheme. In the ion electrostatic acceleration operator, we use WENO5 for increased robustness relative to a central-differencing scheme. For the velocity-space adaptivity inertial terms $(f)$ and $(g)$, we use WENO5 for all species for low dissipation and greater robustness over central differencing. The choice of discretization for the 'pseudo-operators' $(c),(d)$, and $(e)$ is generally much less restrictive - as we shall see, these terms do not affect the order of accuracy of the scheme. Thus, for the charge-conservation pseudo-operator $(c)$, we use a straightforward upwind discretization for simplicity and robustness. For the momentum- and energy-conservation pseudo-operators $(d)$ and $(e)$, we use central differencing primarily for simplicity and to minimize dissipation in velocity space. 


\subsection{Discretization of Ampère's equation, Eq. (7)}

We follow the approach of Ref. [20] and define the electric field $E_{\|, i+\frac{1}{2}}^{p}$ at cell-faces. The discrete Ampère equation for the cell-face electric field is thus

$$
\epsilon_{0} \delta_{t} E_{\|, i+\frac{1}{2}}^{p+1}+\sum_{\alpha}^{N_{s p}} q_{\alpha} \widehat{\Gamma}_{\|, \alpha, i+\frac{1}{2}}^{p+1}=\bar{j}_{\|}^{p+1} .
$$

The cell-face particle flux density must be defined to preserve energy conservation. Thus, instead of being defined based on the "true" momentum moment of $\tilde{f}_{\alpha}$,

$$
\Gamma_{\|, \alpha, i}^{p+1}=\left\langle v_{\|}, \tilde{f}_{\alpha, i, j, k}^{p+1}\right\rangle_{\delta c}
$$

$\widehat{\Gamma}_{\|, \alpha, i+\frac{1}{2}}^{p+1}$ is defined from the $\frac{1}{2} m_{\alpha} \boldsymbol{v}^{2}$ moment of the electrostatic acceleration operator (see the discussion of Eq. 18 , in Sec.2.2.2:

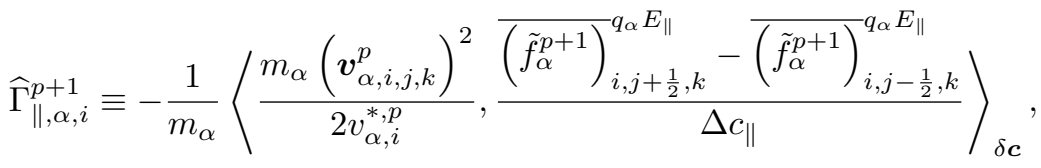

$$
\begin{aligned}
& \widehat{\Gamma}_{\|, \alpha, i+\frac{1}{2}}^{p+1} \equiv \frac{\widehat{\Gamma}_{\|, \alpha, i}^{p+1}+\widehat{\Gamma}_{\|, \alpha, i+1}^{p+1}}{2} .
\end{aligned}
$$

We note that, while these momenta are equivalent in the continuum, choices of discretization and interpolation for the physical acceleration operator mean this will generally not be so in the discrete. The average current density $\bar{j}_{\|}^{p+1}$ must be based on this same particle flux density $\left(\widehat{\Gamma}_{\|, \alpha, i+\frac{1}{2}}^{p+1}\right)$ and is calculated as

$$
\bar{j}_{\|}^{p+1} \equiv \frac{1}{N_{x}} \sum_{i}^{N_{x}}\left(\sum_{\alpha}^{N_{s p}} q_{\alpha} \widehat{\Gamma}_{\|, \alpha, i+\frac{1}{2}}^{p+1}\right) .
$$

\section{Discrete conservation strategy for charge, momentum, and energy}

As we saw in Sec. 2.2, there are certain symmetries of the continuum equations that must be satisfied in order to conserve charge, momentum, and energy. For an arbitrary discretization, these symmetries will pose conflicting constraints. As a result, it will not generally be possible to satisfy all of them simultaneously unless we design our discretization such that it includes elements that ensure these properties. In the following development, we will present the discrete definitions of the constraints $\left(\xi, \alpha, \gamma_{q}, \gamma_{t}, \gamma_{x}\right)$ that will enforce the symmetries discussed in Sec.2.2 in the discrete system. For detailed derivations of these constraints, interested readers are referred to Appendix D. Appendix E. and Ref. [31].

\subsection{Discrete definition of $\gamma_{t}$ and $\gamma_{x}$}

In Sec. 2.2, we saw that there are certain continuum identities [Eqs. [14] and [15] ] that must be satisfied regarding the inertial terms coming from the velocity-space transformation. In the discrete, these are used to define the nonlinear constraint functions $\gamma_{t}$ and $\gamma_{x}$. From Eq. [14], to obtain discrete momentum conservation, $\gamma_{t}$ must satisfy

$$
\begin{aligned}
& \left\langle v_{\|, \alpha, i, j}^{p}, \delta_{t} \tilde{f}_{\alpha, i, j, k}^{p+1}\right\rangle_{\delta \boldsymbol{c}}-\left\langle 1, \delta_{t}\left(v_{\|, \alpha, i, j} \tilde{f}_{\alpha, i, j, k}\right)^{p+1}\right\rangle_{\delta \boldsymbol{c}} \\
& -\left\langle v_{\|, \alpha, i, j}^{p}, \frac{1}{v_{\alpha, i}^{*, p}} \delta_{\boldsymbol{c}} \cdot\left[\gamma_{t, \alpha, i}^{p+1} \delta_{t}\left(\boldsymbol{v}_{\alpha, i}\right)^{p+1} \overline{\left(\tilde{f}_{\alpha}^{p+1}\right)_{i} \delta_{t}(\boldsymbol{v})}\right]_{j, k}\right\rangle_{\delta \boldsymbol{c}}=0,
\end{aligned}
$$


while $\gamma_{x}$ must satisfy

$$
\begin{aligned}
\left\langle v_{\|, \alpha, i, j}^{p}-\right. & \left.v_{\|, \alpha, i+1, j}^{p}, \frac{1}{\Delta x} v_{\alpha, i+\frac{1}{2}}^{*, p} \hat{v}_{\|, \mathrm{eff}, \alpha, i+\frac{1}{2}, j}^{p+1} \overline{\left(\tilde{f}_{\alpha}^{p+1}\right)_{i+\frac{1}{2}, j, k}^{\hat{v}_{\|, \mathrm{eff}}}}\right\rangle_{\delta \boldsymbol{c}} \\
& \left.-\left\langle v_{\|, \alpha, i, j}^{p}, \frac{1}{2 v_{\alpha, i}^{*, p} \delta_{\boldsymbol{c}} \cdot\left[\gamma_{x, \alpha, i+\frac{1}{2}}^{p+1} v_{\alpha, i+\frac{1}{2}}^{*, p} \hat{v}_{\|, \mathrm{eff}, i+\frac{1}{2}} \delta_{x}\left(\boldsymbol{v}^{p}\right)_{i+\frac{1}{2}} \overline{\left(\tilde{f}_{\alpha}^{p+1}\right)_{i}} v_{\|, \mathrm{eff}} \delta_{x}(\boldsymbol{v})\right.}\right]_{j, k}\right\rangle_{\delta \boldsymbol{c}} \\
& -\left\langle v_{\|, \alpha, i+1, j}^{p}, \frac{1}{2 v_{\alpha, i+1}^{*, p}} \delta_{\boldsymbol{c}} \cdot\left[\gamma_{x, \alpha, i+\frac{1}{2}}^{p+1} v_{\alpha, i+\frac{1}{2}}^{*, p} \hat{v}_{\|, \mathrm{eff}, i+\frac{1}{2}} \delta_{x}\left(\boldsymbol{v}^{p}\right)_{i+\frac{1}{2}} \overline{\left(\tilde{f}_{\alpha}^{p+1}\right)_{i, \mathrm{eff}} \delta_{x}(\boldsymbol{v})}\right]_{j, k}\right\rangle_{\delta \boldsymbol{c}}=0 .
\end{aligned}
$$

To obtain discrete energy conservation, according to Eq. 15 $\gamma_{t}$ must satisfy

$$
\begin{aligned}
\left\langle m_{\alpha} \frac{1}{2}\left(\boldsymbol{v}_{\alpha, i, j, k}^{p}\right)^{2}, \delta_{t} \tilde{f}_{\alpha, i, j, k}^{p+1}\right\rangle_{\delta \boldsymbol{c}} & -\left\langle 1, \delta_{t}\left(\frac{1}{2}\left(\boldsymbol{v}_{\alpha, i, j, k}\right)^{2} \tilde{f}_{\alpha, i, j, k}\right)^{p+1}\right\rangle_{\delta \boldsymbol{c}} \\
& -\left\langle m_{\alpha} \frac{1}{2}\left(\boldsymbol{v}_{\alpha, i, j, k}^{p}\right)^{2}, \frac{1}{v_{\alpha, i}^{*, p}} \delta_{\boldsymbol{c}} \cdot\left[\gamma_{t, \alpha, i}^{p+1} \delta_{t}\left(\boldsymbol{v}_{\alpha, i}\right)^{p+1} \overline{\left(\tilde{f}_{\alpha}^{p+1}\right)_{i}^{\delta_{t}(\boldsymbol{v})}}\right]_{j, k}\right\rangle_{\delta \boldsymbol{c}}=0,
\end{aligned}
$$

while $\gamma_{x}$ must satisfy

$$
\begin{aligned}
& \left\langle\frac{\left(\boldsymbol{v}_{\alpha, i, j, k}^{p}\right)^{2}}{2}-\frac{\left(\boldsymbol{v}_{\alpha, i+1, j, k}^{p}\right)^{2}}{2}, \frac{1}{\Delta x} v_{\alpha, i+\frac{1}{2}}^{*, p} \hat{v}_{\|, \mathrm{eff}, \alpha, i+\frac{1}{2}, j}^{p+1} \overline{\left(\tilde{f}_{\alpha}^{p+1}\right)_{i+\frac{1}{2}, j, k}}\right\rangle_{\delta \boldsymbol{c}} \\
& -\left\langle\frac{\left(\boldsymbol{v}_{\alpha, i, j, k}^{p}\right)^{2}}{2}, \frac{1}{2 v_{\alpha, i}^{*, p}} \delta_{\boldsymbol{c}} \cdot\left[\gamma_{x, \alpha, i+\frac{1}{2}}^{p+1} v_{\alpha, i+\frac{1}{2}}^{*, p} \hat{v}_{\|, \mathrm{eff}, i+\frac{1}{2}} \delta_{x}\left(\boldsymbol{v}^{p}\right)_{i+\frac{1}{2}} \overline{\left(\tilde{f}_{\alpha}^{p+1}\right)_{i}} v_{\|, \mathrm{eff}} \delta_{x}(\boldsymbol{v})\right]_{j, k}\right\rangle_{\delta \boldsymbol{c}} \\
& -\left\langle\frac{\left(\boldsymbol{v}_{\alpha, i+1, j, k}^{p}\right)^{2}}{2}, \frac{1}{2 v_{\alpha, i+1}^{*, p}} \delta_{\boldsymbol{c}} \cdot\left[\gamma_{x, \alpha, i+\frac{1}{2}}^{p+1} v_{\alpha, i+\frac{1}{2}}^{*, p} \hat{v}_{\|, \mathrm{eff}, i+\frac{1}{2}} \delta_{x}\left(\boldsymbol{v}^{p}\right)_{i+\frac{1}{2}} \overline{\left(\tilde{f}_{\alpha}^{p+1}\right)_{i+1}} v_{\|, \mathrm{eff}} \delta_{x}(\boldsymbol{v})\right]_{j, k}\right\rangle_{\delta \boldsymbol{c}}=0 .
\end{aligned}
$$

The constraint functions $\gamma_{t}$ and $\gamma_{x}$ are expanded in velocity-space basis functions, e.g.,

$$
\gamma_{t}=1+\sum_{r=0}^{P_{\|}} \sum_{s=0}^{P_{\perp}} C_{r, s} B_{\|, r}\left(c_{\|}\right) B_{\perp, s}\left(c_{\perp}\right),
$$

where the $B_{\|, r}\left(c_{\|}\right), B_{\perp, s}\left(c_{\perp}\right)$ are the $r^{t h}$ and $s^{t h}$ velocity-space functions in $c_{\|}$and $c_{\perp}$, respectively, in some chosen basis (in this work, we use a Fourier representation as in [31]). The $C_{r, s}$ are the corresponding coefficient weights, which are obtained via the solution of a constrained-minimization problem using Eqs. (14)- $(15)$. More details on the approach can be found in Ref. [31]. We note here that the solution is relatively inexpensive, involving the computation of the discrete moments in Eqs. $147-\sqrt{15}$, and the solution of a straightforward linear system of the size $\mathcal{O}\left(P_{\|} P_{\perp}\right) \sim$ $\mathcal{O}(10)-\mathcal{O}(100)$. Note that $\gamma_{x}$ depends on $\xi$ through $\hat{v}_{\|, \alpha, \text { eff }}$, and so must be calculated after $\xi$ is obtained. Together, these constraint functions ensure that the integration by parts and product rule that produce Eqs. (14)-(15) are upheld discretely.

\subsection{Discrete definition of $\xi$, $\phi$, and $\gamma_{q}$}

The constraint function $\xi$ is defined by

$$
\xi_{\alpha, i+\frac{1}{2}}^{p+1}=\frac{\widehat{\Gamma}_{\|, \alpha, i+\frac{1}{2}}^{p+1}-\widetilde{\Gamma}_{\|, \alpha, i+\frac{1}{2}}^{p+1}}{\Pi_{\xi, \|, \alpha, i+\frac{1}{2}}^{p+1}} .
$$


The quantity $\widehat{\Gamma}_{\|, \alpha, i+\frac{1}{2}}^{p+1}$ is defined in Eqs. $35 p-36$, and $\widetilde{\Gamma}_{\|, \alpha, i+\frac{1}{2}}^{p+1}$ and $\Pi_{\xi, \|, \alpha, i+\frac{1}{2}}^{p+1}$ are given by:

$$
\begin{gathered}
\widetilde{\Gamma}_{\|, \alpha, i+\frac{1}{2}}^{p+1} \equiv \frac{1}{m_{\alpha}}\left\langle m_{\alpha}, v_{\alpha, i+\frac{1}{2}}^{*, p}\left(c_{\|, j}+\hat{u}_{\|, \alpha, i+\frac{1}{2}}^{*, p}\right) \overline{\left.\left(\tilde{f}_{\alpha}^{p+1}\right)^{v_{\|+, i+\frac{1}{2}, j, j}^{p}}\right\rangle_{\delta c},}\right. \\
\Pi_{\xi, \|, \alpha, i+\frac{1}{2}}^{p+1} \equiv \frac{1}{m_{\alpha}}\left\langle m_{\alpha}, v_{\alpha, i+\frac{1}{2}}^{*, p}\left|c_{\|, j}+\hat{u}_{\|, \alpha, i+\frac{1}{2}}^{*, p}\right| \overline{\left.\left(\tilde{f}_{\alpha}^{p+1}\right)_{i+\frac{1}{2}, j, k}^{\xi_{\alpha, i+\frac{1}{2}}^{p+1}}\right\rangle_{\delta c},}\right.
\end{gathered}
$$

where the integrals in the scalar product are obtained from the fluxes in terms $(a)$ and $(c)$ in Eq. 23. The action of the constraint function $\xi$ is to ensure that Gauss's law is upheld [see Eq. [16], and is generally described as the "charge-conserving" constraint.

The constraint functions $\phi$ and $\gamma_{q}$ are split in velocity space using the following convention:

$$
\begin{aligned}
\phi_{\alpha, i, j+\frac{1}{2}}^{p+1} & = \begin{cases}\phi_{\alpha, i}^{+, p+1} & \text { if } v_{\|, \alpha, i, j+\frac{1}{2}} \geq u_{\|, \alpha, i}^{p}, \\
1 & \text { otherwise }\end{cases} \\
\gamma_{q, \alpha, i, j+\frac{1}{2}}^{p+1} & = \begin{cases}1 & \text { if } v_{\|, \alpha, i, j+\frac{1}{2}} \geq u_{\|, \alpha, i}^{p}, \\
\gamma_{q, \alpha, i}^{-, p+1} & \text { otherwise }\end{cases}
\end{aligned}
$$

where

$$
u_{\|, \alpha, i}^{p}=\frac{\left\langle v_{\|}, \tilde{f}_{\alpha, i, j, k}^{p+1}\right\rangle_{\delta c}}{\left\langle 1, \tilde{f}_{\alpha, i, j, k}^{p+1}\right\rangle_{\delta c}}
$$

is the bulk velocity of species $\alpha$ from the previous timestep. The quantities $\phi_{\alpha, i}^{+, p+1}$ and $\gamma_{q, \alpha, i}^{-, p+1}$ are thus coupled by the $2 \times 2$ linear system

$$
\left[\begin{array}{cc}
n_{\alpha, i}^{+, p+1} & n_{\alpha, i}^{-, p+1} \\
\Gamma_{\|, \alpha, i}^{+, p+1} & \Gamma_{\|,,, p+i}^{-, p+1}
\end{array}\right]\left[\begin{array}{c}
\phi_{\alpha, i}^{+, p+1}+1 \\
\gamma_{q, p, i}^{-, p+1}+1
\end{array}\right]=\left[\begin{array}{c}
\left(n_{\alpha, i}^{p+1}-\widehat{n}_{\alpha, i}^{p+1}\right) E_{\|, i}^{p+1} \frac{q_{\alpha}}{m_{\alpha}} \\
\frac{\epsilon_{0}}{m_{\alpha} N_{s p}}\left[E_{\|, i+\frac{1}{2}}^{p+1} \delta_{t} E_{\|, i+\frac{1}{2}}^{p+1}-\delta_{t}\left(\frac{1}{2} E_{\|, i+\frac{1}{2}}^{2}\right)^{p+1}\right.
\end{array}\right],
$$

which is well-posed (see Appendix F) and may be easily inverted analytically to calculate $\phi$ and $\gamma_{q}$. In the preceding, we defined the discrete number densities

$$
\begin{aligned}
& n_{\alpha, i}^{p+1} \equiv\left\langle 1, \tilde{f}_{\alpha, i, j, k}^{p+1}\right\rangle_{\delta \boldsymbol{c}},
\end{aligned}
$$

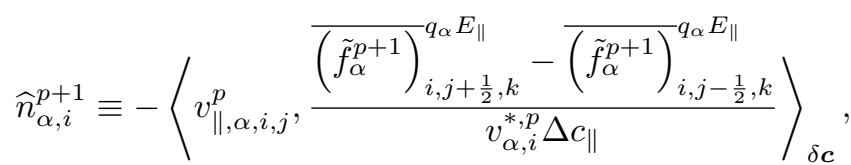

which come from the direct $v_{\|}^{0}$ moment of $\tilde{f}_{\alpha}$, and the $v_{\|}$moment of the electrostatic acceleration operator, respectively. We also defined the "upper" and "lower" densities

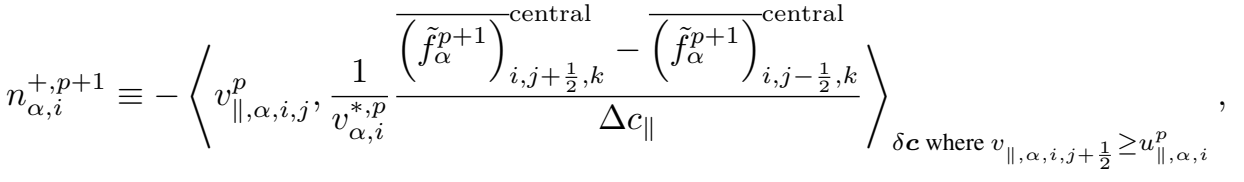

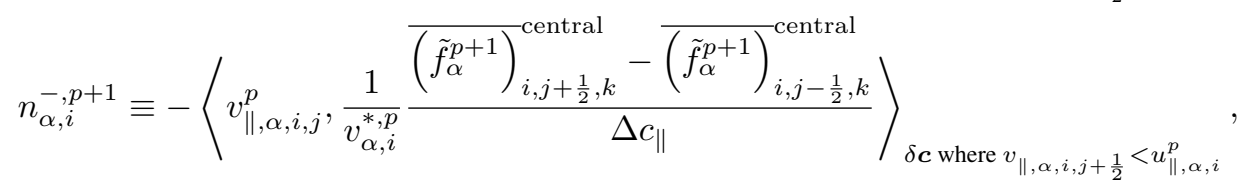


which come from the appropriate $m_{\alpha} v_{\|}$"half moments" of the pseudo-operators associated with $\phi$ and $\gamma_{q}$, as well as the upper and lower momenta

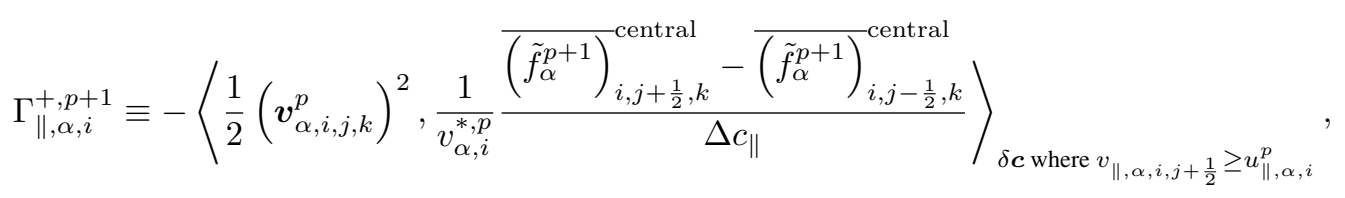

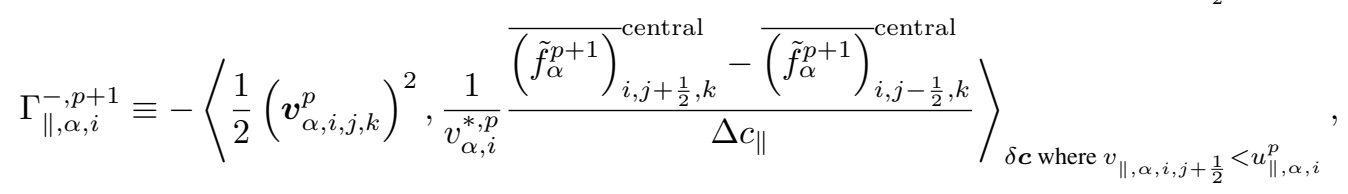

from the $\frac{1}{2} m_{\alpha} \boldsymbol{v}^{2}$ half moments of the same operators. The constraint functions $\phi$ and $\gamma_{q}$ together act to enforce the symmetries in Eqs. (17) and (18), which lead to momentum and energy conservation.

The nonlinear constraint function approach has been employed previously for actively enforcing conservation for the Vlasov-Ampère system [20, 30] with a symplectic time-integration scheme as well as for the Vlasov-Fokker-Planck system with a velocity-space adaptive transformation [11, 31]. Here, this approach has been applied to the velocityspace transformed Vlasov-Ampère system with BDF2 temporal discretization, though it may in principle be applied to a wide variety of temporal discretizations. Here, we further point out that all the constraint functions $\left(\gamma_{t}, \gamma_{x}, \xi, \phi, \gamma_{q}\right)$ are calculated locally in configuration space, and are almost entirely independent of one another. The single exception is the dependence of $\gamma_{x}$ on $\xi$ through the quantity $v_{\|, \text {eff }}$, which is satisfied simply by calculating $\xi$ before $\gamma_{x}$.

\section{Solving the discretized Vlasov-Ampère system}

To solve the discretized Vlasov-Ampère system, we use the high-order/low-order (HOLO) nonlinear acceleration iterative strategy [26]. HOLO accelerates the nonlinear convergence of the temporally implicit high-order (HO) Vlasov-Ampère system through a low-order (LO) representation, which efficiently exposes the stiff physics. The LO system is obtained from the velocity-space moments of the HO system. This approach has been successfully employed to solve the Vlasov-Ampère and Vlasov-Fokker-Planck-Ampère systems among many other problems [13, 20, 2630].

\subsection{Formulation, discretization, and solution of the LO system}

In our context, the LO equations (moments of the species' Vlasov equations) are used to provide a well-informed guess for the electric field $E_{\|}$, which results in fast nonlinear convergence of the original Vlasov-Ampère system. The LO moment-Ampère system allows for the stiff time-scales associated with collective physics (e.g., plasma waves) to be efficiently captured in a lower-dimensional system. A key component of the strategy is the enslavement of the discretization error and any missing physics in the LO system through discrete consistency terms. This ensures that the $\mathrm{LO}$ and $\mathrm{HO}$ moments agree exactly upon nonlinear convergence.

To obtain the LO quantities and their respective equations, we take the appropriate velocity-space moments of the Vlasov equation in the continuum:

$$
\langle\cdot, \cdot\rangle_{\boldsymbol{c}} \equiv 2 \pi \int_{-\infty}^{\infty} d c_{\|} \int_{0}^{\infty} c_{\perp} d c_{\perp}(\cdot * \cdot) .
$$

The evolution of $n_{\alpha}$ and $\Gamma_{\|, \alpha}$ are described by the corresponding moments of the Vlasov equation, Eq.(10):

$$
\begin{aligned}
& \langle 1, \text { Vlasov }\rangle_{\boldsymbol{c}}=\quad \partial_{t} n_{\alpha}+\partial_{x}\left(\Gamma_{\|, \alpha}\right)=\quad 0, \\
& \left\langle v_{\|}, \text {Vlasov }\right\rangle_{\boldsymbol{c}}=\partial_{t}\left(\Gamma_{\|, \alpha}\right)+\partial_{x} S_{\|\|, \alpha}^{(2)}-\frac{q_{\alpha}}{m_{\alpha}} n_{\alpha} E_{\|}=0 .
\end{aligned}
$$

In Eq. 56 , the quantity $S_{\|\|, \alpha}^{(2)}$ is the $v_{\|}^{2}$ moment of $\tilde{f}_{\alpha}$.

Thus, the LO system consists of the moment equations together with Ampère's equation: 


$$
\begin{aligned}
\epsilon_{0} \partial_{t} E_{\|}^{L O}+\sum_{\alpha} q_{\alpha} \Gamma_{\|, \alpha}^{L O} & =\bar{j}_{\|}, \\
\partial_{t} n_{\alpha}^{L O}+\partial_{x}\left(\Gamma_{\|, \alpha}^{L O}\right) & =\eta_{n_{\alpha}}^{H O}, \\
\partial_{t}\left(\Gamma_{\|, \alpha}^{L O}\right)+\partial_{x}\left(n_{\alpha}^{L O} \widetilde{S^{(2)}}{ }_{\|\|, \alpha}^{H O}\right)-\frac{q_{\alpha}}{m_{\alpha}} n_{\alpha}^{L O} E_{\|}^{L O} & =\eta_{n u_{\|, \alpha}}^{H O} .
\end{aligned}
$$

In Eqs. 58 and 59 we have introduced the HO consistency terms, $\eta_{n_{\alpha}}^{H O}$ and $\eta_{n u_{\|, \alpha}}^{H O}$, which enslave the truncation error (and any missing physics) of the LO system to the HO system (to be explicitly defined later). Note that, to provide a closure for the higher-order moments to the LO system, we use in Eq. 59] the density-normalized total stress tensor from the $\mathrm{HO}$ system,

$$
{\widetilde{S^{(2)}}}_{\|\|, \alpha}^{H O}=\frac{\left\langle v_{\|}^{2}, f_{\alpha}\right\rangle_{\boldsymbol{c}}}{\left\langle 1, f_{\alpha}\right\rangle_{\boldsymbol{c}}} .
$$

The presence of the LO density $n_{\alpha}^{L O}$ in the LO momentum advection exposes the stiff isothermal wave in the LO system [13, 20].

The LO system is discretized on a staggered finite-difference grid, where we define the density, $n_{\alpha, i}^{L O}$, at cell centers and the particle number density flux, $n u_{\|, \alpha, i+\frac{1}{2}}^{L O}$, and electric field, $E_{\|, i+\frac{1}{2}}^{L O}$, at cell faces. The discrete form of the LO system is

$$
\begin{aligned}
& R_{n_{\alpha}, i}^{l} \equiv \frac{b^{p+1} n_{\alpha, i}^{L O, p+1, l}+b^{p} n_{\alpha, i}^{H O, p}+b^{p-1} n_{\alpha, i}^{H O, p-1}}{\Delta t^{p}}+\frac{\Gamma_{\|, \alpha, i+\frac{1}{2}}^{L O, p+1, l}-\Gamma_{\|, \alpha, i-\frac{1}{2}}^{L O, p+1, l}}{\Delta x}-\eta_{n_{\alpha}, i}^{l-1} \\
& R_{n u_{\|, \alpha}, i+\frac{1}{2}}^{l} \equiv \frac{b^{p+1} \Gamma_{\|, \alpha, i+\frac{1}{2}}^{L O, p+1, l}+b^{p} \Gamma_{\|, \alpha, i+\frac{1}{2}}^{H O, p}+b^{p-1} \Gamma_{\|, \alpha, i+\frac{1}{2}}^{H O, p-1}}{\Delta t^{p}}
\end{aligned}
$$

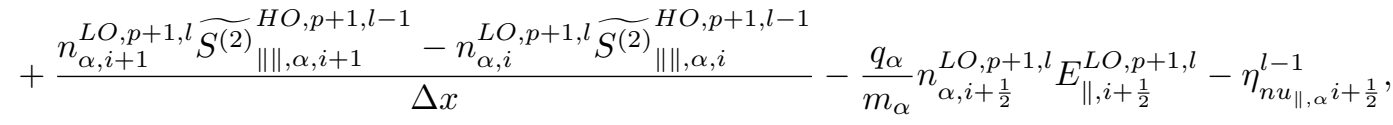

$$
\begin{aligned}
& R_{E_{\|}, i+\frac{1}{2}}^{l} \equiv \epsilon_{0} \frac{b^{p+1} E_{\|, i+\frac{1}{2}}^{L O, p+1, l}+b^{p} E_{\|, i+\frac{1}{2}}^{L O, p}+b^{p-1} E_{\|, i+\frac{1}{2}}^{L O, p-1}}{\Delta t^{p}}+\sum_{\alpha} q_{\alpha} \Gamma_{\|, \alpha, i+\frac{1}{2}}^{L O, p+1, l}-\bar{j}_{\|}^{p+1, l} .
\end{aligned}
$$

In Eqs. (61)- 63), the quantities $R_{n_{\alpha}, i}, R_{n u_{\|, \alpha}, i+\frac{1}{2}}$, and $R_{E_{\|}, i+\frac{1}{2}}$ are the nonlinear residuals for the corresponding LO quantities (which should be converged to zero), $i$ is the configuration space index, and $l$ is the HOLO iteration index. The quantity $n_{\alpha, i+\frac{1}{2}}^{L O, p+1, l}$ is the cell-centered density linearly interpolated to cell faces. $\widetilde{S^{(2)}}{ }_{\|\|, \alpha, i}$ is defined at cell centers. The HO quantities are generally defined as the corresponding direct moments of the distribution $\tilde{f}_{\alpha, i, j, k}^{p+1, l}$

$$
\begin{aligned}
n_{\alpha, i}^{H O, p+1, l} & =\left\langle 1, \tilde{f}_{\alpha, i}^{p+1, l}\right\rangle_{\delta \boldsymbol{c}} \\
{\widetilde{S^{(2)}}}_{\|\|, \alpha}^{H O, p+1, l} & =\frac{\left\langle v_{\|, j}^{2}, \tilde{f}_{\alpha, i}^{p+1, l}\right\rangle_{\delta \boldsymbol{c}}}{\left\langle 1, \tilde{f}_{\alpha, i}^{p+1, l}\right\rangle_{\delta \boldsymbol{c}}}
\end{aligned}
$$

The exception is the cell-face HO particle flux density used in the LO system,

$$
\Gamma_{\|, \alpha, i+\frac{1}{2}}^{H O, p+1, l}=\widehat{\Gamma}_{\|, \alpha, i+\frac{1}{2}}^{p+1, l}
$$

which is taken to be the same as the flux that forms the current for Ampère's equation $\left[\widehat{\Gamma}_{\|, \alpha, i+\frac{1}{2}}^{p+1, l}\right.$ is defined as in Eqs. [35]-36]. We note here that, for the HOLO system, we use a slightly different definition for the discrete averaged 
current, $\bar{j}_{\|}^{p+1, l}$, than in Eq. 37 , based on the cell-face parallel LO particle flux density:

$$
\bar{j}_{\|}^{p+1, l} \equiv \frac{1}{N_{x}} \sum_{i}^{N_{x}}\left(\sum_{\alpha}^{N_{s p}} q_{\alpha} \Gamma_{\|, \alpha, i+\frac{1}{2}}^{L O, p+1, l}\right) .
$$

The discrete consistency terms, $\eta_{n_{\alpha}, i}^{l}$ and $\eta_{n u_{\|, \alpha}, i+\frac{1}{2}}^{l}$, are defined by introducing HO moments into the LO equations as

$$
\begin{aligned}
& \eta_{n_{\alpha}, i}^{l} \equiv \frac{b^{p+1} n_{\alpha, i}^{H O, p+1, l}+b^{p} n_{\alpha, i}^{H O, p}+b^{p-1} n_{\alpha, i}^{H O, p-1}}{\Delta t^{p}}+\frac{\Gamma_{\|, \alpha, i+\frac{1}{2}}^{H O, p+1, l}-\Gamma_{\|, \alpha, i-\frac{1}{2}}^{H O, p+1, l}}{\Delta x}-\left\langle 1, R_{\tilde{f}, \alpha, i}^{l}\right\rangle_{\delta \boldsymbol{c}} \\
& \eta_{n u_{\|, \alpha}, i+\frac{1}{2}}^{l} \equiv \frac{b^{p+1} \Gamma_{\|, \alpha, i+\frac{1}{2}}^{H O, p+1, l}+b^{p} \Gamma_{\|, \alpha, i+\frac{1}{2}}^{H O, p}+b^{p-1} \Gamma_{\|, \alpha, i+\frac{1}{2}}^{H O, p-1}}{\Delta t^{p}}+\frac{n_{\alpha, i+1}^{H O, p+1, l} \widetilde{S^{(2)}}{ }_{\|\|, \alpha, i+1}^{H O, p+1, l}-n_{\alpha, i}^{H O} \widetilde{S^{(2)}}{ }_{\|\|, \alpha, i}^{H O, p+1, l}}{\Delta x} \\
& -\frac{q_{\alpha}}{m_{\alpha}} n_{\alpha, i+\frac{1}{2}}^{H O, p+1, l} E_{\|, i+\frac{1}{2}}^{L O, p+1, l-1}-\frac{\left(\left\langle v_{\|, j}, R_{\tilde{f}, \alpha, i}^{l}\right\rangle_{\delta c}+\left\langle v_{\|, j}, R_{\tilde{f}, \alpha, i+1}^{l}\right\rangle_{\delta c}\right)}{2} .
\end{aligned}
$$

The consistency terms will converge towards zero along with the residuals as the HOLO iteration progresses. In Eqs. $67-68,\left\langle 1, R_{\tilde{f}, \alpha, i}^{l}\right\rangle_{\delta c}$ and $\left\langle v_{\|, j}, R_{\tilde{f}, \alpha, i}^{l}\right\rangle_{\delta c}$ indicate moments of the HO system residual, Eq. 72 , which will be discussed in detail in Sec.5.2

The coupled LO system is solved with an Anderson-accelerated nonlinear quasi-Newton iteration [43, 44]. The quasi-Newton iteration is preconditioned with a direct solution of the linearized moment equations:

$$
\begin{gathered}
\frac{b^{p+1} \delta n_{\alpha, i}}{\Delta t^{p}}+\frac{\delta \Gamma_{\|, \alpha, i+\frac{1}{2}}-\delta \Gamma_{\|, \alpha, i-\frac{1}{2}}}{\Delta x}=-R_{n_{\alpha}, i}^{l}, \\
\frac{b^{p+1} \delta \Gamma_{\|, \alpha, i+\frac{1}{2}}}{\Delta t^{p}}+\frac{\delta n_{\alpha, i+1} \widetilde{S^{(2)}}{ }_{\|\|, \alpha, i+1}^{H O, p+1, l-1}-\delta n_{\alpha, i} \widetilde{S}^{(2)}{ }_{\|\|, \alpha, i}^{H O, p+1, l-1}}{\Delta x} \\
-\frac{q_{\alpha}}{m_{\alpha}}\left(\delta n_{\alpha, i+\frac{1}{2}} E_{\|, i+\frac{1}{2}}^{L O, p+1, l}+n_{\alpha, i+\frac{1}{2}}^{L O, p+1, l} \delta E_{\|, i+\frac{1}{2}}\right)=-R_{n u_{\|, \alpha}, i+\frac{1}{2}}^{l}, \\
\epsilon_{0} \frac{b^{p+1} \delta E_{\|, i+\frac{1}{2}}}{\Delta t^{p}}+\sum_{\alpha} q_{\alpha} \delta \Gamma_{\|, \alpha, i+\frac{1}{2}}=-R_{E_{\|}, i+\frac{1}{2}}^{l} .
\end{gathered}
$$

After substitution of Eqs. (69) and (71) into Eq. (70) for $\delta n_{\alpha}$ and $\delta E$, respectively, the system reduces to a single equation for $\delta \Gamma_{\|, \alpha, i+\frac{1}{2}}$ at cell faces, coupled in space and across species. After linear inversion for $\delta \Gamma_{\|, \alpha, i+\frac{1}{2}}, \delta n_{\alpha, i}$ and $\delta E_{\|, i+\frac{1}{2}}$ are found directly from Eqs. $\sqrt[69 p]{ }$ and 771 . To accelerate HOLO convergence further, an additional layer of Anderson acceleration is wrapped around the outer HOLO iteration driven by to the LO solution, similarly to what was considered in Ref. [45].

\subsection{Discretization and solution of the HO system}

The HO system is discretized essentially as presented in Sec. 3. with the result reproduced here to highlight the coupling with the LO system: 


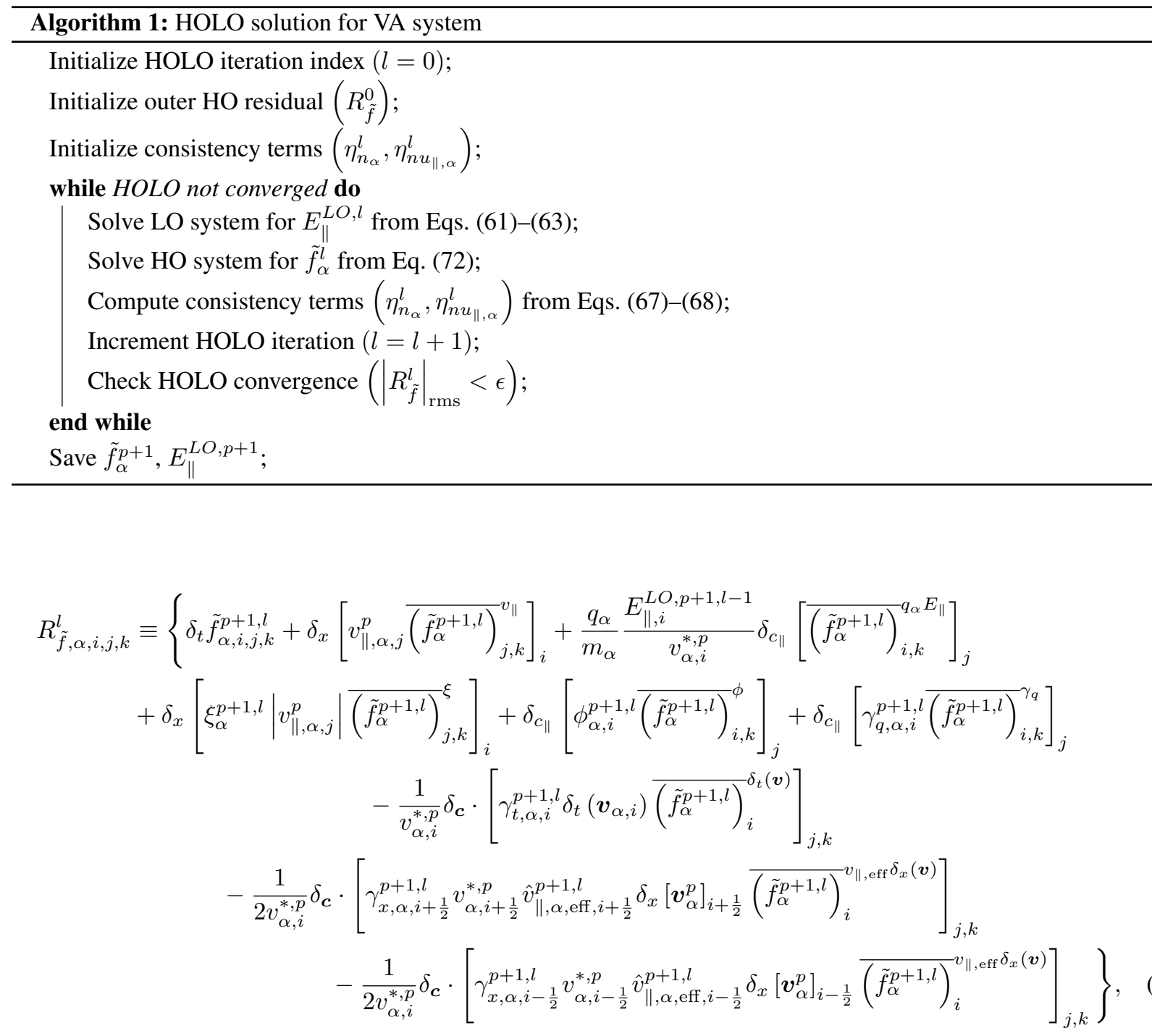

where

$$
\hat{v}_{\|, \alpha, \text { eff }, i+\frac{1}{2}, j}^{p+1, l}=\left(c_{\|, j}+\hat{u}_{\|, \alpha, i+\frac{1}{2}}^{*, p}\right)+\xi_{\alpha, i+\frac{1}{2}}^{p+1, l}\left|c_{\|, j}+\hat{u}_{\|, \alpha, i+\frac{1}{2}}^{*, p}\right| .
$$

The quantity $R_{\tilde{f}, \alpha, i, j, k}^{l}$ is the $\mathrm{HO}$ system residual. Note that we have included the superscript $l$ for the HOLO iteration index. Observe that the electric field, $E_{\|}^{L O}$, in Eq. 72 is obtained from the solution of the LO system, which effectively Picard-linearizes the individual Vlasov equations (HO system) in $\tilde{f}_{\alpha}$ and is key for effective nonlinear convergence acceleration. However, the discretization scheme employed in Eq. (72) may still include significant nonlinearities in the advective terms. Thus, the HO system is also solved with Anderson acceleration. For preconditioning, Eq. (72) is linearized in $\delta f_{\alpha, i}$, with a linear upwind discretization for all operators. The system is then solved with the multigridpreconditioned Flexible Generalized Minimal RESiduals (FGMRES) method [46].

\subsection{HOLO solution algorithm}

Thus, the coupled HOLO system is represented by 1) the HO system, which consists a system of the species' Vlasov equations, Eq. (72), and 2) the LO system, which consists of the moment equations for each species' mass and momentum and Ampère's equation, Eqs. 61]-663). Algorithm 11depicts the HOLO-accelerated iteration. Convergence is measured through the root-mean-square (rms) of the $\mathrm{HO}$ residual vector,

$$
\left|R_{\tilde{f}}^{l}\right|_{\mathrm{rms}}=\sqrt{\frac{1}{N_{\alpha} N_{x} N_{y} N_{z}} \sum_{\alpha=1}^{N_{s p}} \sum_{i=1}^{N_{x}} \sum_{j=1}^{N_{y}} \sum_{k=1}^{N_{z}}\left(R_{\tilde{f}, \alpha, i, j, k}^{l}\right)^{2} .}
$$


Here, the convergence tolerance, $\epsilon$, is defined as

$$
\epsilon=\epsilon_{a}+\epsilon_{r}\left|R_{\tilde{f}}^{0}\right|_{\mathrm{rms}},
$$

where $\epsilon_{a}$ is an absolute tolerance and $\epsilon_{r}$ is the relative tolerance.

\section{Numerical results}

In this section, we demonstrate the accuracy, convergence, and conservation properties of the proposed numerical scheme. We do so using several canonical collisionless problems of increasing complexity, ranging from the linear Landau damping to an ion-acoustic shock wave. Unless specified otherwise, for all the problems presented we normalize the particle mass and charge to the electron mass, $m_{e}$, and proton charge, $q_{p}$, while normalizing the temperature, density, velocity, and time to the reference temperature, $T_{0}$, density, $n_{0}$, speed, $v_{0}=\sqrt{\left(T_{0} / m_{e}\right)}$, and time-scale, $\tau_{0}=\omega_{p, e}^{-1}$ (where $\omega_{p, e}$ is the electron plasma frequency). The initial velocity distributions for each species, $\alpha$, are assumed to be normalized Maxwellians

$$
\tilde{f}_{M, \alpha}=\frac{n_{\alpha}}{\pi^{3 / 2}}\left(\frac{v_{\alpha}^{*}}{v_{t h, \alpha}}\right)^{3} \exp \left[-\frac{1}{v_{t h, \alpha}^{2}}\left(v_{\alpha}^{*}\left(\boldsymbol{c}+\hat{u}_{\|, \alpha}^{*} \boldsymbol{e}_{\|}\right)-u_{\|, \alpha} \boldsymbol{e}_{\|}\right)^{2}\right],
$$

where $v_{t h, \alpha} \equiv \sqrt{\frac{2 T_{\alpha}}{m_{\alpha}}}$. Unless stated otherwise, the velocity-space adaptivity metrics (i.e., the offset velocity $u_{\|, \alpha}^{*}$ and reference speed $v_{\alpha}^{*}$ ) are initialized and spatio-temporally adapted using each species' initial bulk velocity $u_{\|, \alpha}$ and thermal speed $v_{\alpha}^{*}$, respectively. For robustness, some smoothing and limiting strategies are applied to avoid large spatial or temporal gradients in the metrics. See Ref. [31] for specific details. The initial electric field, $E_{\|}$, is determined from the solution of Poisson's equation driven by the initial charge density:

$$
\begin{gathered}
-\epsilon_{0} \frac{\partial^{2} \Phi_{0}}{\partial x^{2}}=\sum_{\alpha}^{N_{s}} q_{\alpha} n_{\alpha}, \\
E_{\|, 0}=-\frac{\partial \Phi_{0}}{\partial x} .
\end{gathered}
$$

A realistic proton-electron mass ratio $m_{i} / m_{e}=1836$ is used for all cases. Unless otherwise specified, the relative nonlinear convergence tolerance is $\epsilon_{r}=10^{-4}$, while the absolute tolerance is set to a low value $\left(\epsilon_{a}=10^{-14}\right)$ to avoid interference with the relative convergence (see Sec. 5.3.

\subsection{Landau damping}

The linear and nonlinear electron Landau damping tests show the ability of the solver to capture fine collisionless features in phase space. For this problem, the rate of oscillation and decay of the electric field energy is determined by the dispersion relation,

$$
1+\frac{1}{k^{2}}\left[1+\frac{\omega}{\sqrt{2} k} Z\left(\frac{\omega}{\sqrt{2} k}\right)\right]=0
$$

which determines the complex frequency $\omega=\omega_{r}-i \gamma$ for a given wavenumber $k$. The function $Z$ is the plasma dispersion function for a Maxwellian [47].

\subsubsection{Linear Landau damping}

To initiate the linear Landau damping in an electron-proton plasma, we impose a sinusoidal density perturbation on electrons with wavenumber $k \lambda_{D}=0.5$ and amplitude $\delta n=0.01$. We expect it to decay at a linear rate of $\gamma=-0.155$ [20, 48]. Both species have the same initial temperature $T_{0}=1$, bulk velocity $u_{\|, 0}=0$, and unperturbed density $n_{0}=1$. The simulation is performed on the mesh $N_{x}=32, N_{v_{\|}}=512, N_{v_{\perp}}=32$, with an average time-step of $\Delta t=0.1 \omega_{p e}^{-1}$. The velocity-space domain is $c_{\|} \in[-6,+6], c_{\perp} \in[0,5]$. The offset velocity for both species is $u_{\|}^{*}=0$, while the species normalization reference speeds are $v_{e, 0}^{*}=\sqrt{\frac{2 T_{0}}{m_{e}}}, v_{i, 0}^{*}=\sqrt{\frac{2 T_{0}}{m_{i}}}$. The configuration space domain size is $L_{x}=4 \pi$. From Fig. 1, we see that the rate of decay matches the linear theory well. 


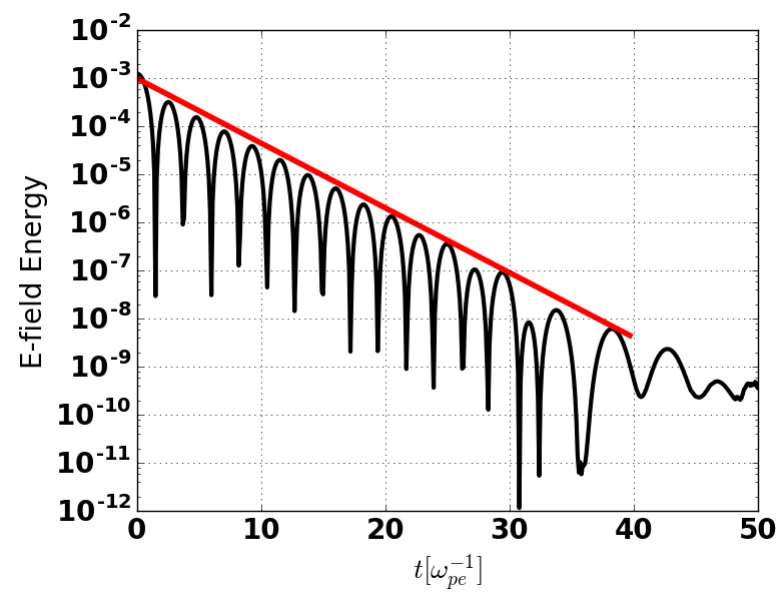

Figure 1: Decay $(\gamma=-0.155)$ of the electric field energy for the linear Landau damping test.

\subsubsection{Numerical convergence of the method}

To demonstrate that the full set of discrete governing equations achieve our desired level of accuracy, we perform a convergence study in time and space. Convergence is measured by computing the $L_{2}$ norm, $L_{2}^{E, \Delta}$, of the difference in the electric field for each solution relative to a reference solution $E_{\|}^{\Delta \text {,ref }}$ obtained with a small time-step or with high resolution in the configuration or velocity space,

$$
L_{2}^{E, \Delta} \equiv \sqrt{\sum_{i}^{N_{x}} \Delta x_{i}\left[\left(E_{\|, i}-E_{\|, i}^{\Delta, \mathrm{ref}}\right)\right]^{2}} .
$$

For the convergence studies, a relative nonlinear convergence tolerance of $\epsilon_{r}=10^{-12}$ is used to resolve the difference in truncation error at small time-steps and fine-grid resolutions. Figure 2 shows convergence with temporal resolution
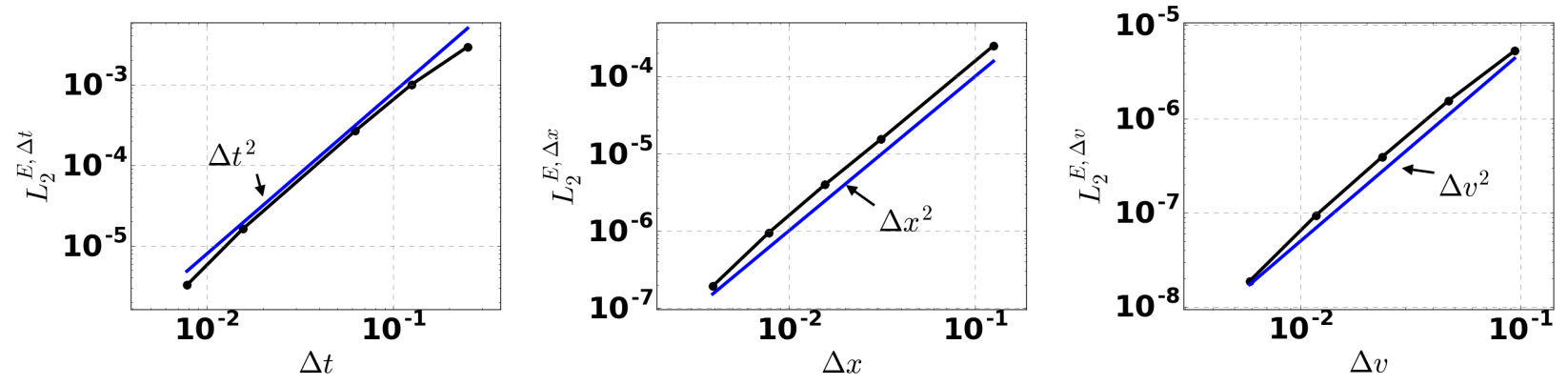

Figure 2: Numerical convergence studies for temporal (left), configuration space (center), and velocity space (right) resolutions.

(left, with a reference time-step of $\Delta t=3.90625 \times 10^{-3}$, and a mesh of $N_{x}=32, N_{v_{\|}}=512, N_{v_{\perp}}=32$ ), configuration space resolution (center, with a reference mesh of $N_{x}=2048$, using a time-step $\Delta t=0.1$, with a velocity-space mesh of $N_{v_{\|}}=512, N_{v_{\perp}}=32$ ), and velocity space resolution (right, with a reference mesh of $N_{v_{\|}}=4096, N_{v_{\perp}}=2048$, using a time-step of $\Delta t=0.1$, and a configuration-space mesh of $N_{x}=32$ ). The maximum simulation time is $t_{\max }=1$ for configuration and velocity-space convergence, and $t_{\max }=10$ for the temporal convergence. As can be seen, second-order convergence rates are observed with respect to all the independent variables.

\subsubsection{Nonlinear Landau damping}

To simulate nonlinear (strong) Landau damping, we again choose $k \lambda_{D}=0.5$, but increase the electron density perturbation magnitude to $\delta n=0.5$. The simulation is performed on a mesh of $N_{x}=256, N_{v_{\|}}=512, N_{v_{\perp}}=32$, 
and the initialization is otherwise identical to the linear Landau damping case. According to the literature, this should produce an initial decay and a subsequent recurrence with rates of $\gamma_{1}=-0.292$ and $\gamma_{2}=0.0815$, respectively. As we

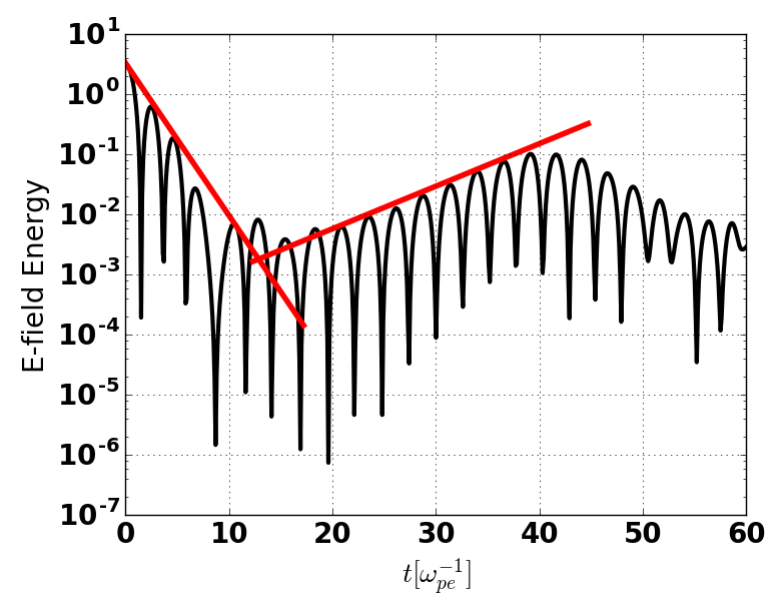

Figure 3: Initial decay $\left(\gamma_{1}=-0.292\right)$ and a subsequent recurrence growth $\left(\gamma_{2}=0.0815\right)$ of the electric field energy for the nonlinear Landau damping test.

see in Fig. (3), the decay and growth of the electric field energy show excellent agreement with other published results for this problem [17, 20].

\subsection{Two-stream instability}

The electron-electron two-stream instability simulation [49] is initialized as two relatively cold, counterstreaming Maxwellian electron beams, each with the bulk velocity $\pm v_{b}$, and with the thermal speed $v_{t h, b}<<v_{b}$, against a neutralizing background of stationary ions. The dispersion relation for this problem is

$$
1+\frac{\omega_{p, b}^{2}}{k^{2} v_{t h, b}^{2}}\left[2+\zeta_{+} Z\left(\zeta_{+}\right)+\zeta_{-} Z\left(\zeta_{-}\right)\right]=0
$$

where

$$
\zeta_{ \pm} \equiv \frac{\omega \mp k v_{b}}{k v_{t h, b}}
$$

and $\omega_{p, b}$ is the beam plasma frequency. In the limit of $v_{t h, b} \rightarrow 0$, Eq. (76) becomes

$$
1-\frac{1}{\left(\omega+v_{b} k\right)^{2}}-\frac{1}{\left(\omega-v_{b} k\right)^{2}}=0
$$

For our simulation, we use electron beam densities of $n_{0}=0.5$, beam velocities $v_{b}= \pm 0.1$ and beam thermal velocities $v_{t h, b} / v_{b}=[0.15,0.3,0.5,0.65,0.8]$. The electron-beam densities are perturbed sinusoidally with wavenumber $k=2 \pi / L_{x}$ and magnitude $\delta n=0.00005$. The domain size is $L_{x}=1$. The velocity-space domain is $c_{\|} \in[-4,+4]$ for $v_{t h, b} / v_{b}=0.15, c_{\|} \in[-5,+5]$ for $v_{t h, b} / v_{b}=0.3$, and $c_{\|} \in[-6,+6]$ for $v_{t h, b} / v_{b} \geq 0.5$, with $c_{\perp} \in[0,5]$. The electrons have an initial offset velocity of $u_{\|, e}^{*}=0$ and normalizing speed of $v_{\alpha, e}^{*}=[0.083,0.087,0.096,0.10,0.114]$. The mesh is $N_{x}=128, N_{v_{\|}}=512, N_{v_{\perp}}=32$. In Fig. 4, we perform a sweep in beam thermal velocity ratio with $\Delta t=0.25 \omega_{p e}^{-1}$, while in Fig. 5 we choose $v_{t h, b} / v_{b}=0.5$ and use $\Delta t=[0.25,1.0,2.0] \omega_{p e}^{-1}$.

Based on the delta-function dispersion relation, Eq. (77), the growth rate of electric field energy is $\gamma=0.353 \omega_{p, b}$. However, for thermalized beams there will be some deviation, and we expect that as the ratio $v_{t h, b} / v_{b}$ increases the system to become less unstable (i.e., $\gamma$ will decrease). Indeed, in Fig. 4 we see that if we increase $v_{t h, b} / v_{b}$ towards some critical ratio near unity, the growth rate decreases precipitously. As shown in Table 1, growth rates calculated from simulations $\left(\gamma_{\text {sim }}\right)$ agree very well with the growth rates obtained from a numerical solution of the dispersion relation for thermalized beams $\left(\gamma_{\text {num }}\right)$ - details of this analysis may be found in Appendix G. In Fig. 5, we show that there is little change in the simulated electric-field growth rate as we vary the time-step size. 


\begin{tabular}{|c|c|c|c|c|c|}
\hline$v_{\text {th }, b} / v_{b}$ & 0.15 & 0.3 & 0.5 & 0.65 & 0.8 \\
\hline \hline$\gamma_{\text {num }} / \omega_{p, b}$ & 0.3488 & 0.3318 & 0.2734 & 0.1953 & 0.08911 \\
\hline$\gamma_{\text {sim }} / \omega_{p, b}$ & 0.3459 & 0.3291 & 0.2722 & 0.1927 & 0.08745 \\
\hline$\frac{\gamma_{\text {sim }}-\gamma_{\text {num }}}{\gamma_{\text {num }}} \times 100 \%$ & $-0.81 \%$ & $-0.81 \%$ & $-0.44 \%$ & $-1.3 \%$ & $-1.9 \%$ \\
\hline
\end{tabular}

Table 1: Numerical solution of two-stream instability growth rate

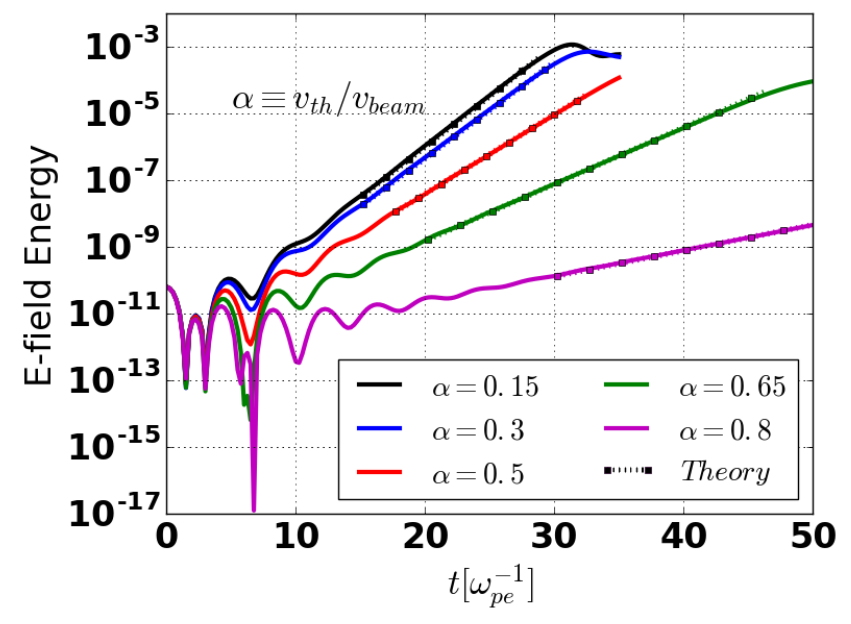

Figure 4: Growth of the electric field energy due to the two-stream instability for various ratios of the beam thermal speed $v_{t h, b}$ to the offset velocity $v_{b}$.

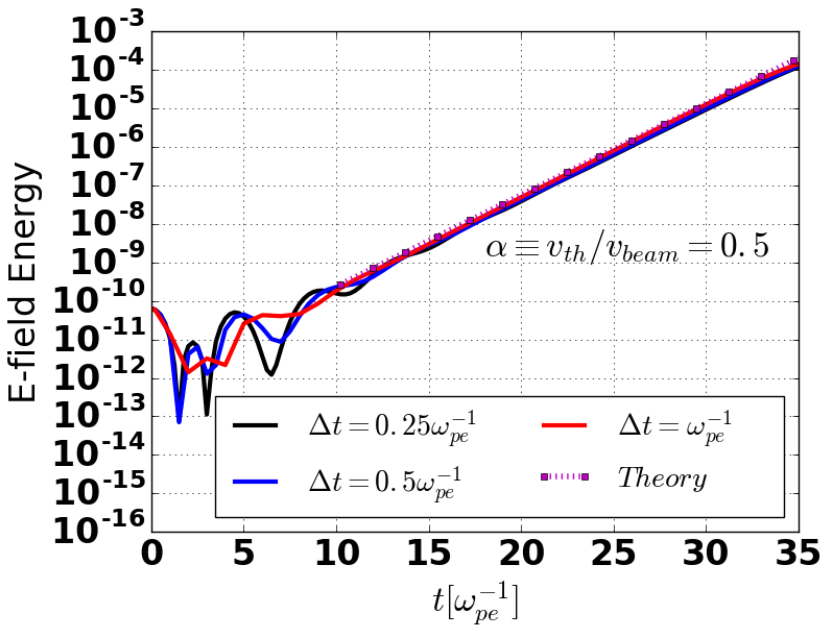

Figure 5: Growth of the electric field energy due to the two stream instability for various time-steps at a beam thermal velocity ratio of $v_{t h, b} / v_{b}=0.5$.

\subsection{Ion-acoustic shock wave}

The final test is the ion-acoustic shock wave (IASW) [50]. This problem is an excellent test of the scheme because it exhibits strongly nonlinear multi-scale behavior. In this problem, the dynamical time-scale of the system is orders of magnitude larger than the inverse electron plasma frequency, and so the simulation provides a stringent test of the HOLO algorithm to step over $\omega_{p e}^{-1}$ time-scales, which do not significantly contribute to the system evolution (since the evolution is largely ambipolar).

For this problem, we normalize particle mass to the proton mass, with the electron mass $m_{e}=1 / 1836$. We take the Debye length as $\lambda_{D}=1 / 36$, with the system length $L_{x}=144 \lambda_{D}$. The problem is initialized with sinusoidally perturbed ion and electron density profiles

$$
\begin{gathered}
n_{0, i}=1+0.2 \sin (k x), \\
n_{0, e}=1+0.2\left(1-k^{2} \lambda_{D}^{2}\right) \sin (k x),
\end{gathered}
$$

and with the same sinusoidal bulk velocity profiles for both species. The velocity is chosen such that the simulation proceeds in the frame of the shock:

$$
u_{\|, 0}=-1+0.2 \sin (k x) .
$$

The species temperatures are initially $T_{0, i}=0.05, T_{0, e}=1$, with the large temperature ratio chosen to avoid electron Landau damping [50]. As a consequence, the ion-acoustic time- and length-scales of the problem are much longer than the inverse plasma frequency $\omega_{p e}^{-1}$ and the Debye length [14]. The wave number is $k=2 \pi / L_{x}$. The simulation is performed with a velocity-space domain $c_{\|} \in[-8,+8], c_{\perp} \in[0,5]$, on a mesh of $N_{x}=128, N_{v_{\|}}=256$, $N_{v_{\perp}}=64$. The initial offset velocity of each species is set equal to the bulk velocity, $u_{\|, 0}^{*}=u_{\|, 0}$, with the initial normalizing speed equal to $v_{0, i}^{*}=\sqrt{2 T_{0, i}}, v_{0, e}^{*}=\sqrt{\frac{2 T_{0, e}}{m_{e}}}$. In this problem, the offset velocity is set to track the quantity $u_{\|, \alpha}+\Delta w_{\|, \alpha}$, where the normalized heat flux, $\Delta w_{\|, \alpha}=\frac{\left\langle\frac{1}{2} m_{\alpha}\left(v_{\|}-u_{\|}\right)(\boldsymbol{v}-\boldsymbol{u})^{2}, \tilde{f}_{\alpha}\right\rangle_{c}}{\frac{3}{2} n_{\alpha} T_{\alpha}}$, has been included to aid 
in capturing the significant non-Maxwellian wave-breaking feature in the ion distribution function at late times (see Fig. 6, lower right).

The first set of results consider a varying time-step size: $\Delta t=[1,10,100] \omega_{p e}^{-1}$. The timesteps were chosen in such a way that the smallest time-step is of the order of the stiff time-scale (i.e. the inverse plasma frequency) while the largest time-step is of the order of the dynamical time-scale (i.e., approximately the ion acoustic wave CFL: $100 \omega_{p e}^{-1} / \Delta t_{\mathrm{CFL}} \sim 2$ ). For this problem, a relative nonlinear convergence tolerance of $\epsilon_{r}=10^{-6}$ was used. At the largest $\Delta t$, we are stepping over many plasma periods. However, since this problem is not driven by the physics on this time-scale, we do not need to resolve it to capture the solution correctly. Figure 6 shows spatial profiles for
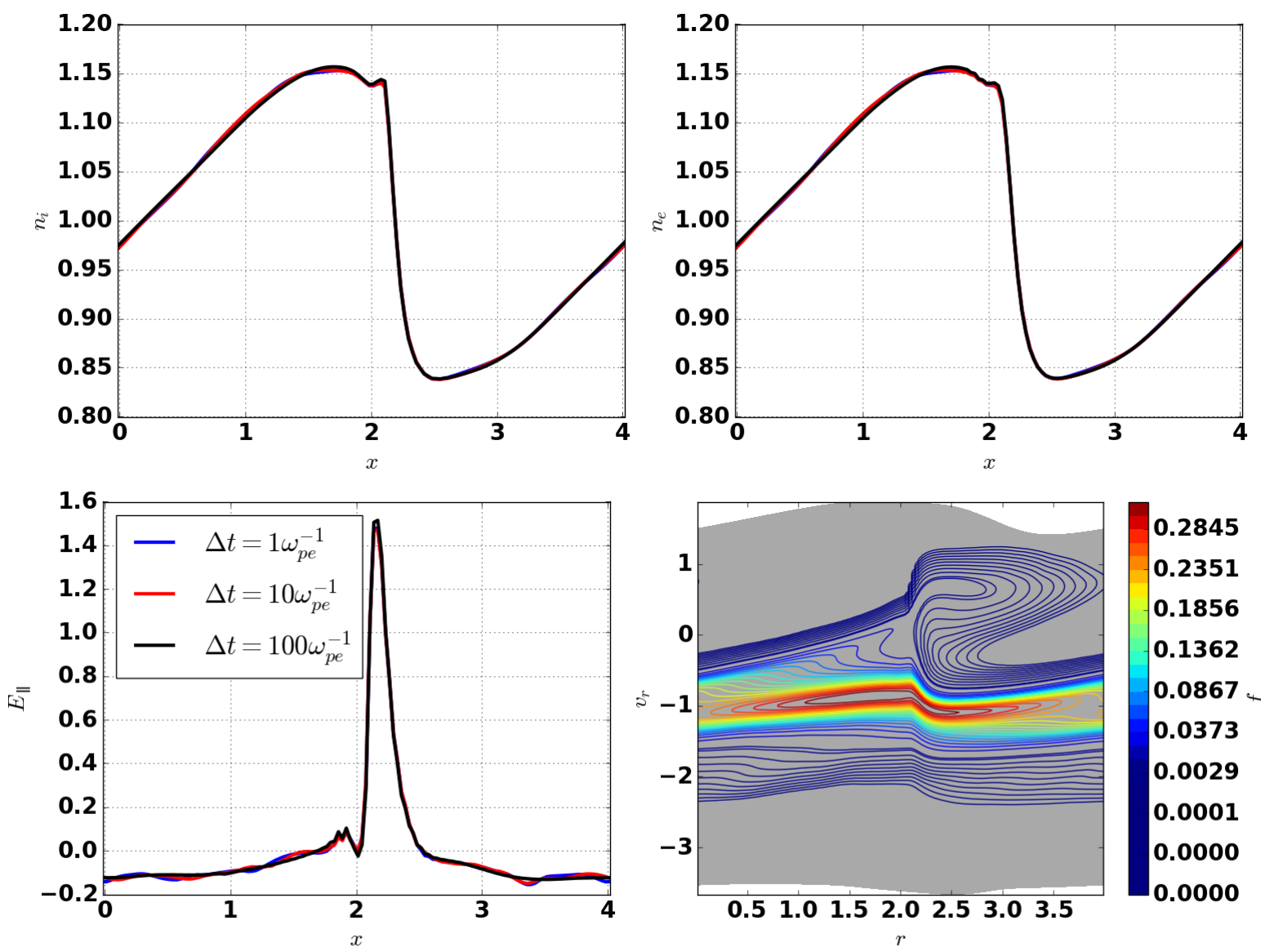

Figure 6: ion-acoustic shock wave solution for the ion number density (top left), electron number density (top right), and electric field (bottom left), at $t \approx 5000 \omega_{p e}^{-1}$ for various time-step sizes. Also given is a contour plot of the ion distribution, $f_{i}$, (bottom right) showing the wave-breaking in phase-space and the velocity-space domain adaptivity.

the number density of ions and electrons, and the electric field at $t \approx 5000 \omega_{p e}^{-1}$ for varying time-step sizes. We observe that the solution quality is not significantly affected even at time-steps far larger than the inverse electron plasma frequency (which is itself much larger than the explicit CFL). Table 2 shows the solver statistics for the simulations at each time-step size (obtained by averaging the number of HOLO iterations for each time-step over the simulation duration), indicating excellent performance even at large $\Delta t$. Here, we estimate the explicit timestep size as $\Delta t_{\text {explicit }}=\frac{\Delta x}{v_{\|, e, \max }}$. To demonstrate the capabilities of the velocity-space adaptive scheme, we also include in Fig. 6 a contour plot of the perpendicular velocity-integrated unnormalized ion distribution function, $f_{i, \|} \equiv$ $\left(v_{\alpha, i}^{*}\right)^{-1} \int_{v_{\perp, \min }}^{v_{\perp, \max }} \tilde{f}_{i}\left(x, c_{\|}, c_{\perp}\right) c_{\perp} d c_{\perp}$, at $t \approx 5000 \omega_{p e}^{-1}$ for the case with $\Delta t=\omega_{p e}^{-1}$. The velocity-space boundary adapts to variations in the ion bulk velocity, $u_{\|}$, and thermal speed, $v_{t h}=\sqrt{\frac{2 T}{m}}$, as can be clearly seen by the gray background fill of the simulation $\left(x, v_{\|}\right)$domain. We can also clearly see the characteristic 'wave-breaking' feature of the ion distribution in the velocity space. 


\begin{tabular}{|c|c|c|c|}
\hline$\Delta t / \omega_{p e}^{-1}$ & 1 & 10 & 100 \\
\hline \hline$\Delta t / \Delta t_{\text {explicit }}$ & $1.01 \mathrm{E}+1$ & $1.01 \mathrm{E}+2$ & $1.01 \mathrm{E}+3$ \\
\hline HOLO iters & 3.5 & 6.2 & 11.3 \\
\hline
\end{tabular}

Table 2: HOLO solver statistics for the ion-acoustic shock wave at various time-step sizes

In Sec. 4 , we discussed the need for enforcing discrete conservation properties. Figure 7 shows the error in mass, momentum, and energy conservation and Gauss's law for the IASW for various time-step sizes. The error is measured as the absolute value of the difference in a quantity at a given time relative to the initial value,

$$
\left|\frac{\Phi^{\mathrm{p}}-\Phi^{0}}{\Phi^{0}}\right|
$$

where $\Phi$ is the total mass TM, total momentum TP, or total energy TE. Additionally, we calculate the $L^{1}$ norm of the error in the discrete form of Gauss's law:

$$
|G L|^{p}=\sum_{i}^{N_{x}}\left|\epsilon_{0} \frac{\left(E_{\|, i+\frac{1}{2}}^{p}-E_{\|, i-\frac{1}{2}}^{p}\right)}{\Delta x_{i}}-\sum_{\alpha}^{N_{s p}} q_{\alpha} n_{\alpha, i}^{p}\right| .
$$

We see that, in all cases, the conservation error is affected by the different time-step sizes, but is kept well within acceptable levels.
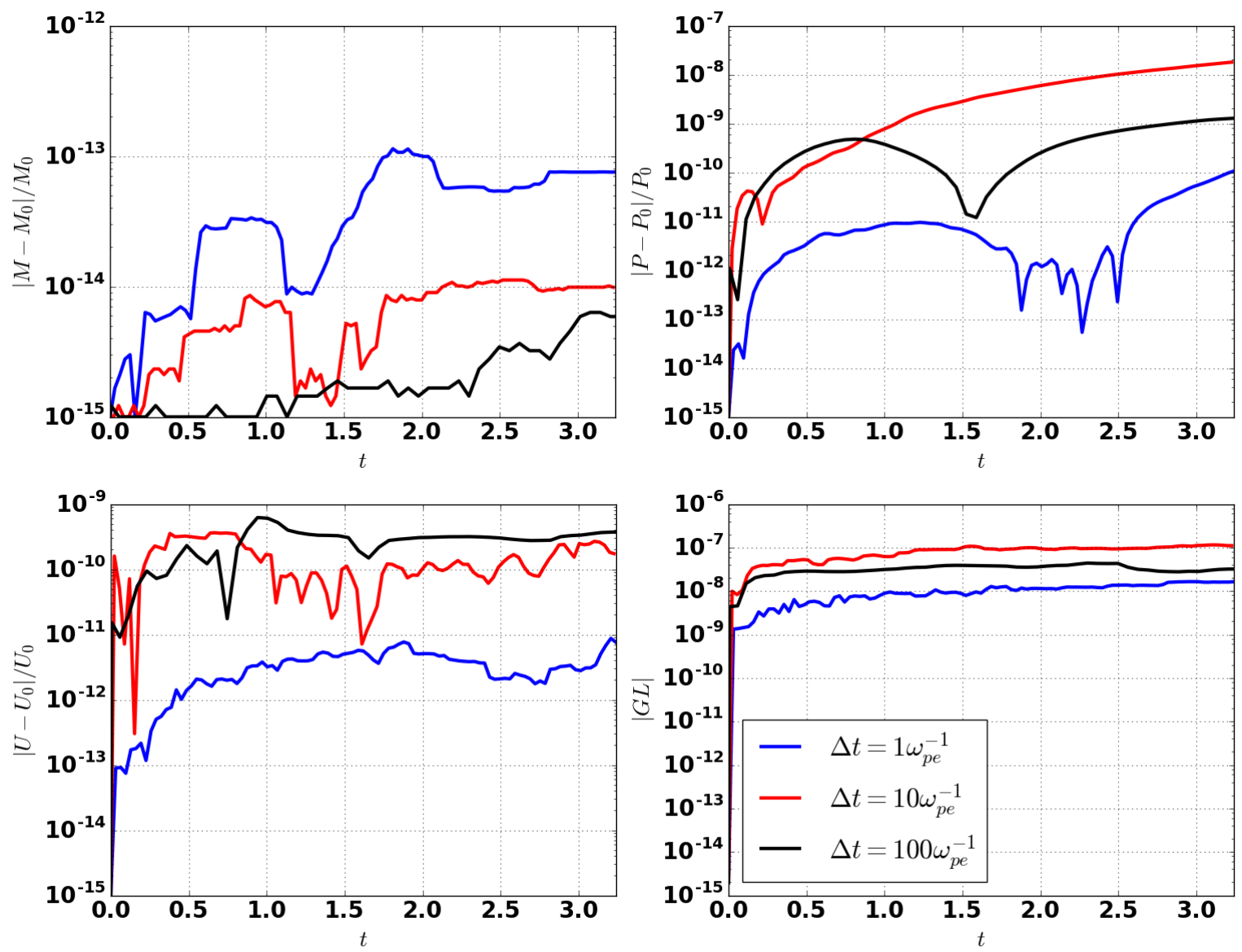

Figure 7: Conservation errors for the ion-acoustic shock wave simulations using various time-step sizes for the total mass (top left), total momentum (top right), total energy (bottom left), and charge from Gauss' law (bottom right). 
Next, we investigate the effects of the relative nonlinear convergence tolerance, $\epsilon_{r}$, on the magnitude of the conservation error. Here, we use a time-step of $\Delta t=\omega_{p e}^{-1}$, and vary the tolerance: $\epsilon_{r}=\left[10^{-4}, 10^{-6}, 10^{-8}\right]$. As we
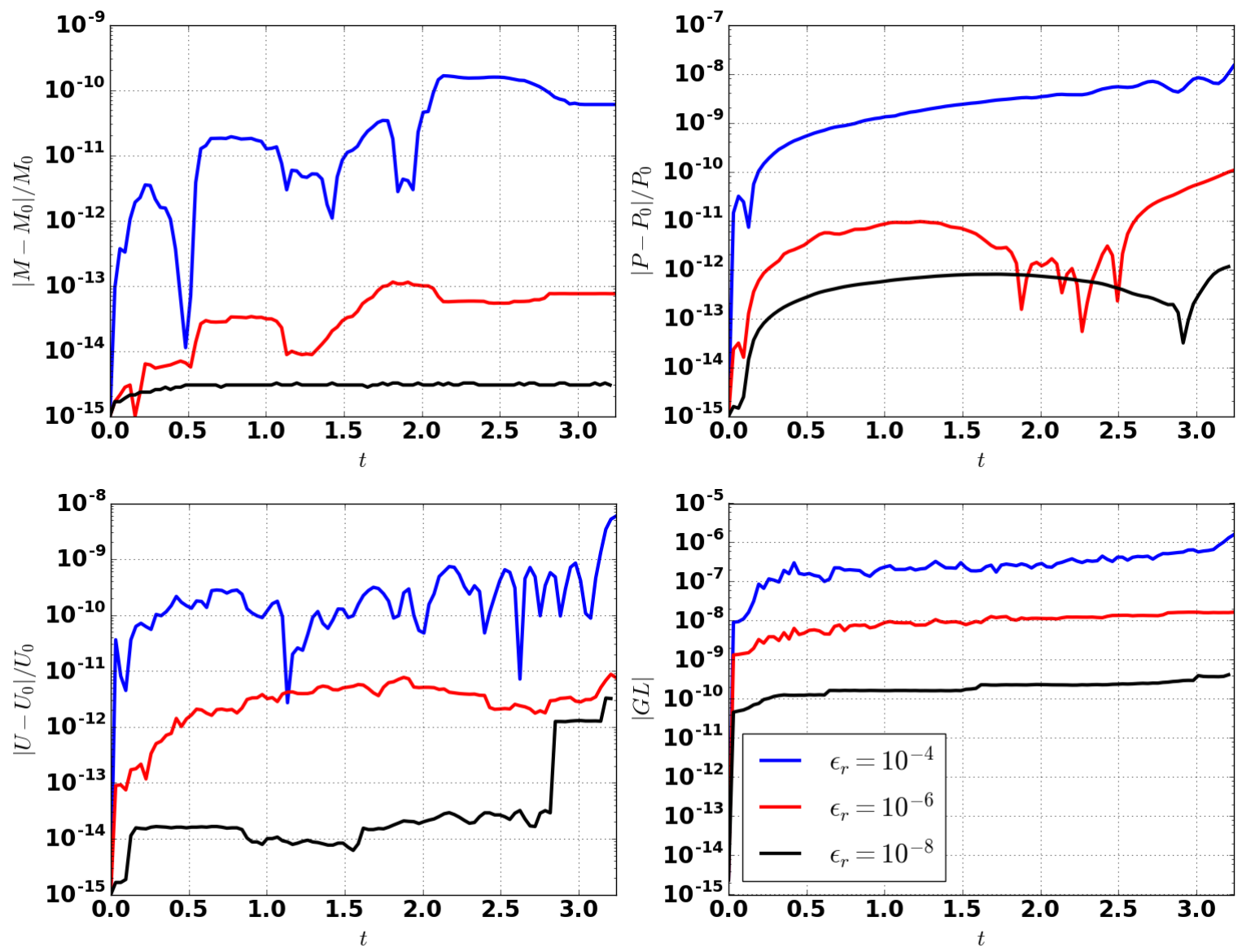

Figure 8: Conservation errors for the ion-acoustic shock wave simulations for various nonlinear convergence tolerances for the total mass (top left), total momentum (top right), total energy (bottom left), and charge from Gauss' law (bottom right).

can clearly see in Fig. 8, as the tolerance is tightened the conservation error decreases. Further decrease in $\epsilon_{r}$ will eventually push the error to machine roundoff.

\subsubsection{Importance of discrete conservation}

Here, we present IASW simulation results emphasizing the necessity of the developments presented in Sec.4. As we stated previously, in the Vlasov-Ampère system the most critical element of the discrete conservation strategy is ensuring that Gauss' law is satisfied. Here, we compare the case with $\Delta t=\omega_{p e}^{-1}, \epsilon_{r}=10^{-6}$ from Fig. 7 . with an identical case without the charge-conserving constraint function $\xi$ (and its associated pseudo-operator). The result is shown in Fig. 9. With all constraints except $\xi$ active, we see that the charge conservation error increases monotonically as expected. However, we also observe that the momentum and energy conservation errors experience a significant increase. The reason is that violations of charge conservation give rise to (significant) error in the electric field $E_{\|}$ [37], which results in unphysical acceleration in the Vlasov equation. This, in turn, results in violations of momentum and energy conservation. Figure 10 depicts the electric field $E_{\|}$at $t \approx 3100 \omega_{p e}^{-1}$. The $E_{\|}$for the simulation lacking charge conservation has at this stage accumulated significant error. Though not shown here, the solutions for other moment quantities (e.g., number density, temperature) also show significant errors.

We also investigate the effect of neglecting the momentum- and energy-conserving constraints (while maintaining the charge conserving constraint $\xi$ ). Here, we again compare the case with $\Delta t=\omega_{p e}^{-1}, \epsilon_{r}=10^{-6}$ from Fig. 7 to a case with the same parameters, but which only conserves charge (thereby failing to conserve momentum and energy). Figure 11 shows that indeed the simulation fails to conserve momentum and energy (albeit Gauss's law is still 

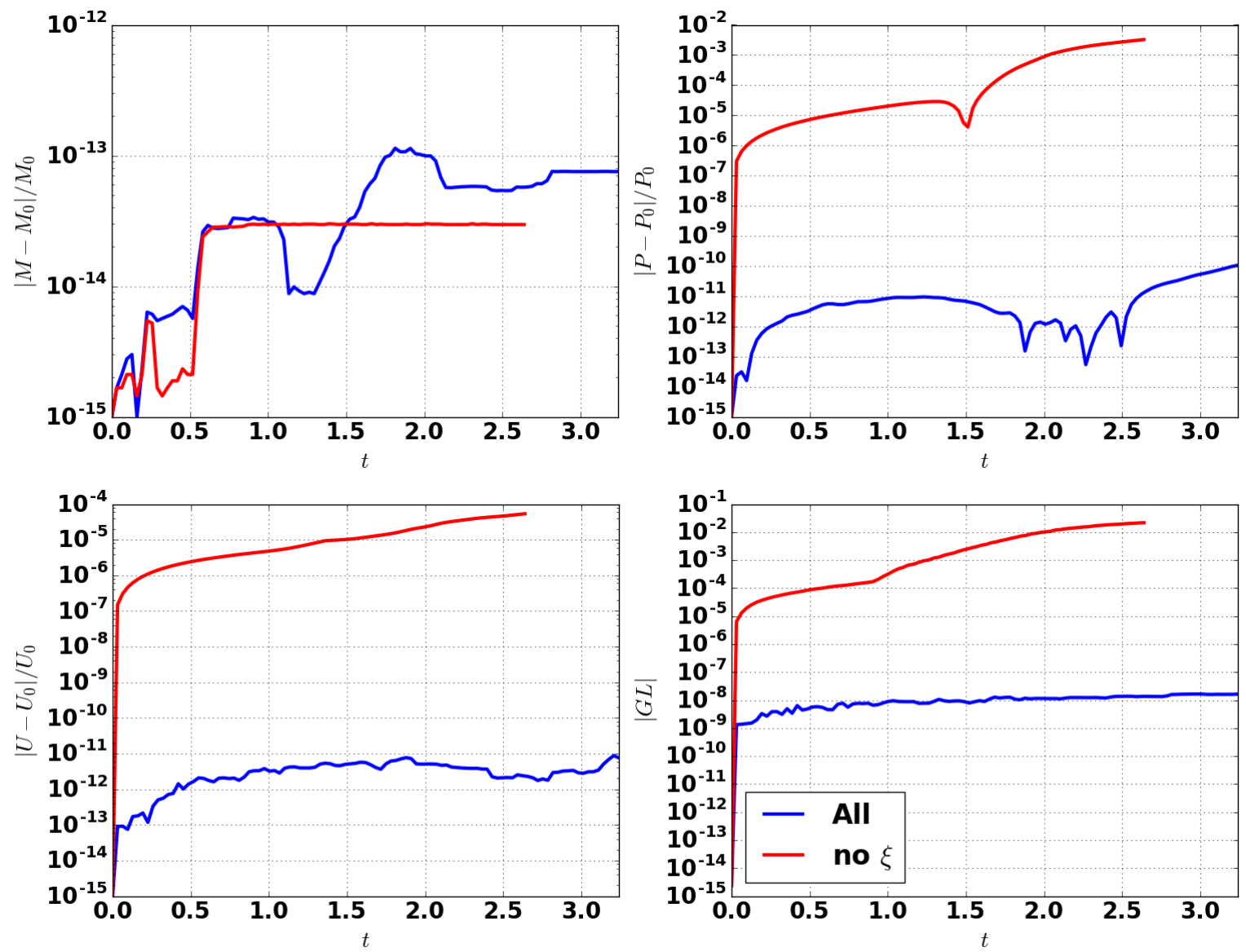

Figure 9: Conservation errors with and without charge conservation for the ion-acoustic shock wave simulations with for $\Delta t=\omega_{p e}^{-1}, \epsilon_{r}=10^{-6}$, comparing the solution with ("All", in blue) and without the charge-conserving nonlinear constraint function $\xi$ active ("no $\xi$ ", in red) for the total mass (top left), total momentum (top right), total energy (bottom left), and charge from Gauss' law (bottom right).

maintained), but without catastrophic failure. However, based on earlier studies [6], we do expect catastrophic failure when we couple our Vlasov solver with the Fokker-Planck collision operator.

\subsubsection{Importance of keeping the discrete averaged current in Ampère's equation}

In Sec. 2. we presented Ampère's equation with the spatially-averaged current, $\bar{j}_{\|}$, which is a necessary solvability constraint to preserve Galilean invariance in a periodic system. In this work, none of the problems presented possess an applied electric field, and thus the spatially averaged electric field, $\bar{E}_{\|}$, must be identically zero at all times. This is clearly seen from the relationship $\nabla_{\boldsymbol{x}} \Phi=-\boldsymbol{E}$, which relates the electric field to the electrostatic potential. However, as stated in Sec. 22 it is possible for a finite average current density $\bar{j}_{\|}$to exist even when $\bar{E}_{\|}=0$ due to nonlinear effects (see Appendix A. Thus, to ensure $\bar{E}_{\|}=0$, it is necessary to include $\bar{j}_{\|}$in Ampère's equation. In Fig. 12 we compare time traces of the spatially averaged electric field $\bar{E}_{\|}$,

$$
\bar{E}_{\|} \equiv \frac{1}{N_{x}} \sum_{i}^{N_{x}} E_{\|, i+\frac{1}{2}},
$$

for two simulations with and without $\bar{j}_{\|}$in Ampère's equation. All other parameters are identical to the $\Delta t=\omega_{p e}^{-1}$ case in Figs. 6 and 7 . We see that there is a significant error increase $\left[\mathcal{O}\left(10^{8}\right)\right]$ on the average electric field $\overline{E_{\|}}$when the average current is not included. 


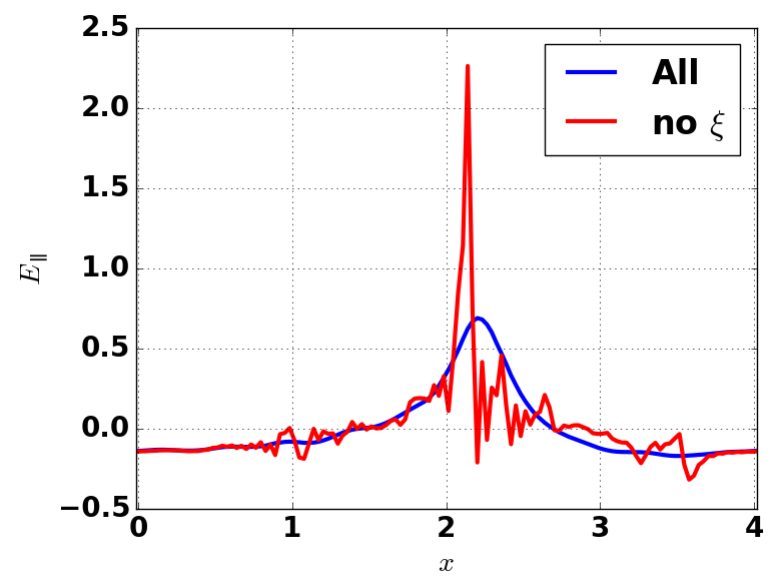

Figure 10: ion-acoustic shock wave solution for electric field $E_{\|}$, at $t \approx 3100 \omega_{p e}^{-1}$ for $\Delta t=\omega_{p e}^{-1}$ with ("All", in blue) and without the chargeconserving nonlinear constraint function $\xi$ active ("no $\xi$ ", in red).

\section{Conclusions}

We have presented a fully conserving, adaptive algorithm for numerically integrating the $1 \mathrm{D}-2 \mathrm{~V}$ multi-species Vlasov-Ampère system. The algorithm is applicable for the fully kinetic Vlasov system with an arbitrary number of species of arbitrary mass ratio. The velocity-space adaptivity scheme allows each species' velocity-space mesh to evolve according to variations in their bulk velocity and temperature resulting in very efficient velocity-space meshing. Conservation of the total mass, momentum, and energy, as well as Gauss's law, are enforced through the introduction of several nonlinear constraint functions, which eliminate the truncation error of the conservation properties. We emphasize that discrete conservation in our algorithm can be achieved with significant flexibility in temporal and spatial discretizations. The nonlinear scheme is efficiently accelerated via a HOLO algorithm, where a LO fluid representation is used to accelerated convergence of the HO kinetic system.

The present algorithm is tested with the linear and nonlinear Landau damping, as well as the two-stream instability and ion-acoustic shock wave. For the Landau damping and two-stream instability tests, we achieve excellent agreement with analytical and previously published growth/decay rates of the electric field energy. For the linear Landau damping test, we demonstrate that the algorithm achieves second-order convergence in time, configuration space, and velocity space. For the ion-acoustic shock wave test, we demonstrate that the algorithm remains stable when taking timesteps much larger than stiff time-scales (such as the inverse plasma frequency $\omega_{p e}^{-1}$ ) without affecting quality of the solution and while maintaining discrete conservation. We also demonstrate a commensurate decrease in the discrete conservation error with decreasing nonlinear convergence tolerance, in principle allowing us to drive the error to machine precision if desired. Further, we demonstrate that without enforcing discrete conservation (particularly for charge conservation), the solution can degrade significantly.

\section{Acknowledgments}

This work was supported by the Thermonuclear Burn Initiative of the Advanced Simulation and Computing Program, used resources provided by the Institutional Computing Program at Los Alamos National Laboratory, and was performed under the auspices of the National Nuclear Security Administration of the U.S. Department of Energy at Los Alamos National Laboratory, managed by Triad National Security, LLC under contract 89233218 CNA000001.

\section{Appendix A. Nonlinear generation of finite average current density}

As presented in Sec. 2, in one-dimensional configuration space the Vlasov-Ampère system of equations becomes

$$
\begin{array}{r}
\partial_{t} f_{\alpha}+\partial_{x}\left(v_{\|} f_{\alpha}\right)+\frac{q_{\alpha}}{m_{\alpha}} E_{\|} \partial_{v_{\|}}\left(f_{\alpha}\right)=0, \\
\epsilon_{0} \partial_{t} E_{\|}+\sum_{\alpha} q_{\alpha} \Gamma_{\|, \alpha}=\bar{j}_{\|} .
\end{array}
$$



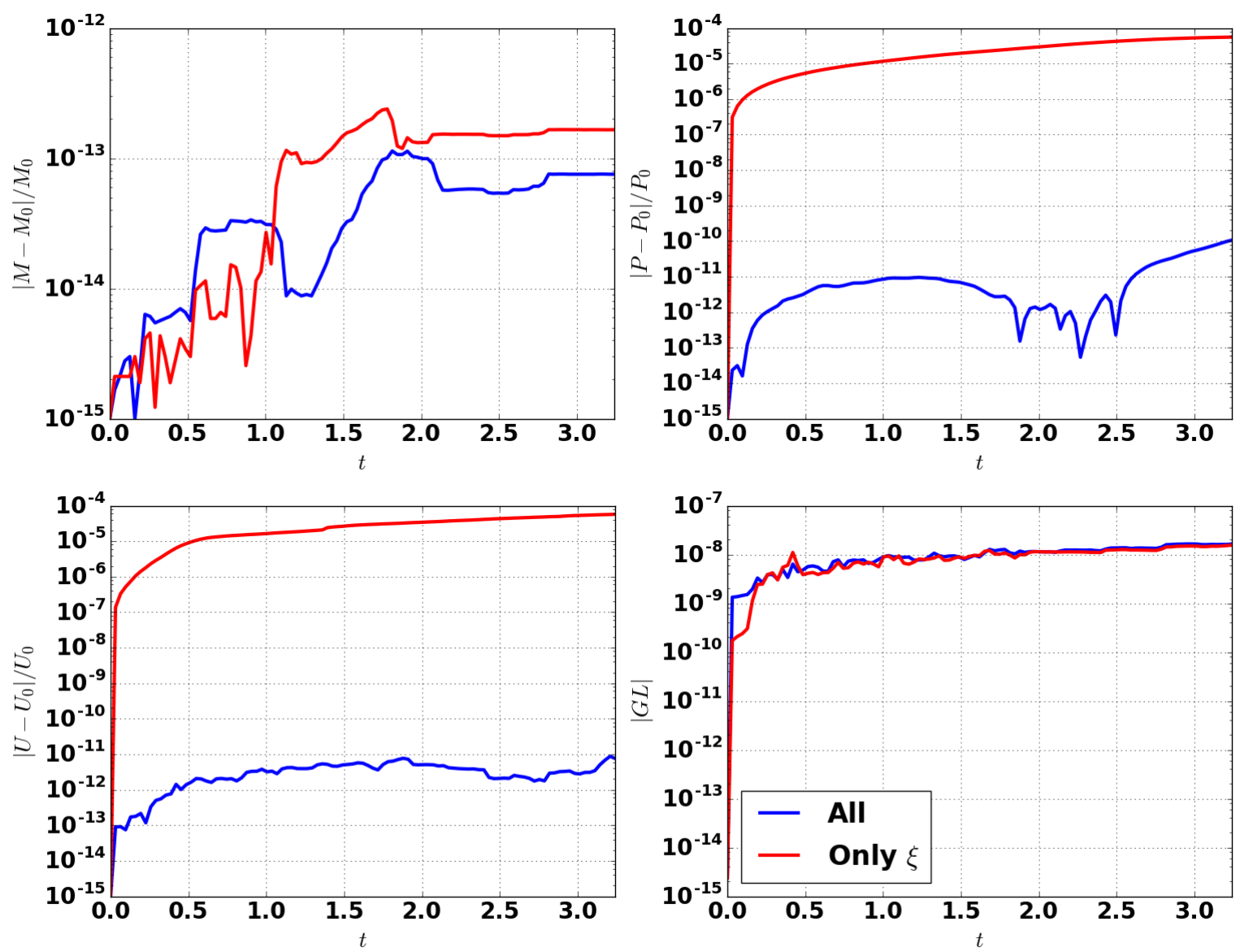

Figure 11: Conservation errors for the ion-acoustic shock wave with all nonlinear constraint functions active ("All", in blue) and with only the charge-conserving constraint function $\xi$ active ("Only $\xi$ ", in red). The total mass (top left), total momentum (top right), total energy (bottom left), and charge from Gauss' law (bottom right) are depicted.

We note again the presence of the average current density $\bar{j}_{\|}$, which is necessary in 1D periodic systems. Here $\bar{j}_{\|}$is taken to be the average of the time-dependent current density, $\bar{j}_{\|}(t)$, rather than the initial current density, $\bar{j}_{\|}(t=0)$. This is because a finite average current density may be generated due to nonlinear effects even in systems where the electric field is a gradient (i.e. $\bar{E}_{\|}=0$ ). This may be demonstrated as follows.

The $v_{\|}^{1}$ moment of Eq. 6 will produce the governing equation for particle flux density $\Gamma_{\|, \alpha}$

$$
\partial_{t} \Gamma_{\|, \alpha}+\partial_{x} S_{\|\|, \alpha}^{(2)}-\frac{q_{\alpha}}{m_{\alpha}} n_{\alpha} E_{\|}=0
$$

Recalling the definition of current density, $j_{\|}=\sum_{\alpha} q_{\alpha} \Gamma_{\|, \alpha}$, A.1 becomes the equation for current conservation,

$$
\partial_{t} j_{\|}+\partial_{x}\left(\sum_{\alpha} q_{\alpha} S_{\|\|, \alpha}^{(2)}\right)-\sum_{\alpha} \frac{q_{\alpha}^{2}}{m_{\alpha}} n_{\alpha} E_{\|}=0 .
$$

Taking the spatial average of Eq. A.2, we obtain

$$
\partial_{t} \bar{j}_{\|}-\sum_{\alpha} \frac{q_{\alpha}^{2}}{m_{\alpha}} \overline{\left(n_{\alpha} E_{\|}\right)}=0 .
$$

If we make a substitution for $n_{\alpha}=n_{\alpha}^{*}+\bar{n}_{\alpha}$, where $\bar{n}_{\alpha}$ is the spatial average of $n_{\alpha}$ (i.e., a constant) and $n_{\alpha}^{*}$ is spatially varying (with $\overline{n_{\alpha}^{*}}=0$ ), we see that Eq. A.3 becomes

$$
\partial_{t} \bar{j}_{\|}=\sum_{\alpha} \frac{q_{\alpha}^{2}}{m_{\alpha}}\left[\overline{\left(n_{\alpha}^{*} E_{\|}\right)}\right],
$$




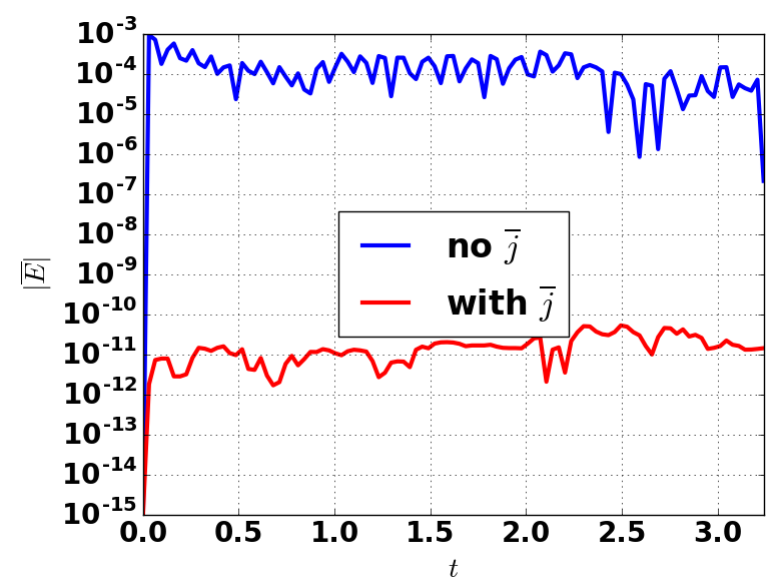

Figure 12: The spatially-averaged electric field $\bar{E}_{\|}$vs. time with ("with $\bar{j}$ " red) and without ("no $\bar{j}$ ", in blue) the spatially-averaged current $\bar{j}_{\|}$ included in Ampère's equation.

which is in general non-zero, even though $\bar{n}_{\alpha}^{*}=0$ and $\bar{E}_{\|}=0$.

\section{Appendix B. Derivation of continuum symmetries for conservation}

Appendix B.1. Mass \& charge conservation

Mass conservation is demonstrated by taking the $m_{\alpha} v^{0}$ moment of Eq. 10 :

$$
\begin{aligned}
\left\langle m_{\alpha}, \partial_{t} \tilde{f}_{\alpha}\right\rangle_{\boldsymbol{c}}+\left\langle m_{\alpha}, \partial_{x}\left(v_{\alpha}^{*} \hat{v}_{\|} \tilde{f}_{\alpha}\right)\right\rangle_{\boldsymbol{c}}+\frac{q_{\alpha}}{m_{\alpha} v_{\alpha}^{*}} E_{\|}\left\langle m_{\alpha}, \partial_{c_{\|}} \tilde{f}_{\alpha}\right\rangle_{\boldsymbol{c}} & \\
& -\frac{1}{v_{\alpha}^{*}}\left\langle m_{\alpha}, \nabla_{\boldsymbol{c}} \cdot\left\{\left[\partial_{t}(\boldsymbol{v})+\partial_{x}(\boldsymbol{v}) \hat{v}_{\|} v_{\alpha}^{*}\right] \tilde{f}_{\alpha}\right\}\right\rangle_{\boldsymbol{c}}=0 .
\end{aligned}
$$

Observing that the first two terms produce the mass density $\left\langle m_{\alpha}, \tilde{f}_{\alpha}\right\rangle_{c}=m_{\alpha} n_{\alpha}$ and the parallel momentum density $\left\langle m_{\alpha}, v_{\alpha}^{*} \hat{v}_{\|} \tilde{f}_{\alpha}\right\rangle_{c}=m_{\alpha} \Gamma_{\|, \alpha}$ while the last two terms are zeros, we obtain the mass conservation equation,

$$
m_{\alpha}\left[\partial_{t} n_{\alpha}+\partial_{x} \Gamma_{\|, \alpha}\right]=0
$$

This is straightforward to satisfy in the discrete with appropriate boundary conditions on the distribution $f_{\alpha}$.

However, we must also be cognizant of the symmetry between Ampère's and Gauss's laws and the above statement of mass conservation. If we take Eq. (5) in one dimension we find

$$
\partial_{t}\left(\sum_{\alpha} \rho_{q, \alpha}\right)+\partial_{x}\left(\sum_{\alpha} j_{\|, \alpha}\right)=0,
$$

where $\rho_{q, \alpha} \equiv q_{\alpha} n_{\alpha}$ and $j_{\|, \alpha} \equiv q_{\alpha} \Gamma_{\|, \alpha}$. Of course, underlying Eq. [B.3 is simply the summation over all species of Eq. (B.2):

$$
\partial_{t}\left(\sum_{\alpha} \rho_{q, \alpha}\right)+\partial_{x}\left(\sum_{\alpha} j_{\|, \alpha}\right)=\sum_{\alpha} q_{\alpha}\left(\partial_{t} n_{\alpha}+\partial_{x} \Gamma_{\|, \alpha}\right) .
$$

Thus, we see that the charge density in Gauss's law and the current in Ampère's equation must be proportional to the particle number density and flux in Eq. (B.2). 
Appendix B.2. Momentum conservation

Momentum conservation is demonstrated by taking the $m_{\alpha} v_{\|}$moment of Eq. [10]:

$$
\begin{aligned}
\left\langle m_{\alpha} v_{\|}, \partial_{t} \tilde{f}_{\alpha}\right\rangle_{\boldsymbol{c}}+\left\langle m_{\alpha} v_{\|}, \partial_{x}\left(v_{\alpha}^{*} \hat{v}_{\|} \tilde{f}_{\alpha}\right)\right\rangle_{\boldsymbol{c}}+\frac{q_{\alpha}}{m_{\alpha} v_{\alpha}^{*}} E_{\|}\left\langle m_{\alpha} v_{\|}, \partial_{c_{\|}} \tilde{f}_{\alpha}\right\rangle_{\boldsymbol{c}} & \\
\left.-\frac{1}{v_{\alpha}^{*}}\left\langle m_{\alpha} v_{\|}, \nabla_{\boldsymbol{c}} \cdot\left\{\left[\partial_{t}(\boldsymbol{v})\right]+\partial_{x}(\boldsymbol{v}) \hat{v}_{\|} v_{\alpha}^{*}\right] \tilde{f}_{\alpha}\right\}\right\rangle_{\boldsymbol{c}} & =0 .
\end{aligned}
$$

First, we take note of the terms involving temporal derivatives (boxed in blue in Eq. (B.5), and apply the chain rule with integration by parts (once again applying $\lim _{c \rightarrow \pm \infty} f_{\alpha}=0$ ):

$$
\begin{array}{r}
\left\langle m_{\alpha} v_{\|}, \partial_{t} \tilde{f}_{\alpha}\right\rangle_{\boldsymbol{c}}-\frac{1}{v_{\alpha}^{*}}\left\langle m_{\alpha} v_{\|}, \nabla_{\boldsymbol{c}} \cdot\left\{\partial_{t}(\boldsymbol{v}) \tilde{f}_{\alpha}\right\}\right\rangle_{\boldsymbol{c}}=\left\langle 1, \partial_{t}\left(m_{\alpha} v_{\|} \tilde{f}_{\alpha}\right)\right\rangle_{\boldsymbol{c}}-\left\langle m_{\alpha} \tilde{f}_{\alpha}, \partial_{t} v_{\|}\right\rangle_{\boldsymbol{c}} \\
+\frac{1}{v_{\alpha}^{*}}\left\langle 1, m_{\alpha} \partial_{t}(\boldsymbol{v}) \tilde{f}_{\alpha} \cdot \nabla_{\boldsymbol{c}} v_{\|}\right\rangle_{\boldsymbol{c}} .
\end{array}
$$

In a similar manner, we may inspect the terms in Eq. (B.5) involving spatial derivatives (boxed in red)

$$
\begin{aligned}
\left\langle m_{\alpha} v_{\|}, \partial_{x}\left(v_{\alpha}^{*} \hat{v}_{\|} \tilde{f}_{\alpha}\right)\right\rangle_{\boldsymbol{c}}-\frac{1}{v_{\alpha}^{*}}\left\langle m_{\alpha} v_{\|}, \nabla_{\boldsymbol{c}} \cdot\left\{\partial_{x}(\boldsymbol{v}) \hat{v}_{\|} v_{\alpha}^{*} \tilde{f}_{\alpha}\right\}\right\rangle_{\boldsymbol{c}} & =\left\langle 1, \partial_{x}\left(m_{\alpha} v_{\|}^{2} \tilde{f}_{\alpha}\right)\right\rangle_{\boldsymbol{c}}-\left\langle m_{\alpha} v_{\|} \tilde{f}_{\alpha}, \partial_{x} v_{\|}\right\rangle_{\boldsymbol{c}} \\
& +\frac{1}{v_{\alpha}^{*}}\left\langle 1, m_{\alpha} \partial_{x}(\boldsymbol{v}) \hat{v}_{\|} v_{\alpha}^{*} \tilde{f}_{\alpha} \cdot \nabla_{\boldsymbol{c}} v_{\|}\right\rangle_{\boldsymbol{c}} \cdot \quad \text { (B.7) }
\end{aligned}
$$

Observing that $\nabla_{c} v_{\|}=v_{\alpha}^{*} e_{\|}$, we see that the last two terms cancel in both Eq. (B.6) and Eq. (B.7). Together these equations become

$$
\begin{aligned}
\left\langle m_{\alpha} v_{\|}, \partial_{t} \tilde{f}_{\alpha}\right\rangle_{\boldsymbol{c}}+\left\langle m_{\alpha} v_{\|}, \partial_{x}\left(v_{\alpha}^{*} \hat{v}_{\|} \tilde{f}_{\alpha}\right)\right\rangle_{\boldsymbol{c}}-\frac{1}{v_{\alpha}^{*}}\left\langle m_{\alpha} v_{\|}, \nabla_{\boldsymbol{c}} \cdot\left\{\left[\partial_{t}(\boldsymbol{v})+\partial_{x}(\boldsymbol{v}) \hat{v}_{\|} v_{\alpha}^{*}\right] \tilde{f}_{\alpha}\right\}\right\rangle_{\boldsymbol{c}} \\
=\left\langle 1, \partial_{t}\left(m_{\alpha} v_{\|} \tilde{f}_{\alpha}\right)\right\rangle_{\boldsymbol{c}}+\left\langle 1, \partial_{x}\left(m_{\alpha} v_{\|}^{2} \tilde{f}_{\alpha}\right)\right\rangle_{\boldsymbol{c}}
\end{aligned}
$$

If we inspect the acceleration term in Eq. $(\overline{\mathrm{B} .5})$ and observe that $\partial_{c_{\|}} v_{\|}=v_{\alpha}^{*}$, we find

$$
\frac{q_{\alpha}}{m_{\alpha} v_{\alpha}^{*}} E_{\|}\left\langle m_{\alpha} v_{\|}, \partial_{c_{\|}} \tilde{f}_{\alpha}\right\rangle_{\boldsymbol{c}}=-\frac{q_{\alpha}}{m_{\alpha} v_{\alpha}^{*}} E_{\|}\left\langle m_{\alpha} \tilde{f}_{\alpha}, \partial_{c_{\|}} v_{\|}\right\rangle_{\boldsymbol{c}}=-\frac{q_{\alpha}}{m_{\alpha}} E_{\|}\left\langle 1, m_{\alpha} \tilde{f}_{\alpha}\right\rangle_{\boldsymbol{c}} .
$$

Thus, if we sum over all species, Eq. (B.5) becomes

$$
\sum_{\alpha}\left[\left\langle 1, \partial_{t}\left(m_{\alpha} v_{\|} \tilde{f}_{\alpha}\right)\right\rangle_{\boldsymbol{c}}+\left\langle 1, \partial_{x}\left(m_{\alpha} v_{\|}^{2} \tilde{f}_{\alpha}\right)\right\rangle_{\boldsymbol{c}}-\frac{q_{\alpha}}{m_{\alpha}} E_{\|}\left\langle 1, m_{\alpha} \tilde{f}_{\alpha}\right\rangle_{\boldsymbol{c}}\right]=0
$$

We may now define $\left\langle 1, m_{\alpha} v_{\|}^{2} \tilde{f}_{\alpha}\right\rangle_{c}=S_{2,\|\|, \alpha}$. If we recall Gauss's law, Eq. 3 , we may make a substitution in the acceleration term:

$$
\partial_{t} P_{\|}+\partial_{x} S_{2,\|\|}-E_{\|} \epsilon_{0} \partial_{x} E_{\|}=\partial_{t} P_{\|}+\partial_{x}\left[S_{2,\|\|}-\frac{1}{2} \epsilon_{0} E_{\|}^{2}\right]=0
$$

where $P_{\|}=\sum_{\alpha} m_{\alpha} \Gamma_{\|, \alpha}$ is the total (parallel) momentum density, $S_{2,\|\|}$ is the total (fluid) stress and $\frac{1}{2} \epsilon_{0} E_{\|}^{2}$ is the electrostatic stress. Equation (B.11) is a succinct statement of total momentum conservation - when we integrate over a periodic domain $\frac{1}{L} \int_{0}^{L} d x$ to obtain the total system momentum we obtain

$$
\partial_{t} \bar{P}_{\|}=0
$$

where $\bar{P}_{\|}=\frac{1}{L} \int_{0}^{L} P_{\|} d x$. The key symmetries here are 1) the equivalences in Eqs. B.6. and (B.7), and 2) the equivalence in Eq. (B.9) of the density arising from the acceleration term to that which appears in the temporal term - i.e., through Gauss's law in Eq. B.3. In general, none of these symmetries are guaranteed in the discrete system. Indeed, we see that the second symmetry here involving Gauss's law and the acceleration operator may directly contradict the charge-conservation requirement leading to Eq. B.3. 


\section{Appendix B.3. Energy conservation}

Energy conservation is demonstrated by taking the $m_{\alpha} \frac{v^{2}}{2}$ moment of Eq. 10 :

$$
\begin{aligned}
\left\langle m_{\alpha} \frac{1}{2} \boldsymbol{v}^{2}, \partial_{t} \tilde{f}_{\alpha}\right\rangle_{\boldsymbol{c}}+\left\langle m_{\alpha} \frac{1}{2} \boldsymbol{v}^{2}, \partial_{x}\left(v_{\alpha}^{*} \hat{v}_{\|} \tilde{f}_{\alpha}\right)\right\rangle_{\boldsymbol{c}} & +\frac{q_{\alpha}}{m_{\alpha} v_{\alpha}^{*}} E_{\|}\left\langle m_{\alpha} \frac{1}{2} \boldsymbol{v}^{2}, \partial_{c_{\|}} \tilde{f}_{\alpha}\right\rangle_{\boldsymbol{c}} \\
& -\frac{1}{v_{\alpha}^{*}}\left\langle m_{\alpha} \frac{1}{2} \boldsymbol{v}^{2}, \nabla_{\boldsymbol{c}} \cdot\left\{\left[\partial_{t}(\boldsymbol{v})+\partial_{x}(\boldsymbol{v}) \hat{v}_{\|} v_{\alpha}^{*}\right] \tilde{f}_{\alpha}\right\}\right\rangle_{\boldsymbol{c}}=0 .
\end{aligned}
$$

As with momentum conservation, we investigate the terms with temporal and spatial derivatives separately in order to expose their respective conservation symmetries. Integrating by parts and assuming no boundary contributions gives, for the temporal terms:

$$
\begin{aligned}
& \left\langle m_{\alpha} \frac{1}{2} \boldsymbol{v}^{2}, \partial_{t} \tilde{f}_{\alpha}\right\rangle_{\boldsymbol{c}}-\frac{1}{v_{\alpha}^{*}}\left\langle m_{\alpha} \frac{1}{2} \boldsymbol{v}^{2}, \nabla_{\boldsymbol{c}} \cdot\left\{\partial_{t}(\boldsymbol{v}) \tilde{f}_{\alpha}\right\}\right\rangle_{\boldsymbol{c}}= \\
& \left\langle 1, \partial_{t}\left(m_{\alpha} \frac{1}{2} \boldsymbol{v}^{2} \tilde{f}_{\alpha}\right)\right\rangle_{\boldsymbol{c}}-\left\langle m_{\alpha} \tilde{f}_{\alpha}, \partial_{t}\left(\frac{1}{2} \boldsymbol{v}^{2}\right)\right\rangle_{\boldsymbol{c}}+\frac{1}{v_{\alpha}^{*}}\left\langle 1, m_{\alpha} \partial_{t}(\boldsymbol{v}) \tilde{f}_{\alpha} \cdot \nabla_{\boldsymbol{c}}\left(\frac{1}{2} \boldsymbol{v}^{2}\right)\right\rangle_{\boldsymbol{c}},
\end{aligned}
$$

and for the spatial terms:

$$
\begin{aligned}
& \left\langle m_{\alpha} \frac{1}{2} \boldsymbol{v}^{2}, \partial_{x}\left(v_{\|} \tilde{f}_{\alpha}\right)\right\rangle_{\boldsymbol{c}}-\frac{1}{v_{\alpha}^{*}}\left\langle m_{\alpha} \frac{1}{2} \boldsymbol{v}^{2}, \nabla_{\boldsymbol{c}} \cdot\left\{\partial_{x}(\boldsymbol{v}) v_{\|} \tilde{f}_{\alpha}\right\}\right\rangle_{\boldsymbol{c}}= \\
& \quad\left\langle 1, \partial_{x}\left(m_{\alpha} \frac{1}{2} \boldsymbol{v}^{2} v_{\|} \tilde{f}_{\alpha}\right)\right\rangle_{\boldsymbol{c}}-\left\langle m_{\alpha} v_{\|} \tilde{f}_{\alpha}, \partial_{x}\left(\frac{1}{2} \boldsymbol{v}^{2}\right)\right\rangle_{\boldsymbol{c}}+\frac{1}{v_{\alpha}^{*}}\left\langle 1, m_{\alpha} \partial_{x}(\boldsymbol{v}) v_{\|} \tilde{f}_{\alpha} \cdot \nabla_{\boldsymbol{c}}\left(\frac{1}{2} \boldsymbol{v}^{2}\right)\right\rangle_{\boldsymbol{c}} .
\end{aligned}
$$

Here, we observe that $\nabla_{\boldsymbol{c}}\left(\frac{1}{2} \boldsymbol{v}^{2}\right)=\boldsymbol{v} v_{\alpha}^{*}, \partial_{x}\left(\frac{1}{2} \boldsymbol{v}^{2}\right)=\boldsymbol{v} \cdot \partial_{x} \boldsymbol{v}$, and $\partial_{t}\left(\frac{1}{2} \boldsymbol{v}^{2}\right)=\boldsymbol{v} \cdot \partial_{t} \boldsymbol{v}$. Thus, the last two terms on the right-hand sides of Eqs. (B.13) and (B.14) cancel, and together these equations become

$$
\begin{aligned}
\left\langle m_{\alpha} \frac{1}{2} \boldsymbol{v}^{2}, \partial_{t} \tilde{f}_{\alpha}\right\rangle_{\boldsymbol{c}}+\left\langle m_{\alpha} \frac{1}{2} \boldsymbol{v}^{2}, \partial_{x}\left(v_{\|} \tilde{f}_{\alpha}\right)\right\rangle_{\boldsymbol{c}} & -\frac{1}{v_{\alpha}^{*}}\left\langle m_{\alpha} \frac{1}{2} \boldsymbol{v}^{2}, \nabla_{\boldsymbol{c}} \cdot\left\{\left[\partial_{t}(\boldsymbol{v})+\partial_{x}(\boldsymbol{v}) v_{\|}\right] \tilde{f}_{\alpha}\right\}\right\rangle_{\boldsymbol{c}} \\
& =\left\langle 1, \partial_{t}\left(m_{\alpha} \frac{1}{2} \boldsymbol{v}^{2} \tilde{f}_{\alpha}\right)\right\rangle_{\boldsymbol{c}}+\left\langle 1, \partial_{x}\left(m_{\alpha} \frac{1}{2} \boldsymbol{v}^{2} v_{\|} \tilde{f}_{\alpha}\right)\right\rangle_{\boldsymbol{c}} .
\end{aligned}
$$

Returning to the acceleration term in Eq. $B .12$ and integrating by parts gives

$$
\frac{q_{\alpha}}{m_{\alpha} v_{\alpha}^{*}} E_{\|}\left\langle m_{\alpha} \frac{1}{2} \boldsymbol{v}^{2}, \partial_{c_{\|}} \tilde{f}_{\alpha}\right\rangle_{\boldsymbol{c}}=-\frac{q_{\alpha}}{m_{\alpha} v_{\alpha}^{*}} E_{\|}\left\langle m_{\alpha} \tilde{f}_{\alpha}, \partial_{c_{\|}}\left(\frac{1}{2} \boldsymbol{v}^{2}\right)\right\rangle_{\boldsymbol{c}}=-\frac{q_{\alpha}}{m_{\alpha}} E_{\|}\left\langle 1, m_{\alpha} v_{\|} \tilde{f}_{\alpha}\right\rangle_{\boldsymbol{c}}
$$

where we utilized the relationship $\partial_{c_{\|}}\left(\frac{1}{2} \boldsymbol{v}^{2}\right)=v_{\|} v_{\alpha}^{*}$.

Combining the preceding results and summing over all species $\alpha$, Eq. (B.12] becomes

$$
\sum_{\alpha}\left[\left\langle 1, \partial_{t}\left(m_{\alpha} \frac{1}{2} \boldsymbol{v}^{2} \tilde{f}_{\alpha}\right)\right\rangle_{\boldsymbol{c}}+\left\langle 1, \partial_{x} m_{\alpha} \frac{1}{2} \boldsymbol{v}^{2} v_{\|} \tilde{f}_{\alpha}\right\rangle_{\boldsymbol{c}}-\frac{q_{\alpha}}{m_{\alpha}} E_{\|}\left\langle 1, m_{\alpha} v_{\|} \tilde{f}_{\alpha}\right\rangle_{\boldsymbol{c}}\right]=0 .
$$

We note the definitions $\left\langle 1, m_{\alpha} \frac{1}{2} \boldsymbol{v}^{2} \tilde{f}_{\alpha}\right\rangle_{\boldsymbol{c}}=\varepsilon_{\alpha}$ and $\left\langle 1, m_{\alpha} \frac{1}{2} \boldsymbol{v}^{2} v_{\|} \tilde{f}_{\alpha}\right\rangle_{\boldsymbol{c}}=S_{3, \|, \alpha}$ and, recalling Ampère's equation, we introduce it in the acceleration term to find

$$
\partial_{t} U+\partial_{x} S_{3, \|}+E_{\|} \epsilon_{0} \partial_{t} E_{\|}=\partial_{t}\left[U+\frac{1}{2} \epsilon_{0} E_{\|}^{2}\right]+\partial_{x} S_{3, \|}-E_{\|} \bar{j}_{\|}=0 .
$$

Here, $U$ is the total (fluid) energy density, $\frac{1}{2} \epsilon_{0} E_{\|}^{2}$ is the electrostatic energy density, and $S_{3, \|}$ is the total energy flux. Equation (B.18) expresses conservation of the total energy density of the system. Integrating over the periodic domain gives the total energy conservation:

$$
\partial_{t} \bar{U}_{t o t}-\bar{E}_{\|} \bar{j}_{\|}=\partial_{t} \bar{U}_{t o t}=0
$$


The $\bar{E}_{\|} \bar{j}_{\|}$term vanishes in a periodic system with no external electric field. Again, the symmetry of the moments of the temporal/spatial inertial terms is a key point. We also note that, in the discrete, the requirements for energy conservation with the inertial terms are not guaranteed to be compatible with those for momentum conservation. Further, we again observe that the second moment of the acceleration term must correspond to the current in Ampère's equation, which, as we saw in Appendix B.1 must also correspond to the zeroth moment of the advective flux. This once again presents apparently conflicting requirements for discrete conservation.

\section{Appendix C. Detailed definitions of discrete fluxes}

As we saw in section 3 the transformed Vlasov equation is discretized conservatively as

$$
\begin{aligned}
& \delta_{t} \tilde{f}_{\alpha, i, j, k}+\underbrace{\delta_{x}\left[v_{\|, \alpha, j}^{p} \overline{\left(\tilde{f}_{\alpha}^{p+1}\right)_{j, k}} v_{i}\right.}_{(a)}+\underbrace{\frac{q_{\alpha}}{m_{\alpha}} \frac{E_{\|, i}^{p+1}}{v_{\alpha, i}^{*, p}} \delta_{c_{\|}}\left[\overline{\left(\tilde{f}_{\alpha}^{p+1}\right)_{i, k}^{q_{\alpha}} E_{\|}}\right]_{j}}_{(b)}
\end{aligned}
$$

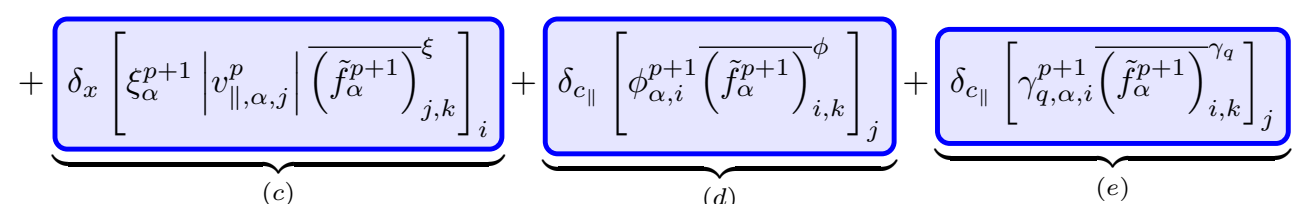

$$
\begin{aligned}
& \underbrace{-\frac{1}{v_{\alpha, i}^{*, p}} \delta_{\boldsymbol{c}} \cdot[\underbrace{\delta_{t}\left(\boldsymbol{v}_{\alpha, i}\right) \overline{\left(\tilde{f}_{\alpha}^{p+1}\right)_{i}} \delta_{t}(\boldsymbol{v})}_{\gamma_{t, \alpha, i}^{p+1}}]_{j, k}}_{(f)} \\
& \underbrace{-\frac{1}{2 v_{\alpha, i}^{*, p}} \delta_{\boldsymbol{c}} \cdot[\underbrace{}_{\gamma_{x, \alpha, i+\frac{1}{2}}^{p+1}} v_{\alpha, i+\frac{1}{2}}^{*, p} \underbrace{}_{\hat{v}_{\|, \alpha, \mathrm{eff}, i+\frac{1}{2}}^{p+1}} \delta_{x}\left[\boldsymbol{v}_{\alpha}^{p}\right]_{i+\frac{1}{2}} \overline{\left(\tilde{f}_{\alpha}^{p+1}\right)_{i}} v_{\|, \mathrm{eff}} \delta_{x}(\boldsymbol{v})]}_{(g, 1)}]_{j, k} \\
& \underbrace{-\frac{1}{2 v_{\alpha, i}^{*, p}} \delta_{\boldsymbol{c}} \cdot[\overbrace{\gamma_{x, \alpha, i-\frac{1}{2}}^{p+1}} v_{\alpha, i-\frac{1}{2}}^{*, p} \overbrace{\hat{v}_{\|, \alpha, \text { eff }, i-\frac{1}{2}}^{p+1}} \delta_{x}\left[\boldsymbol{v}_{\alpha}^{p}\right]_{i-\frac{1}{2}} \overline{\left(\tilde{f}_{\alpha}^{p+1}\right)_{i}} v_{\|, \text {eff }} \delta_{x}(\boldsymbol{v})}_{(g, 2)}]_{j, k}=0 .
\end{aligned}
$$

The various fluxes in Eq. C.1 are defined as follows. The physical configuration-space advection, $(a)$, is defined as

$$
\left[v_{\|, \alpha, j}^{p} \overline{\left(\tilde{f}_{\alpha}^{p+1}\right)_{j, k}^{v_{\|}}}\right]_{i+\frac{1}{2}}=v_{\alpha, i+\frac{1}{2}}^{*, p}\left(c_{\|, j}+\hat{u}_{\|, \alpha, i+\frac{1}{2}}^{*, p}\right) \operatorname{Interp}\left(c_{\|, j}+\hat{u}_{\|, \alpha, i+\frac{1}{2}}^{*, p}, \tilde{f}_{\alpha}^{p+1}\right)_{i+\frac{1}{2}, j, k} .
$$

Term $(b)$ - the velocity-space advection operator due to electric field acceleration - is defined as

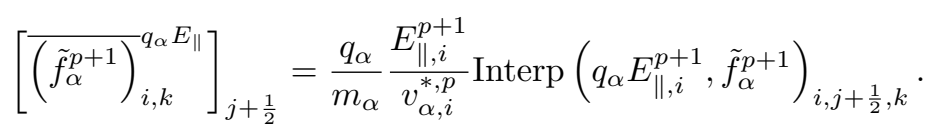

Terms $(c),(d)$, and $(e)$ are the 'pseudo-operators' introduced by the inclusion of nonlinear constraint functions, $\xi_{\alpha}$, $\phi_{\alpha}$, and $\gamma_{q, \alpha}$, which act to enforce the conservation symmetries discussed in Sec. 2.2 (see Ref. [20]). Here, we will only discuss their discrete appearance in the numerical implementation of the governing equation. The nature and definitions of these constraint functions and their respective pseudo-operators are discussed Appendix E and Sec. 4 Term $(c)$ is the pseudo-advection operator arising due to the discrete nonlinear constraint function $\xi_{\alpha}$ and is defined to be

$$
\left[\xi_{\alpha}^{p+1}\left|v_{\|, \alpha, j}^{p}\right| \overline{\left(\tilde{f}_{\alpha}^{p+1}\right)_{j, k}^{\xi}}\right]_{i+\frac{1}{2}}=v_{\alpha, i+\frac{1}{2}}^{*, p} \xi_{\alpha, i+\frac{1}{2}}^{p+1}\left|c_{\|, j}+\hat{u}_{\|, \alpha, i+\frac{1}{2}}^{*, p}\right| \operatorname{Upw}\left(\xi_{\alpha, i+\frac{1}{2}}^{p+1}, \tilde{f}_{\alpha}^{p+1}\right)_{i+\frac{1}{2}, j, k},
$$


where Upw denotes the use of straightforward upwinding based on the sign of $\xi_{\alpha, i+\frac{1}{2}}^{p+1}$. The discretization of terms $(d)$ and $(e)$ is given as

$$
\begin{gathered}
{\left[\phi_{\alpha, i}^{p+1} \overline{\left(\tilde{f}_{\alpha}^{p+1}\right)_{i, k}^{\phi}}\right]_{j+\frac{1}{2}}=\phi_{\alpha, i, j+\frac{1}{2}}^{p+1} \frac{1}{v_{\alpha, i}^{*, p}} \frac{\tilde{f}_{\alpha, i, j-\frac{1}{2}, k}^{p+1}+\tilde{f}_{\alpha, i, j+\frac{1}{2}, k}^{p+1}}{2},} \\
{\left[\gamma_{q, \alpha, i}^{p+1} \overline{\left(\tilde{f}_{\alpha}^{p+1}\right)_{i, k}^{\gamma_{q}}}\right]_{j+\frac{1}{2}}=\gamma_{q, \alpha, i, j+\frac{1}{2}}^{p+1} \frac{1}{v_{\alpha, i}^{*, p}} \frac{\tilde{f}_{\alpha, i, j-\frac{1}{2}, k}^{p+1}+\tilde{f}_{\alpha, i, j+\frac{1}{2}, k}^{p+1}}{2},}
\end{gathered}
$$

with a straighforward central differencing of $\tilde{f}_{\alpha, i, j, k}^{p+1}$ in $c_{\|}$. Recall that $\phi_{\alpha}$ and $\gamma_{q, \alpha}$ have a dependence on the parallel velocity space; the details of this dependence are given in Sec. 4

The inertial terms $(f)$ and $(g)$ arise due to the velocity-coordinate transformation. Similar to terms $(c),(d)$, and (e), they contain additional nonlinear constraint functions $\gamma_{t}$ and $\gamma_{x}$, which also act so as to enforce the continuum conservation symmetries discussed previously. The specific definitions and action of $\gamma_{t, \alpha}$ and $\gamma_{x, \alpha}$ are discussed in more detail in Sec. 4, Appendix D, and Ref. [31]. The parallel-velocity flux of the temporal inertial term, $(f)$, is defined as

$$
\begin{aligned}
-\left[\gamma_{t, \alpha, i}^{p+1} \delta_{t}\left(v_{\|, \alpha, i}^{p}\right) \overline{\left(\tilde{f}_{\alpha}^{p+1}\right)_{i}} \delta_{t}(\boldsymbol{v})\right. & \\
& = \\
& -\gamma_{t, \alpha, i, j+\frac{1}{2}, k}^{p+1} \frac{1}{v_{\alpha, i}^{*, p}} \delta_{t}\left(v_{\|, \alpha, i, j+\frac{1}{2}}^{p}\right) \operatorname{Interp}\left(-\delta_{t}\left(v_{\|, \alpha, i, j+\frac{1}{2}}^{p}\right), \tilde{f}_{\alpha}^{p+1}\right)_{i, j+\frac{1}{2}, k} .
\end{aligned}
$$

The perpendicular-velocity flux of the temporal inertial term is defined similarly:

$$
\begin{aligned}
-\left[\gamma_{t, \alpha, i}^{p+1} \delta_{t}\left(v_{\perp, \alpha, i}^{p}\right){\overline{\left(\tilde{f}_{\alpha}^{p+1}\right)_{i}}}_{\delta_{t}(\boldsymbol{v})}\right]_{j, k+\frac{1}{2}} & = \\
& -\gamma_{t, \alpha, i, j, k+\frac{1}{2}}^{p+1} \frac{1}{v_{\alpha, i}^{*, p}} \delta_{t}\left(v_{\perp, \alpha, i, k+\frac{1}{2}}^{p}\right) \operatorname{Interp}\left(-\delta_{t}\left(v_{\perp, \alpha, i, k+\frac{1}{2}}^{p}\right), \tilde{f}_{\alpha}^{p+1}\right)_{i, j, k+\frac{1}{2}}
\end{aligned}
$$

For the spatial inertial terms, $(g)$, the parallel-velocity flux is defined by

$$
\begin{aligned}
&-\left[\gamma_{x, \alpha, i+\frac{1}{2}}^{p+1} v_{\alpha, i+\frac{1}{2}}^{*, p} \hat{v}_{\|, \alpha, \mathrm{eff}, i+\frac{1}{2}}^{p+1} \delta_{x}\left[v_{\|, \alpha}^{p}\right]_{i+\frac{1}{2}}\right. \overline{\left(\tilde{f}_{\alpha}^{p+1}\right)_{i}} v_{\|, \mathrm{eff}} \delta_{x}(\boldsymbol{v}) \\
&-\gamma_{x, \alpha, i+\frac{1}{2}, j+\frac{1}{2}, k}^{p+1} v_{\alpha, i+\frac{1}{2}}^{*, p} \hat{v}_{\|, \alpha, \mathrm{eff}, i+\frac{1}{2}, j+\frac{1}{2}}^{p+1} \delta_{x}\left[v_{\|, \alpha, j+\frac{1}{2}}^{p}\right]_{i+\frac{1}{2}} \times \\
& \operatorname{Interp}\left(-\hat{v}_{\|, \alpha, \mathrm{eff}, i+\frac{1}{2}, j+\frac{1}{2}}^{p+1} \delta_{x}\left[v_{\|, \alpha, j+\frac{1}{2}}^{p}\right]_{i+\frac{1}{2}}, \tilde{f}_{\alpha}^{p+1}\right)_{i, j+\frac{1}{2}, k} .
\end{aligned}
$$

Here, we note the pseudo-flux involving the nonlinear constraint function $\xi_{\alpha, i+\frac{1}{2}}$ appears through $v_{\|, \alpha, \text { eff }, i+\frac{1}{2}, j+\frac{1}{2}}$. Similarly, the perpendicular-velocity flux is defined by

$$
\begin{aligned}
&-\left[\gamma_{x, \alpha, i+\frac{1}{2}}^{p+1} v_{\alpha, i+\frac{1}{2}}^{*, p} \hat{v}_{\|, \alpha, \mathrm{eff}, i+\frac{1}{2}}^{p+1} \delta_{x}\left[v_{\perp, \alpha}^{p}\right]_{i+\frac{1}{2}}\right. \overline{\left(\tilde{f}_{\alpha}^{p+1}\right)_{i}} v_{\|, \mathrm{eff}} \delta_{x}(\boldsymbol{v}) \\
&-\gamma_{x, \alpha, i+\frac{1}{2}, j, k+\frac{1}{2}}^{p+1} v_{\alpha, i+\frac{1}{2}}^{*, p} \hat{v}_{\|, \alpha, \mathrm{eff}, i+\frac{1}{2}, j}^{p+1} \delta_{x}\left[v_{\perp, \alpha, k+\frac{1}{2}}^{p}\right]_{i+\frac{1}{2}} \times \\
& \\
& \operatorname{Interp}\left(-\hat{v}_{\|, \alpha, \mathrm{eff}, i+\frac{1}{2}, j}^{p+1} \delta_{x}\left[v_{\perp, \alpha, k+\frac{1}{2}}^{p}\right]_{i+\frac{1}{2}}, \tilde{f}_{\alpha}^{p+1}\right)_{i, j, k+\frac{1}{2}}
\end{aligned}
$$

We note here that to evolve the normalizing speed $v_{\alpha}^{*}$ and offset velocity $u_{\|, \alpha}^{*}$ in space and time we use the same strategies as in Ref. [31]. 


\section{Appendix D. Derivation of constraint definitions for $\gamma_{t}$ and $\gamma_{x}$}

\section{Appendix D.1. Discrete momentum conservation}

First, we observe that Eqs. (B.6) and (B.7) may be discretely represented as

$$
\begin{gathered}
\left\langle v_{\|, \alpha, i, j}^{p}, \delta_{t} \tilde{f}_{\alpha, i, j, k}\right\rangle_{\delta \boldsymbol{c}}-\left\langle v_{\|, \alpha, i, j}^{p}, \frac{1}{v_{\alpha, i}^{*, p}} \delta_{\boldsymbol{c}} \cdot\left[\gamma_{t, \alpha, i}^{p+1} \delta_{t}\left(\boldsymbol{v}_{\alpha, i}\right) \overline{\left(\tilde{f}_{\alpha}^{p+1}\right)_{i}} \delta_{t}(\boldsymbol{v})\right.\right. \\
=\left\langle 1, \frac{c^{p+1} v_{\|, \alpha, i, j}^{p} \tilde{f}_{\alpha, i, j, k}^{p+1}+c^{p} v_{\|, \alpha, i, j}^{p-1} \tilde{f}_{\alpha, i, j, k}^{p}+c^{p-1} v_{\|, \alpha, i, j}^{p-2} \tilde{f}_{\alpha, i, j, k}^{p-1}}{\Delta t^{p}}\right\rangle_{\delta \boldsymbol{c}}
\end{gathered}
$$

and

$$
\begin{aligned}
& \left\langle v_{\|, \alpha, i, j}^{p}, \delta_{x}\left[v_{\alpha}^{*, p} \hat{v}_{\|, \mathrm{eff}, \alpha, j}^{p+1}{\overline{\left(\tilde{f}_{\alpha}^{p+1}\right)^{j, k}}}_{j, \mathrm{eff}}\right]_{i}\right\rangle_{\delta \boldsymbol{c}} \\
& -\left\langle v_{\|, \alpha, i, j}^{p}, \frac{1}{2 v_{\alpha, i}^{*, p}} \delta_{\boldsymbol{c}} \cdot\left[\gamma_{x, \alpha, i+\frac{1}{2}}^{p+1} v_{\alpha, i+\frac{1}{2}}^{*, p} \hat{v}_{\|, \alpha, \mathrm{eff}, i+\frac{1}{2}}^{p+1} \delta_{x}\left[\boldsymbol{v}_{\alpha}^{p}\right]_{i+\frac{1}{2}} \overline{\left(\tilde{f}_{\alpha}^{p+1}\right)_{i}^{v_{\|, \mathrm{eff}} \delta_{x}(\boldsymbol{v})}}\right]_{j, k}\right\rangle_{\delta \boldsymbol{c}} \\
& -\left\langle v_{\|, \alpha, i, j}^{p}, \frac{1}{2 v_{\alpha, i}^{*,,}} \delta_{\boldsymbol{c}} \cdot\left[\gamma_{x, \alpha, i-\frac{1}{2}}^{p+1} v_{\alpha, i-\frac{1}{2}}^{*, p} \hat{v}_{\|, \alpha, \mathrm{eff}, i-\frac{1}{2}}^{p+1} \delta_{x}\left[\boldsymbol{v}_{\alpha}^{p}\right]_{i-\frac{1}{2}} \overline{\left(\tilde{f}_{\alpha}^{p+1}\right)_{i}} v_{\|, \mathrm{eff}} \delta_{x}(\boldsymbol{v})\right]_{j, k}\right\rangle_{\delta \boldsymbol{c}} \\
& =\left\langle 1, \delta_{x}\left[\left(v_{\|, \alpha, j}^{p}\right)^{2}{\overline{\left(\tilde{f}_{\alpha}^{p+1}\right)_{j, k}}}_{v_{\|}}\right]_{i}\right\rangle_{\delta \boldsymbol{c}}+\left\langle 1, \delta_{x}\left[v_{\|, \alpha, j}^{p} \xi_{\alpha}^{p+1}\left|v_{\|, \alpha, j}^{p}\right| \overline{\left(\tilde{f}_{\alpha}^{p+1}\right)_{j, k}^{\xi}}\right]_{i}\right\rangle_{\delta \boldsymbol{c}},
\end{aligned}
$$

respectively.

If we expand the discretized form of Eq. (D.1), we find

$$
\begin{aligned}
& \left\langle v_{\|, \alpha, i, j}^{p}, \frac{c^{p+1} \tilde{f}_{\alpha, i, j, k}^{p+1}+c^{p} \tilde{f}_{\alpha, i, j, k}^{p}+c^{p-1} \tilde{f}_{\alpha, i, j, k}^{p-1}}{\Delta t^{p}}\right\rangle_{\delta \boldsymbol{c}} \\
& -\left\langle 1, \frac{c^{p+1} v_{\|, \alpha, i, j}^{p} \tilde{f}_{\alpha, i, j, k}^{p+1}+c^{p} v_{\|, \alpha, i, j}^{p-1} \tilde{f}_{\alpha, i, j, k}^{p}+c^{p-1} v_{\|, \alpha, i, j}^{p-2} \tilde{f}_{\alpha, i, j, k}^{p-1}}{\Delta t^{p}}\right\rangle_{\delta c}
\end{aligned}
$$

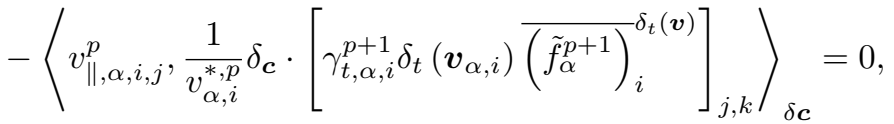

which is a concise representation of the first discrete constraint on the definition of the nonlinear constraint function $\gamma_{t}$.

To enforce Eq. D.2p, we observe that by integrating through configuration-space (i.e., sum over $\sum_{i}^{N_{x}} \Delta x$ ), the righthand side of Eq. (D.2) vanishes with periodic boundaries. Thus, expanding the individual flux terms, we find

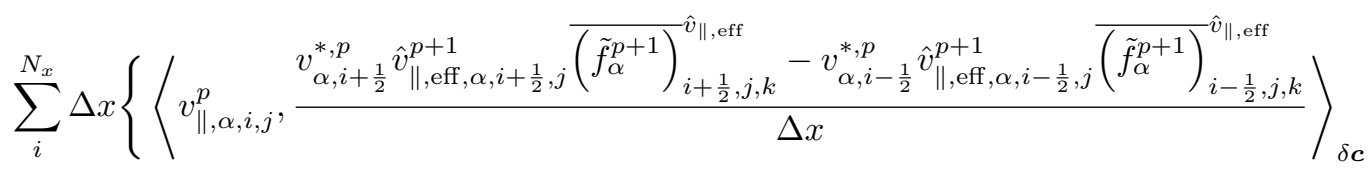

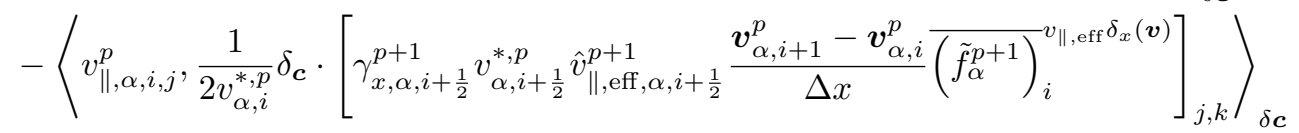

$$
\begin{aligned}
& \left.-\left\langle v_{\|, \alpha, i, j}^{p}, \frac{1}{2 v_{\alpha, i}^{*, p}} \delta_{\boldsymbol{c}} \cdot\left[\gamma_{x, \alpha, i-\frac{1}{2}}^{p+1} v_{\alpha, i-\frac{1}{2}}^{*, p} \hat{v}_{\|, \mathrm{eff}, \alpha, i-\frac{1}{2}}^{p+1} \frac{\boldsymbol{v}_{\alpha, i}^{p}-\boldsymbol{v}_{\alpha, i-1}^{p}}{\Delta x}{\overline{\left(\tilde{f}_{\alpha}^{p+1}\right)_{i}}}_{i, \mathrm{eff} \delta_{x}(\boldsymbol{v})}\right]_{j, k}\right\rangle_{\delta \boldsymbol{c}}\right\}=0 .
\end{aligned}
$$


Recall we have defined the effective velocity

$$
\hat{v}_{\|, \mathrm{eff}, i+\frac{1}{2}, j}^{p+1} \equiv\left(c_{\|, j}+\hat{u}_{\|, \alpha, i+\frac{1}{2}}^{*, p}+\xi_{\alpha, i+\frac{1}{2}}^{p+1}\left|c_{\|, j}+\hat{u}_{\|, \alpha, i+\frac{1}{2}}^{*, p}\right|\right) .
$$

Note that in the case of $\hat{v}_{\|, \text {eff }, \alpha, i+\frac{1}{2}, j}^{p+1} \overline{\left(\tilde{f}_{\alpha}^{p+1}\right)_{\|+\frac{1}{2}, j, k}} \hat{v}_{\|, \text {eff }}$ in the first term of Eq. D.4p, this is simply a shorthand for the summation of the individually interpolated fluxes $\left[v_{\|, \alpha, j}^{p} \overline{\left(\tilde{f}_{\alpha}^{p+1}\right)_{j, k}}\right]_{i+\frac{1}{2}}$ and $\left[\xi_{\alpha}^{p+1}\left|v_{\|, \alpha, j}^{p}\right| \overline{\left(\tilde{f}_{\alpha}^{p+1}\right)_{j, k}^{\xi}}\right]_{i+\frac{1}{2}}$. If we then telescope the summation in Eq. (D.4) in configuration-space, we find

$$
\begin{aligned}
& \sum_{i}^{N_{x}}\left\{\left\langle v_{\|, \alpha, i, j}^{p}-v_{\|, \alpha, i+1, j}^{p}, \frac{1}{\Delta x} v_{\alpha, i+\frac{1}{2}}^{*, p} \hat{v}_{\|, \text {eff }, \alpha, i+\frac{1}{2}, j}^{p+1} \overline{\left(\tilde{f}_{\alpha}^{p+1}\right)_{i+\frac{1}{2}, j, k}^{\hat{v}_{\|, \mathrm{eff}}}}\right\rangle_{\delta \boldsymbol{c}}\right. \\
& -\left\langle v_{\|, \alpha, i, j}^{p}, \frac{1}{2 v_{\alpha, i}^{*, p}} \delta_{\boldsymbol{c}} \cdot\left[\gamma_{x, \alpha, i+\frac{1}{2}}^{p+1} v_{\alpha, i+\frac{1}{2}}^{*, p} \hat{v}_{\|, \mathrm{eff}, i+\frac{1}{2}} \delta_{x}\left(\boldsymbol{v}^{p}\right)_{i+\frac{1}{2}} \overline{\left(\tilde{f}_{\alpha}^{p+1}\right)_{i}^{v_{\|, \mathrm{eff}} \delta_{x}(\boldsymbol{v})}}\right]_{j, k}\right\rangle_{\delta \boldsymbol{c}} \\
& \left.-\left\langle v_{\|, \alpha, i+1, j}^{p}, \frac{1}{2 v_{\alpha, i+1}^{*, p}} \delta_{\boldsymbol{c}} \cdot\left[\gamma_{x, \alpha, i+\frac{1}{2}}^{p+1} v_{\alpha, i+\frac{1}{2}}^{*, p} \hat{v}_{\|, \mathrm{eff}, i+\frac{1}{2}} \delta_{x}\left(\boldsymbol{v}^{p}\right)_{i+\frac{1}{2}} \overline{\left(\tilde{f}_{\alpha}^{p+1}\right)_{i+1}^{v_{\|, \mathrm{eff}} \delta_{x}(\boldsymbol{v})}}\right]_{j, k}\right\rangle_{\delta \boldsymbol{c}}\right\}=0,
\end{aligned}
$$

where the discrete constraint on $\gamma_{x, \alpha}$ is found by enforcing that

$$
\begin{aligned}
& \left\langle v_{\|, \alpha, i, j}^{p}-v_{\|, \alpha, i+1, j}^{p}, \frac{1}{\Delta x} v_{\alpha, i+\frac{1}{2}}^{*, p} \hat{v}_{\|, \text {eff }, \alpha, i+\frac{1}{2}, j}^{p+1} \overline{\left(\tilde{f}_{\alpha}^{p+1}\right)_{i+\frac{1}{2}, j, k}^{\hat{v}_{\|, \mathrm{eff}}}}\right\rangle_{\delta \boldsymbol{c}} \\
& -\left\langle v_{\|, \alpha, i, j}^{p}, \frac{1}{2 v_{\alpha, i}^{*, p}} \delta_{\boldsymbol{c}} \cdot\left[\gamma_{x, \alpha, i+\frac{1}{2}}^{p+1} v_{\alpha, i+\frac{1}{2}}^{*, p} \hat{v}_{\|, \mathrm{eff}, i+\frac{1}{2}} \delta_{x}\left(\boldsymbol{v}^{p}\right)_{i+\frac{1}{2}}{\overline{\left(\tilde{f}_{\alpha}^{p+1}\right)_{i}}}_{v_{\|, \mathrm{eff}} \delta_{x}(\boldsymbol{v})}\right]_{j, k}\right\rangle_{\delta \boldsymbol{c}} \\
& -\left\langle v_{\|, \alpha, i+1, j}^{p}, \frac{1}{2 v_{\alpha, i+1}^{*, p}} \delta_{\boldsymbol{c}} \cdot\left[\gamma_{x, \alpha, i+\frac{1}{2}}^{p+1} v_{\alpha, i+\frac{1}{2}}^{*, p} \hat{v}_{\|, \mathrm{eff}, i+\frac{1}{2}} \delta_{x}\left(\boldsymbol{v}^{p}\right)_{i+\frac{1}{2}}{\overline{\left(\tilde{f}_{\alpha}^{p+1}\right)}}_{i+1}^{v_{\|, \mathrm{eff}} \delta_{x}(\boldsymbol{v})}\right]_{j, k}\right\rangle_{\delta \boldsymbol{c}}=0
\end{aligned}
$$

is zero for each cell-face $i+\frac{1}{2}$.

Appendix D.2. Discrete energy conservation

We first observe that Eqs. B.13 and B.14 may be discretely represented as

$$
\begin{gathered}
\left\langle m_{\alpha} \frac{1}{2}\left(\boldsymbol{v}_{\alpha, i, j, k}^{p}\right)^{2}, \delta_{t} \tilde{f}_{\alpha, i, j, k}\right\rangle_{\delta \boldsymbol{c}}-\left\langle m_{\alpha} \frac{1}{2}\left(\boldsymbol{v}_{\alpha, i, j, k}^{p}\right)^{2}, \frac{1}{v_{\alpha, i}^{*, p}} \delta_{\boldsymbol{c}} \cdot\left[\gamma_{t, \alpha, i}^{p+1} \delta_{t}\left(\boldsymbol{v}_{\alpha, i}\right) \overline{\left(\tilde{f}_{\alpha}^{p+1}\right)_{i}^{\delta_{t}(\boldsymbol{v})}}\right]_{j, k}\right\rangle_{\delta \boldsymbol{c}} \\
=\left\langle 1, m_{\alpha} \frac{1}{2} \frac{c^{p+1}\left(\boldsymbol{v}_{\alpha, i, j, k}^{p}\right)^{2} \tilde{f}_{\alpha, i, j, k}^{p+1}+c^{p}\left(\boldsymbol{v}_{\alpha, i, j, k}^{p-1}\right)^{2} \tilde{f}_{\alpha, i, j, k}^{p}+c^{p-1}\left(\boldsymbol{v}_{\alpha, i, j, k}^{p-2}\right)^{2} \tilde{f}_{\alpha, i, j, k}^{p-1}}{\Delta t^{p}}\right\rangle_{\delta \boldsymbol{c}},
\end{gathered}
$$


and

$$
\begin{aligned}
& \left\langle m_{\alpha} \frac{1}{2}\left(\boldsymbol{v}_{\alpha, i, j, k}^{p}\right)^{2}, \delta_{x}\left[v_{\|, \alpha, j}^{p}{\overline{\left(\tilde{f}_{\alpha}^{p+1}\right)_{j, k}}}_{v_{\|}}\right]_{i}\right\rangle_{\delta \boldsymbol{c}}+\left\langle m_{\alpha} \frac{1}{2}\left(\boldsymbol{v}_{\alpha, i, j, k}^{p}\right)^{2}, \delta_{x}\left[\xi_{\alpha}^{p+1}\left|v_{\|, \alpha, j}^{p}\right| \overline{\left(\tilde{f}_{\alpha}^{p+1}\right)_{j, k}^{\xi}}\right]_{i}\right\rangle_{\delta \boldsymbol{c}} \\
& +\left\langle m_{\alpha} \frac{1}{2}\left(\boldsymbol{v}_{\alpha, i, j, k}^{p}\right)^{2}, \frac{J_{x, \|, \alpha, i, j+\frac{1}{2}, k}^{p+1}-J_{x, \|, \alpha, i, j-\frac{1}{2}, k}^{p+1}}{\Delta c_{\|}}\right\rangle_{\delta \boldsymbol{c}}
\end{aligned}
$$

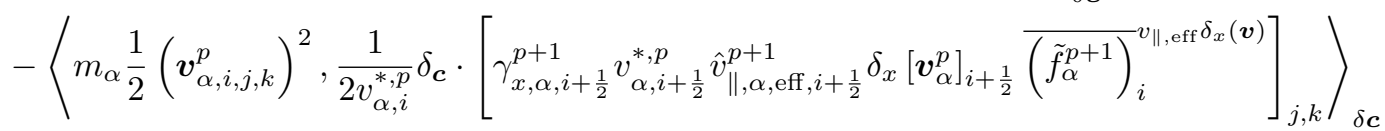

$$
\begin{aligned}
& -\left\langle m_{\alpha} \frac{1}{2}\left(\boldsymbol{v}_{\alpha, i, j, k}^{p}\right)^{2}, \frac{1}{2 v_{\alpha, i}^{*, p}} \delta_{\boldsymbol{c}} \cdot\left[\gamma_{x, \alpha, i-\frac{1}{2}}^{p+1} v_{\alpha, i-\frac{1}{2}}^{*, p} \hat{v}_{\|, \alpha, \mathrm{eff}, i-\frac{1}{2}}^{p+1} \delta_{x}\left[\boldsymbol{v}_{\alpha}^{p}\right]_{i-\frac{1}{2}} \overline{\left(\tilde{f}_{\alpha}^{p+1}\right)_{i}} v_{\|, \mathrm{eff}} \delta_{x}(\boldsymbol{v})\right]_{j, k}\right\rangle_{\delta \boldsymbol{c}} \\
& =\left\langle 1, \delta_{x}\left[m_{\alpha} \frac{1}{2}\left(\boldsymbol{v}_{\alpha, j, k}^{p}\right)^{2} v_{\|, \alpha, j}^{p}{\overline{\left(\tilde{f}_{\alpha}^{p+1}\right)_{j, k}}}_{v_{\|}}\right]_{\delta \boldsymbol{c}}\right. \\
& +\left\langle 1, \delta_{x}\left[m_{\alpha} \frac{1}{2}\left(\boldsymbol{v}_{\alpha, j, k}^{p}\right)^{2} \xi_{\alpha}^{p+1}\left|v_{\|, \alpha, j}^{p}\right| \overline{\left(\tilde{f}_{\alpha}^{p+1}\right)_{j, k}^{\xi}}\right]_{i}\right\rangle_{\delta \boldsymbol{c}} .
\end{aligned}
$$

If we expand Eq. (D.7), we find

$$
\begin{aligned}
& \left\langle m_{\alpha} \frac{1}{2}\left(\boldsymbol{v}_{\alpha, i, j, k}^{p}\right)^{2}, \frac{c^{p+1} \tilde{f}_{\alpha, i, j, k}^{p+1}+c^{p} \tilde{f}_{\alpha, i, j, k}^{p}+c^{p-1} \tilde{f}_{\alpha, i, j, k}^{p-1}}{\Delta t^{p}}\right\rangle_{\delta \boldsymbol{c}} \\
& -\left\langle 1, \frac{c^{p+1} m_{\alpha} \frac{1}{2}\left(\boldsymbol{v}_{\alpha, i, j, k}^{p}\right)^{2} \tilde{f}_{\alpha, i, j, k}^{p+1}+c^{p} m_{\alpha} \frac{1}{2}\left(\boldsymbol{v}_{\alpha, i, j, k}^{p-1}\right)^{2} \tilde{f}_{\alpha, i, j, k}^{p}+c^{p-1} m_{\alpha} \frac{1}{2}\left(\boldsymbol{v}_{\alpha, i, j, k}^{p-2}\right)^{2} \tilde{f}_{\alpha, i, j, k}^{p-1}}{\Delta t^{p}}\right\rangle_{\delta \boldsymbol{c}} \\
& -\left\langle m_{\alpha} \frac{1}{2}\left(\boldsymbol{v}_{\alpha, i, j, k}^{p}\right)^{2}, \frac{1}{v_{\alpha, i}^{*, p}} \delta_{\boldsymbol{c}} \cdot\left[\gamma_{t, \alpha, i}^{p+1} \delta_{t}\left(\boldsymbol{v}_{\alpha, i}\right) \overline{\left(\tilde{f}_{\alpha}^{p+1}\right)_{i}} \delta_{t}(\boldsymbol{v})\right]_{j, k}\right\rangle_{\delta \boldsymbol{c}}=0
\end{aligned}
$$

which is a concise representation of the final discrete constraint on the definition of the nonlinear constraint function $\gamma_{t}$.

As in the case of momentum conservation, Eq. (D.8) must be enforced more carefully. Once again we will integrate through configuration-space and assume periodic boundaries, whereupon we arrive at

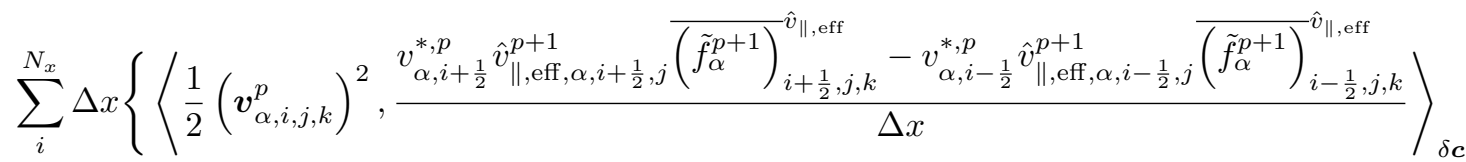

$$
\begin{aligned}
& -\left\langle\frac{1}{2}\left(\boldsymbol{v}_{\alpha, i, j, k}^{p}\right)^{2}, \frac{1}{2 v_{\alpha, i}^{*, p}} \delta_{\boldsymbol{c}} \cdot\left[\gamma_{x, \alpha, i+\frac{1}{2}}^{p+1} v_{\alpha, i+\frac{1}{2}}^{*, p} \hat{v}_{\|, \text {eff }, \alpha, i+\frac{1}{2}}^{p+1} \frac{\boldsymbol{v}_{\alpha, i+1}^{p}-\boldsymbol{v}_{\alpha, i}^{p}}{\Delta x} \overline{\left(\tilde{f}_{\alpha}^{p+1}\right)_{i}} v_{\|, \mathrm{eff}} \delta_{x}(\boldsymbol{v})\right]_{j, k}\right\rangle_{\delta \boldsymbol{c}}
\end{aligned}
$$

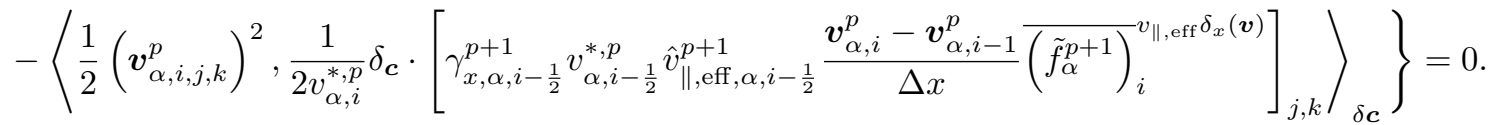


Again telescoping the summation in configuration-space and equating the quantity inside braces to zero, we find

$$
\begin{aligned}
& \left\langle\frac{\left(\boldsymbol{v}_{\alpha, i, j, k}^{p}\right)^{2}}{2}-\frac{\left(\boldsymbol{v}_{\alpha, i+1, j, k}^{p}\right)^{2}}{2}, \frac{1}{\Delta x} v_{\alpha, i+\frac{1}{2}}^{*, p} \hat{v}_{\|, \mathrm{eff}, \alpha, i+\frac{1}{2}, j}^{p+1} \overline{\left(\tilde{f}_{\alpha}^{p+1}\right)_{i+\frac{1}{2}, j, k}} \hat{v}_{\|, \mathrm{eff}}\right\rangle \\
& \quad-\left\langle\frac{\left(\boldsymbol{v}_{\alpha, i, j, k}^{p}\right)^{2}}{2}, \frac{1}{2 v_{\alpha, i}^{*, p}} \delta_{\boldsymbol{c}} \cdot\left[\gamma_{x, \alpha, i+\frac{1}{2}}^{p+1} v_{\alpha, i+\frac{1}{2}}^{*, p} \hat{v}_{\|, \mathrm{eff}, i+\frac{1}{2}} \delta_{x}\left(\boldsymbol{v}^{p}\right)_{i+\frac{1}{2}} \overline{\left(\tilde{f}_{\alpha}^{p+1}\right)_{i}} v_{\|, \mathrm{eff}} \delta_{x}(\boldsymbol{v})\right]_{j, k}\right\rangle_{\delta \boldsymbol{c}} \\
& \quad-\left\langle\frac{\left(\boldsymbol{v}_{\alpha, i+1, j, k}^{p}\right)^{2}}{2} \frac{1}{2 v_{\alpha, i+1}^{*, p}} \delta_{\boldsymbol{c}} \cdot\left[\gamma_{x, \alpha, i+\frac{1}{2}}^{p+1} v_{\alpha, i+\frac{1}{2}}^{*, p} \hat{v}_{\|, \mathrm{eff}, i+\frac{1}{2}} \delta_{x}\left(\boldsymbol{v}^{p}\right)_{i+\frac{1}{2}} \frac{\left(\tilde{f}_{\alpha}^{p+1}\right)_{i+1}}{\left.v_{\|, \mathrm{eff}} \delta_{x}(\boldsymbol{v})\right]_{j, k}}\right\rangle_{\delta \boldsymbol{c}}=0\right.
\end{aligned}
$$

which is the final discrete constraint on $\gamma_{x}$.

\section{Appendix E. Derivation of constraint definitions for $\xi, \phi$, and $\gamma_{q}$}

\section{Appendix E.1. Discrete charge \& mass conservation}

To demonstrate a discrete mass conservation, we apply to Eq. $\sqrt{23}$ the discrete moment $\left\langle m_{\alpha}, \cdots\right\rangle_{\delta \boldsymbol{c}}$. First, we observe that with appropriate discrete boundary conditions (i.e., zero mass flux in the velocity space and periodic boundaries in the configuration space), all velocity-space divergence terms vanish under the discrete moment (as in the continuum case). Thus, we are left with

$$
\left\langle m_{\alpha}, \delta_{t} \tilde{f}_{\alpha, i, j, k}\right\rangle_{\delta \boldsymbol{c}}+\left\langle m_{\alpha}, \delta_{x}\left[v_{\|, \alpha, j}^{p}{\overline{\left(\tilde{f}_{\alpha}^{p+1}\right)_{j, k}}}_{v_{i}}\right]_{\delta \boldsymbol{c}}+\left\langle m_{\alpha}, \delta_{x}\left[\xi_{\alpha}^{p+1}\left|v_{\|, \alpha, j}^{p}\right| \overline{\left(\tilde{f}_{\alpha}^{p+1}\right)_{j, k}^{\xi}}\right]_{i}\right\rangle_{\delta \boldsymbol{c}}=0\right.
$$

Clearly, if we sum over all species $\alpha$ and integrate over the configuration space, $\sum_{\alpha}^{N_{s p}} \sum_{i=1}^{N_{x}}$, we will obtain the proper discrete mass conservation (assuming a periodic domain in the configuration space):

$$
\delta_{t} M_{t o t}=\frac{c^{p+1} M_{t o t}^{p+1}+c^{p} M_{t o t}^{p}+c^{p-1} M_{t o t}^{p-1}}{\Delta t^{p}}=0
$$

where $M_{t o t}^{p} \equiv \sum_{\alpha}^{N_{s p}} \sum_{i=1}^{N_{x}} m_{\alpha} n_{\alpha, i}^{p}=\sum_{\alpha}^{N_{s p}} \sum_{i=1}^{N_{x}} m_{\alpha}\left\langle\tilde{f}_{\alpha, i, j, k}^{p}\right\rangle_{\boldsymbol{v}_{j, k}}$. Defining the discrete moments

$$
\begin{aligned}
n_{\alpha, i}^{p+1} & \equiv\left\langle 1, \tilde{f}_{\alpha, i, j, k}^{p+1}\right\rangle_{\delta \boldsymbol{c}}, \\
\widetilde{\Gamma}_{\|, \alpha, i+\frac{1}{2}}^{p+1} & \equiv\left\langle 1, v_{\alpha, i+\frac{1}{2}}^{*, p}\left(c_{\|, j}+\hat{u}_{\|, \alpha, i+\frac{1}{2}}^{*, p}\right) \overline{\left.\left(\tilde{f}_{\alpha}^{p+1}\right)_{i+\frac{1}{2}, j, k}^{v_{\|, i+\frac{1}{2}, j}}\right\rangle_{\delta \boldsymbol{c}},}\right. \\
\Pi_{\xi, \|, \alpha, i+\frac{1}{2}}^{p+1} & \equiv\left\langle 1, v_{\alpha, i+\frac{1}{2}}^{*, p}\left|c_{\|, j}+\hat{u}_{\|, \alpha, i+\frac{1}{2}}^{*, p}\right| \overline{\left.\left(\tilde{f}_{\alpha}^{p+1}\right)_{i+\frac{1}{2}, j, k}^{\xi_{\alpha, i+\frac{1}{2}}^{p+1}}\right\rangle_{\delta \boldsymbol{c}},}\right.
\end{aligned}
$$

we may further express Eq. [E.1] in terms of discrete moment quantities:

$$
\begin{aligned}
& m_{\alpha} \frac{c^{p+1} n_{\alpha, i}^{p+1}+c^{p} n_{\alpha, i}^{p}+c^{p-1} n_{\alpha, i}^{p-1}}{\Delta t^{p}} \\
&+m_{\alpha}\left(\frac{\widetilde{\Gamma}_{\|, \alpha, i+\frac{1}{2}}^{p+1}-\widetilde{\Gamma}_{\|, \alpha, i-\frac{1}{2}}^{p+1}}{\Delta x}+\frac{\xi_{\alpha, i+\frac{1}{2}}^{p+1} \Pi_{\xi, \|, \alpha, i+\frac{1}{2}}^{p+1}-\xi_{\alpha, i-\frac{1}{2}}^{p+1} \Pi_{\xi, \|, \alpha, i-\frac{1}{2}}^{p+1}}{\Delta x}\right)=0 .
\end{aligned}
$$


If we recall Sec. Appendix B.1, we know that the species particle flux density density that forms the current density in Ampère's equation must be identical to the momentum density that appears in the continuity equation. We observe that the discrete particle flux density forming the current in Ampère's equation, $\widehat{\Gamma}_{\|, \alpha, i+\frac{1}{2}}^{p+1}$, must therefore be

$$
\widehat{\Gamma}_{\|, \alpha, i+\frac{1}{2}}^{p+1}=\widetilde{\Gamma}_{\|, \alpha, i+\frac{1}{2}}^{p+1}+\xi_{\alpha, i+\frac{1}{2}}^{p+1} \Pi_{\xi, \|, \alpha, i+\frac{1}{2}}^{p+1} .
$$

Thus, the purpose of the nonlinear constraint function $\xi$ is to enforce that the truncation error between the discrete representations of particle flux density, $\widetilde{\Gamma}_{\|, \alpha, i+\frac{1}{2}}$ and $\widehat{\Gamma}_{\|, \alpha, i+\frac{1}{2}}$, vanishes. The precise discrete definition of $\widehat{\Gamma}_{\|, \alpha, i+\frac{1}{2}}$ is given in Appendix E.3. The constraint function $\xi$ and its 'pseudo-advection' operator are critical to enforcing charge conservation, as we will see in Sec. 6.3 .

\section{Appendix E.2. Discrete momentum conservation}

To demonstrate a discrete momentum conservation, we apply to Eq. 23 the discrete moment $\left\langle m_{\alpha} v_{\|, \alpha, i, j}^{p}, \cdots\right\rangle_{\delta c}$, and note that $\boldsymbol{v}_{\alpha, i, j, k}^{p}=v_{\alpha, i}^{*, p}\left(\boldsymbol{c}_{j, k}+\boldsymbol{e}_{\|} \hat{u}_{\|, \alpha, i}^{*, p}\right)$, i.e., $v_{\|, \alpha, i, j}^{p}=v_{\alpha, i}^{*, p}\left(c_{\|, j}+\hat{u}_{\|, \alpha, i}^{*, p}\right)$ and $v_{\perp, \alpha, i, k}^{p}=v_{\alpha, i}^{*, p} \tilde{v}_{\perp, k}$. Employing the discrete moment in the configuration space, we find

$$
\begin{gathered}
m_{\alpha} \sum_{i}^{N_{x}} \Delta x\left\{\frac{c^{p+1} \Gamma_{\|, \alpha, i}^{p+1}+c^{p} \Gamma_{\|, \alpha, i}^{p}+c^{p-1} \Gamma_{\|, \alpha, i}^{p-1}}{\Delta t^{p}}+\left\langle v_{\|, \alpha, i, j}^{p}, \frac{q_{\alpha}}{m_{\alpha}} \frac{E_{\|, i}^{p+1}}{v_{\alpha, i}^{*, p}} \delta_{c_{\|}}\left[\overline{\left.\left(\tilde{f}_{\alpha}^{p+1}\right)_{i, k}^{q_{\alpha} E_{\|}}\right]_{j}}\right\rangle_{\delta \boldsymbol{c}}\right.\right. \\
\left.+\left\langle v_{\|, \alpha, i, j}^{p}, \delta_{c_{\|}}\left[\phi_{\alpha, i}^{p+1} \overline{\left(\tilde{f}_{\alpha}^{p+1}\right)_{i, k}^{\phi}}\right]_{j}\right\rangle_{\delta \boldsymbol{c}}+\left\langle v_{\|, \alpha, i, j}^{p}, \delta_{c_{\|}}\left[\gamma_{q, \alpha, i}^{p+1} \overline{\left(\tilde{f}_{\alpha}^{p+1}\right)_{i, k}^{\gamma_{q}}}\right]_{j}\right\rangle_{\delta \boldsymbol{c}}\right\}=0,
\end{gathered}
$$

where we have defined

$$
\Gamma_{\|, \alpha, i}^{p+1} \equiv\left\langle 1, v_{\|, \alpha, i, j}^{p} \tilde{f}_{\alpha, i, j, k}^{p+1}\right\rangle_{\delta \boldsymbol{c}} .
$$

Expanding the individual flux operators and defining the discrete number density based on the moment of the acceleration operator,

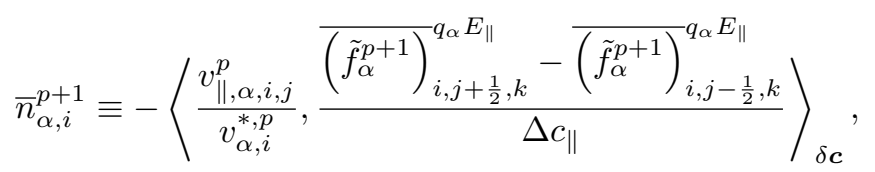

we find

$$
\begin{aligned}
m_{\alpha} \sum_{i}^{N_{x}} \Delta x\left\{\frac{c^{p+1} \Gamma_{\|, \alpha, i}^{p+1}+c^{p} \Gamma_{\|, \alpha, i}^{p}+c^{p-1} \Gamma_{\|, \alpha, i}^{p-1}}{\Delta t^{p}}\right. & -\bar{n}_{\alpha, i}^{p+1} E_{\|, i}^{p+1} \frac{q_{\alpha}}{m_{\alpha}} \\
& \left.-\left(\phi_{\alpha, i}^{+, p+1}+1\right) n_{\alpha, i}^{+, p+1}-\left(\gamma_{q, \alpha, i}^{-, p+1}+1\right) n_{\alpha, i}^{-, p+1}\right\}=0,
\end{aligned}
$$

Here, we defined $\phi$ and $\gamma_{q}$ to be split in $v_{\|}$-space as

$$
\begin{aligned}
\phi_{\alpha, i, j+\frac{1}{2}}^{p+1} & = \begin{cases}\phi_{\alpha, i}^{+, p+1} & \text { if } v_{\|, \alpha, i, j+\frac{1}{2}} \geq u_{\|, \alpha, i}^{p}, \\
1 & \text { otherwise }\end{cases} \\
\gamma_{q, \alpha, i, j+\frac{1}{2}}^{p+1} & = \begin{cases}1 & \text { if } v_{\|, \alpha, i, j+\frac{1}{2}} \geq u_{\|, \alpha, i}^{p}, \\
\gamma_{q, \alpha, i}^{-, p+1} & \text { otherwise }\end{cases}
\end{aligned}
$$

where the definitions for $\phi_{\alpha, i}^{+, p+1}$ and $\gamma_{q, \alpha, i}^{-, p+1}$ will be determined shortly. The rationale for splitting $\phi$ and $\gamma_{q}$ in this way is, as shall be seen in Sec. Appendix E.3. for solvability of the resulting $2 \times 2$ linear system from which $\phi$ and $\gamma_{q}$ 
are calculated. From this splitting, we define the "upper" and "lower" densities as

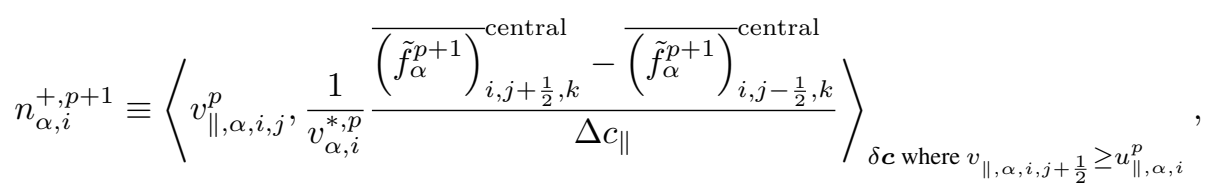

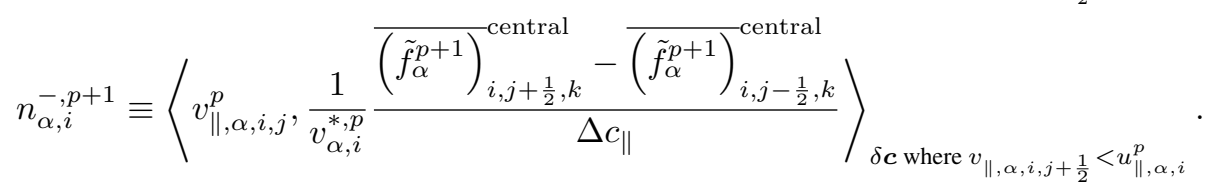

If we then sum Eq. (E.9) over all species $\alpha$, we find

$$
\delta_{t} P_{\text {total }}-\sum_{\alpha}^{N_{s p}} m_{\alpha} \sum_{i}^{N_{x}} \Delta x\left\{\bar{n}_{\alpha, i}^{p+1} E_{\|, i}^{p+1} \frac{q_{\alpha}}{m_{\alpha}}+\left(\phi_{\alpha, i}^{+, p+1}+1\right) n_{\alpha, i}^{+, p+1}+\left(\gamma_{q, \alpha, i}^{-, p+1}+1\right) n_{\alpha, i}^{-, p+1}\right\}=0
$$

where

$$
\delta_{t} P_{\text {total }} \equiv \sum_{\alpha}^{N_{s p}} m_{\alpha} \sum_{i}^{N_{x}} \Delta x \frac{c^{p+1} \Gamma_{\|, \alpha, i}^{p+1}+c^{p} \Gamma_{\|, \alpha, i}^{p}+c^{p-1} \Gamma_{\|, \alpha, i}^{p-1}}{\Delta t^{p}}
$$

is the discrete time derivative of the total momentum of the system. Recalling the symmetry with Gauss' law in Eq. B.10, we realize that Eq. E.13, must become

$$
\delta_{t} P_{\text {total }}-\sum_{\alpha}^{N_{s p}} m_{\alpha} \sum_{i}^{N_{x}} \Delta x\left\{n_{\alpha, i}^{p+1} E_{\|, i}^{p+1} \frac{q_{\alpha}}{m_{\alpha}}\right\}=\delta_{t} P_{\text {total }}-\sum_{i}^{N_{x}} \Delta x\left\{\epsilon_{0} E_{\|, i}^{p+1} \frac{E_{\|, i+\frac{1}{2}}^{p+1}-E_{\|, i-\frac{1}{2}}^{p+1}}{\Delta x}\right\}=0,
$$

where the final summation vanishes - assuming periodic boundaries - when we recall that $E_{\|, i}^{p+1}$ is the average of adjacent cell-face values. Thus we achieve $\delta_{t} P_{\text {total }}=0$, which is a discrete statement of momentum conservation. The equivalence between Eqs. E.13) and (E.14) provides us the first discrete constraint for the nonlinear constraint functions $\phi_{\alpha}$ and $\gamma_{q, \alpha}$ :

$$
\left(\phi_{\alpha, i}^{+, p+1}+1\right) n_{\alpha, i}^{+, p+1}+\left(\gamma_{q, \alpha, i}^{-, p+1}+1\right) n_{\alpha, i}^{-, p+1}=\left(n_{\alpha, i}^{p+1}-\bar{n}_{\alpha, i}^{p+1}\right) E_{\|, i}^{p+1} \frac{q_{\alpha}}{m_{\alpha}} .
$$

Thus, $\phi$ and $\gamma_{q}$ act in concert to enforce that the truncation error between the two discrete representations of density, $n_{\alpha, i}$ and $\bar{n}_{\alpha, i}$, vanishes.

\section{Appendix E.3. Discrete energy conservation}

To demonstrate discrete energy conservation, we apply the discrete moment $\left\langle m_{\alpha} \frac{1}{2}\left(\boldsymbol{v}_{\alpha, i, j, k}^{p}\right)^{2}, \cdots\right\rangle_{\delta \boldsymbol{c}}$ to Eq. 23, where $\frac{1}{2}\left(\boldsymbol{v}_{\alpha, i, j, k}^{p}\right)^{2}=\frac{1}{2}\left[\left(v_{\|, \alpha, i, j}^{p}\right)^{2}+\left(v_{\perp, \alpha, i, k}^{p}\right)^{2}\right]$. Utilizing the preceding developments and evaluating the discrete moment in the configuration space while summing over all species, we find

$$
\begin{aligned}
& \delta_{t} U_{\text {fluid }}+\sum_{\alpha} \sum_{i} \sum_{s p} \Delta x\left\{\left\langlem_{\alpha} \frac{1}{2}\left(\boldsymbol{v}_{\alpha, i, j, k}^{p}\right)^{2},\right.\right.\left.\frac{q_{\alpha}}{m_{\alpha}} \frac{E_{\|, i}^{p+1}}{v_{\alpha, i}^{*, p}} \delta_{c_{\|}}\left[\overline{\left(\tilde{f}_{\alpha}^{p+1}\right)_{i, k}^{q_{\alpha}} E_{\|}}\right]_{j}\right\rangle_{\delta \boldsymbol{c}} \\
&+\left\langle m_{\alpha} \frac{1}{2}\left(\boldsymbol{v}_{\alpha, i, j, k}^{p}\right)^{2}, \delta_{c_{\|}}\left[\phi_{\alpha, i}^{p+1} \overline{\left(\tilde{f}_{\alpha}^{p+1}\right)_{i, k}^{\phi}}\right]_{j}\right\rangle_{\delta \boldsymbol{c}} \\
&\left.+\left\langle m_{\alpha} \frac{1}{2}\left(\boldsymbol{v}_{\alpha, i, j, k}^{p}\right)^{2}, \delta_{c_{\|}}\left[\gamma_{q, \alpha, i}^{p+1} \overline{\left(\tilde{f}_{\alpha}^{p+1}\right)_{i, k}^{\gamma_{q}}}\right]_{j}\right\rangle_{\delta \boldsymbol{c}}\right\}=0
\end{aligned}
$$


where we defined the quantities

$$
\delta_{t} U_{\text {fluid }} \equiv \sum_{\alpha}^{N_{s p}} \sum_{i}^{N_{x}} \Delta x \frac{c^{p+1} \varepsilon_{\alpha, i}^{p+1}+c^{p} \varepsilon_{\alpha, i}^{p}+c^{p-1} \varepsilon_{\alpha, i}^{p-1}}{\Delta t^{p}},
$$

and

$$
\varepsilon_{\alpha, i}^{p+1} \equiv\left\langle 1, m_{\alpha} \frac{1}{2}\left(\boldsymbol{v}_{\alpha, i, j, k}^{p}\right)^{2} \tilde{f}_{\alpha, i, j, k}^{p+1}\right\rangle_{\boldsymbol{v}_{j, k}} .
$$

Again, expanding the individual flux operators and defining the discrete particle flux density obtained from the moment of the acceleration operator,

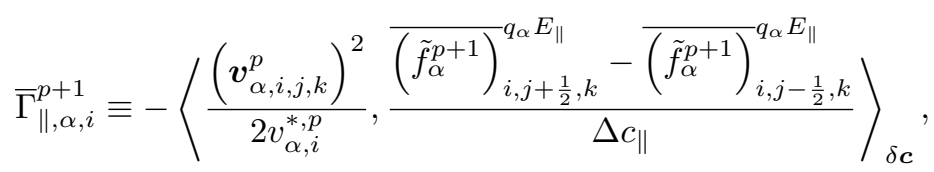

we obtain

$$
\begin{aligned}
\delta_{t} U_{\text {fluid }}-\sum_{\alpha}^{N_{s p}} \sum_{i}^{N_{x}} \Delta x\left\{q_{\alpha} E_{\|, i}^{p+1} \bar{\Gamma}_{\|, \alpha, i}^{p+1}\right. & \\
& \left.+m_{\alpha}\left[\left(\phi_{\alpha, i}^{+, p+1}+1\right) \Gamma_{\|, \alpha, i}^{+, p+1}+\left(\gamma_{q, \alpha, i}^{-, p+1}+1\right) \Gamma_{\|, \alpha, i}^{-, p+1}\right]\right\}=0 .
\end{aligned}
$$

Here, as before, we defined the "upper" and "lower" momenta as

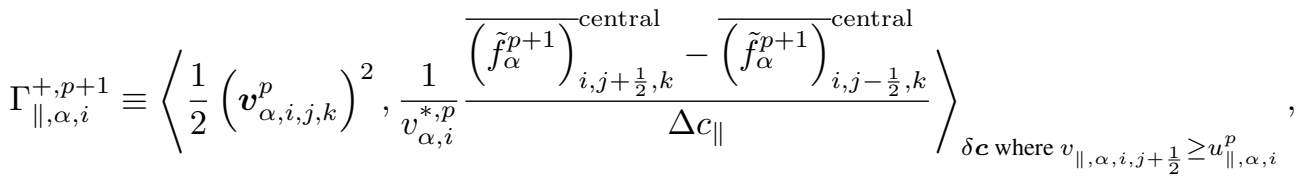

$$
\begin{aligned}
& \Gamma_{\|, \alpha, i}^{-, p+1} \equiv\left\langle\frac{1}{2}\left(\boldsymbol{v}_{\alpha, i, j, k}^{p}\right)^{2}, \frac{1}{v_{\alpha, i}^{*, p}} \frac{{\overline{\left(\tilde{f}_{\alpha}^{p+1}\right)_{i, j+\frac{1}{2}, k}^{\text {central }}}}^{{ }^{\prime}} \overline{\left(\tilde{f}_{\alpha}^{p+1}\right)_{i, j-\frac{1}{2}, k}^{\text {central }}}}{\Delta c_{\|}}\right\rangle_{\delta \boldsymbol{c} \text { where } v_{\|, \alpha, i, j+\frac{1}{2}}<u_{\|, \alpha, i}^{p}} .
\end{aligned}
$$

Thus we see Eq. E.17) is the discrete time derivative of the total fluid energy of the system, including the total thermal and kinetic energy of the plasma. Recalling the definition of $E_{\|, i}^{p+1}$ we may rearrange the summation in Eq. E.17), which gives us

$$
\begin{aligned}
\delta_{t} U_{\text {fluid }}-\sum_{\alpha}^{N_{s p}} \sum_{i}^{N_{x}} \Delta x\left\{\bar{\Gamma}_{\|, \alpha, i+\frac{1}{2}}^{p+1} E_{\|, i+\frac{1}{2}}^{p+1} q_{\alpha}\right\} & \\
& \quad-\sum_{\alpha}^{N_{s p}} m_{\alpha} \sum_{i}^{N_{x}} \Delta x\left\{\left(\phi_{\alpha, i}^{+, p+1}+1\right) \Gamma_{\|, \alpha, i}^{+, p+1}+\left(\gamma_{q, \alpha, i}^{-, p+1}+1\right) \Gamma_{\|, \alpha, i}^{-, p+1}\right\}=0,
\end{aligned}
$$

where we defined

$$
\bar{\Gamma}_{\|, \alpha, i+\frac{1}{2}}^{p+1} \equiv \frac{\bar{\Gamma}_{\|, \alpha, i}^{p+1}+\bar{\Gamma}_{\|, \alpha, i+1}^{p+1}}{2} .
$$

Next, we recall the symmetry with Ampère's equation in Eq. (B.17) and define

$$
\bar{\Gamma}_{\|, \alpha, i+\frac{1}{2}}^{p+1}=\widehat{\Gamma}_{\|, \alpha, i+\frac{1}{2}}^{p+1}
$$

to enforce particle fluxes in the current in Ampère's equation that come from the energy moment of the acceleration operator. Thus, we find that the nonlinear constraint function $\xi_{\alpha}$ is completely determined by requiring

$$
\xi_{\alpha, i+\frac{1}{2}}^{p+1}=\frac{\bar{\Gamma}_{\|, \alpha, i+\frac{1}{2}}^{p+1}-\widetilde{\Gamma}_{\|, \alpha, i+\frac{1}{2}}^{p+1}}{\Pi_{\xi, \|, \alpha, i+\frac{1}{2}}^{p+1}} .
$$


We now observe that Eq. E.20 must become

$$
\begin{aligned}
& \delta_{t} U_{\text {fluid }}+\sum_{i}^{N_{x}} \Delta x\left\{\epsilon_{0} E_{\|, i+\frac{1}{2}}^{p+1} \delta_{t} E_{\|, i+\frac{1}{2}}-E_{\|, i+\frac{1}{2}}^{p+1} \bar{j}_{\|}^{p+1}\right\} \\
&-\sum_{i}^{N_{x}} \Delta x\left\{\sum_{\alpha}^{N_{s p}} m_{\alpha}\left[\left(\phi_{\alpha, i}^{+, p+1}+1\right) \Gamma_{\|, \alpha, i}^{+, p+1}+\left(\gamma_{q, \alpha, i}^{-, p+1}+1\right) \Gamma_{\|, \alpha, i}^{-, p+1}\right]\right\}=0 .
\end{aligned}
$$

In a previous implementation of this method, as was done in Refs. [20, 30], the use of a Crank-Nicolson integration scheme ensured the equivalence

$$
E_{\|, i+\frac{1}{2}}^{p+1} \delta_{t} E_{\|, i+\frac{1}{2}}=\frac{1}{2} \delta_{t}\left(E_{\|, i+\frac{1}{2}}^{2}\right)
$$

in the discrete. However, BDF2 in the current development does not ensure this relation. Thus, the second purpose of the quantities $\phi$ and $\gamma_{q}$ is to enforce the equivalence of Eq. (E.24) to the equation

$$
\begin{aligned}
\delta_{t} U_{\text {fluid }}+\sum_{i}^{N_{x}} \Delta x\left\{\delta_{t}\left(\epsilon_{0} \frac{1}{2} E_{\|, i+\frac{1}{2}}^{2}\right)-E_{\|, i+\frac{1}{2}}^{p+1} \bar{j}_{\|}^{p+1}\right\} & \\
& =\delta_{t} U_{\text {fluid }}+\delta_{t} U_{E_{\|}}-\bar{E}_{\|}^{p+1} \bar{j}_{\|}^{p+1}=\delta_{t} U_{\text {total }}-\bar{E}_{\|}^{p+1} \bar{j}_{\|}^{p+1}=0 .
\end{aligned}
$$

Note, that in the absence of an external electric field, $\bar{E}_{\|}^{p+1} \bar{j}_{\|}^{p+1}$ vanishes discretely, preserving the discrete conservation principle. The final constraint on $\phi$ and $\gamma_{q}$ is thus

$$
\left(\phi_{\alpha, i}^{+, p+1}+1\right) \Gamma_{\|, \alpha, i}^{+, p+1}+\left(\gamma_{q, \alpha, i}^{-, p+1}+1\right) \Gamma_{\|, \alpha, i}^{-, p+1}=\frac{1}{m_{\alpha} N_{s p}}\left\{\epsilon_{0}\left[E_{\|, i+\frac{1}{2}}^{p+1} \delta_{t} E_{\|, i+\frac{1}{2}}-\delta_{t}\left(\frac{1}{2} E_{\|, i+\frac{1}{2}}^{2}\right)\right]\right\}
$$

where we have pulled the temporal derivatives into the species summation by dividing by the number of species, $N_{s p}$.

We see from Eqs. E.15) and E.26) that $\phi$ and $\gamma_{q}$ are determined for each species and locally at each point in the configuration space by a simple $2 \times 2$ linear system of equations:

$$
\left[\begin{array}{cc}
n_{\alpha, i}^{+, p+1} & n_{\alpha, i}^{-, p+1} \\
\Gamma_{\|, p, i}^{+, p+1} & \Gamma_{\|, \alpha, i}^{-, p+1}
\end{array}\right]\left[\begin{array}{c}
\left(\phi_{\alpha, i}^{+, p+1}+1\right. \\
\left(\gamma_{q, \alpha, i}^{-, p+1}+1\right.
\end{array}\right]=\left[\begin{array}{c}
\left(n_{\alpha, i}^{p+1}-\bar{n}_{\alpha, i}^{p+1}\right) E_{\|, i}^{p+1} \frac{q_{\alpha}}{m_{\alpha}} \\
\frac{1}{m_{\alpha} N_{s p}}\left\{\epsilon_{0}\left[E_{\|, i+\frac{1}{2}}^{p+1} \delta_{t} E_{\|, i+\frac{1}{2}}-\delta_{t}\left(\frac{1}{2} E_{\|, i+\frac{1}{2}}^{2}\right)\right]\right\}
\end{array}\right] .
$$

To ensure solvability of Eq. (E.27), the determinant of the system

$$
\operatorname{Det}\left[\begin{array}{cc}
n_{\alpha, i}^{+, p+1} & n_{\alpha, i}^{-, p+1} \\
\Gamma_{\|, \alpha, i}^{+, p+1} & \Gamma_{\|, \alpha, i}^{-, p+1}
\end{array}\right]=n_{\alpha, i}^{+, p+1} \Gamma_{\|, \alpha, i}^{-, p+1}-n_{\alpha, i}^{-, p+1} \Gamma_{\|, \alpha, i}^{+, p+1}
$$

must be strictly finite. It can be shown that if the splitting velocity for Eq. E.10) is within the discrete bounds of $v_{\|, \alpha, i, j}^{p+1}$ then the system in Eq. E.27) is well-posed (see Appendix F. To reduce nonlinearity of the algorithm we use $u_{\|, \alpha, i}^{p}$ as the splitting velocity, which is sufficiently close to $u_{\|, \alpha, i}^{p+1}$ for the constraint functions $\phi$ and $\gamma_{q}$ to remain well-behaved.

\section{Appendix F. Well-posedness of $2 \times 2$ system for $\phi^{+}$and $\gamma_{q}^{-}$}

In Sec. 4 we presented a $2 \times 2$ linear system to be solved (locally, for each species) for $\phi_{\alpha, i}^{-, p+1}$ and $\gamma_{q, \alpha, i}^{-, p+1}$. This may be expressed in simpler notation as

$$
\left[\begin{array}{ll}
n^{+} & n^{-} \\
\Gamma^{+} & \Gamma^{-}
\end{array}\right]\left[\begin{array}{c}
\left(\phi^{+}+1\right) \\
\left(\gamma_{q}^{-}+1\right)
\end{array}\right]=\left[\begin{array}{l}
R_{1} \\
R_{2}
\end{array}\right]
$$

To be well-posed, the determinant

$$
\operatorname{Det}\left[\begin{array}{ll}
n^{+} & n^{-} \\
\Gamma^{+} & \Gamma^{-}
\end{array}\right]=n^{+} \Gamma^{-}-n^{-} \Gamma^{+}
$$


must be strictly non-zero. To show when this is the case, we will for simplicity consider a 1D-1V system, with moments $n$ and $n u$ defined as

$$
\begin{aligned}
& n=\int_{-\infty}^{\infty} f(v) d v \\
& \Gamma=\int_{-\infty}^{\infty} v f(v) d v
\end{aligned}
$$

We define the bulk velocity $u \equiv \frac{\Gamma}{n}$, and the split quantities $n^{+/-}$and $\Gamma^{+/-}$by

$$
\begin{aligned}
& n^{-}=\int_{-\infty}^{u^{*}} f(v) d v \\
& n^{+}=\int_{u^{*}}^{\infty} f(v) d v \\
& \Gamma^{-}=\int_{-\infty}^{u^{*}} v f(v) d v \\
& \Gamma^{+}=\int_{u^{*}}^{\infty} v f(v) d v .
\end{aligned}
$$

We may then perform a coordinate transformation $v^{\prime}=v-u^{*}$ of the integration for Eqs. (F.7) and [F.8):

$$
\begin{aligned}
& \Gamma^{-}=\int_{-\infty}^{0}\left(v^{\prime}+u^{*}\right) f\left(v^{\prime}\right) d v^{\prime}=\int_{-\infty}^{0} v^{\prime} f\left(v^{\prime}\right) d v^{\prime}+n^{-} u^{*} \\
& \Gamma^{+}=\int_{0}^{\infty}\left(v^{\prime}+u^{*}\right) f\left(v^{\prime}\right) d v^{\prime}=\int_{0}^{\infty} v^{\prime} f\left(v^{\prime}\right) d v^{\prime}+n^{+} u^{*}
\end{aligned}
$$

If we define

$$
\begin{aligned}
& (n w)^{-}=\int_{-\infty}^{0} v^{\prime} f\left(v^{\prime}\right) d v^{\prime}, \\
& (n w)^{+}=\int_{0}^{\infty} v^{\prime} f\left(v^{\prime}\right) d v^{\prime}
\end{aligned}
$$

we see that $(n w)^{-}$is negative definite and $(n w)^{+}$is positive definite. Equation F.2 now becomes

$$
n^{+}\left[(n w)^{-}+n^{-} u^{*}\right]-n^{-}\left[(n w)^{+}+n^{+} u^{*}\right]=n^{+}(n w)^{-}-n^{-}(n w)^{+}+n^{+} n^{-}\left(u^{*}-u^{*}\right),
$$

which is negative definite. Thus, we find that Eq. (F.1) is well-posed for arbitrary (finite) $u^{*}$. We note that in the discrete system $u_{\alpha, i}^{*}$ must lie within the discrete bounds of $v_{\|}$for the given species $\alpha$ at configuration-space index $i$. In practice any choice near $u_{\|, \alpha, i}^{p+1}$ should be suitable.

\section{Appendix G. Two-stream instability for cold Maxwellian beams}

Recall that the general dispersion relation for the electron-electron two stream instability [49] is

$$
1+\frac{\omega_{p, b}^{2}}{k^{2} v_{t h, b}^{2}}\left[2+\zeta_{+} Z\left(\zeta_{+}\right)+\zeta_{-} Z\left(\zeta_{-}\right)\right]=0
$$

where

$$
\zeta_{ \pm} \equiv \frac{\omega \mp k v_{b}}{k v_{t h, b}}
$$

and $\omega_{p, b}$ is the beam plasma frequency. Recall also that if the electron beams are delta functions (i.e. in the limit $v_{t h, b} \rightarrow 0$ ) Eq. (G.1) becomes 


$$
1-\frac{1}{\left(\omega+v_{b} k\right)^{2}}-\frac{1}{\left(\omega-v_{b} k\right)^{2}}=0 .
$$

Based on the delta-function dispersion relation, Eq. (G.2), the growth rate of electric field energy is $\gamma=0.353 \omega_{p, b}$. However, as we mentioned in Sec. (6.2), for thermalized beams there will be some deviation, and we expect that as the ratio $v_{t h, b} / v_{b}$ increases that the system will become more stable (i.e. $\gamma$ will decrease). Figure 4 indeed shows that if we increase $v_{t h, b} / v_{b}$ towards some critical ratio near 1, the growth rate decreases precipitously. Here we present a semi-analytic analysis of the generalized two-stream dispersion relation in Eq. (G.1).

First, we will rearrange Eq. G.1):

$$
k^{2} \lambda_{D}^{2}+2+\zeta_{+} Z\left(\zeta_{+}\right)+\zeta_{-} Z\left(\zeta_{-}\right)=0,
$$

where $\lambda_{D}^{2} \equiv \frac{v_{t h, b}^{2}}{\omega_{p, b}^{2}}$. For convenience we then recast $\zeta_{ \pm}$in terms of dimensionless quantities:

$$
\zeta_{ \pm}=\left(\frac{\beta_{r}}{\delta} \mp \alpha\right)+i\left(-\frac{\beta}{\delta}\right)
$$

where

$$
\begin{gathered}
\omega \equiv \omega_{r}-i \gamma, \alpha \equiv \frac{v_{b}}{v_{t h, b}}, \\
\beta_{r} \equiv \frac{\omega_{r}}{\omega_{p, b}}, \beta \equiv \frac{\gamma}{\omega_{p, b}}, \\
\delta \equiv k \lambda_{D} .
\end{gathered}
$$

We next observe that the plasma dispersion function $Z(\zeta)$ is given as

$$
Z(\zeta)=\frac{1}{\sqrt{\pi}} \int_{-\infty}^{\infty} \frac{e^{-z^{2}}}{z-\zeta} d z
$$

which may be expressed in terms of the complex error function erf $(z)$ as [47]

$$
Z(\zeta)=i \sqrt{\pi} e^{-\zeta^{2}}[1+\operatorname{erf}(i \zeta)]
$$

Thus, combining Eqs. G.4 and G.6) with Eq. G.3, we obtain

$$
\begin{aligned}
\delta^{2}+2+i \sqrt{\pi} \zeta_{+} e^{-\left[\left(\frac{\beta_{r}}{\delta}-\alpha\right)+i\left(-\frac{\beta}{\delta}\right)\right]^{2}} & {\left[1+\operatorname{erf}\left(i\left(\frac{\beta_{r}}{\delta}-\alpha\right)-\left(-\frac{\beta}{\delta}\right)\right)\right] } \\
+ & i \sqrt{\pi} \zeta_{-} e^{-\left[\left(\frac{\beta_{r}}{\delta}+\alpha\right)+i\left(-\frac{\beta}{\delta}\right)\right]^{2}}\left[1+\operatorname{erf}\left(i\left(\frac{\beta_{r}}{\delta}+\alpha\right)-\left(-\frac{\beta}{\delta}\right)\right)\right]=0 .
\end{aligned}
$$

Equation (G.7) may be separated into its real and imaginary components, and for a given $\alpha$ and $\delta$ (i.e., given the beam velocity $v_{b}$, beam thermal speed $v_{t h, b}$, wavenumber $k$, and Debye length $\lambda_{D}$ ), we may solve for the instability growth rate $\beta$ (as well as the oscillatory component $\beta_{r}$ ).

For the case in Sec. 6.2. we have $1 / \alpha=v_{t h, b} / v_{b}=[0.15,0.3,0.5,0.65,0.8]$ and $k=2 \pi$. For this case, we have $\omega_{p, b}=\omega_{p, e}=1$, defined using the total electron density, which with a fixed $\left|v_{b}\right|=0.1$, will give us $\delta=$ $2 \pi \times[0.015,0.03,0.05,0.065,0.08]$. As we saw in Table 1 , this will produce

$$
\beta=[0.3488,0.3318,0.2734,0.1953,0.08911] .
$$

\section{References}

[1] Hans G. Rinderknecht, P. A. Amendt, S. C. Wilks, and G. Collins. Kinetic physics in ICF: present understanding and future directions. Plasma Physics and Controlled Fusion, 60(064001), 2018. 
[2] W. T. Taitano, A. N. Simakov, L. Chacón, and B. D. Keenan. Yield degradation in inertial-confinement-fusion implosions due to shock-driven kinetic fuel-species stratification and viscous heating. Physics of Plasmas, 25(056310), 2018.

[3] Brett D. Keenan, Andrei N. Simakov, William T. Taitano, and Luis Chacón. Ion species stratification within strong shocks in two-ion plasmas. Physics of Plasmas, 25(3), 2018.

[4] O. Larroche, H. G. Rinderknecht, M. J. Rosenberg, N. M. Hoffman, S. Atzeni, R. D. Petrasso, P. A. Amendt, and F. H. Séguin. Ion-kinetic simulations of D- 3 He gas-filled inertial confinement fusion target implosions with moderate to large Knudsen number. Physics of Plasmas, 23(012701), 2016.

[5] H. G. Rinderknecht, P. A. Amendt, M. J. Rosenberg, C. K. Li, J. A. Frenje, M. Gatu Johnson, H. Sio, F. H. Séguin, R. D. Petrasso, A. B. Zylstra, G. Kagan, N. M. Hoffman, D. Svyatsky, S. C. Wilks, V. Yu. Glebov, C. Stoeckl, and T. C. Sangster. Ion kinetic dynamics in strongly-shocked plasmas relevant to ICF. Nuclear Fusion, 57(066014), 2017.

[6] W. T. Taitano, L. Chacón, and A. N. Simakov. An adaptive, implicit, conservative, 1D-2V multi-species VlasovFokker-Planck multi-scale solver in planar geometry. Journal of Computational Physics, 365:173-205, 2018.

[7] O. Larroche. Kinetic simulations of fuel ion transport in ICF target implosions. European Physical Journal D, 27(2):131-146, 2003.

[8] J. F. Luciani, P. Mora, and J. Virmont. Nonlocal heat transport due to steep temperature gradients. Physical Review Letters, 51(18):1664-1667, 1983.

[9] G. P. Schurtz, P. D. Nicolaï, and M. Busquet. A nonlocal electron conduction model for multidimensional radiation hydrodynamics codes. Physics of Plasmas, 7(10):4238-4249, 2000.

[10] W. T. Taitano, L. Chacón, A. N. Simakov, and K. Molvig. A mass, momentum, and energy conserving, fully implicit, scalable algorithm for the multi-dimensional, multi-species Rosenbluth-Fokker-Planck equation. Journal of Computational Physics, 297:357-380, 2015.

[11] W. T. Taitano, L. Chacón, and A. N. Simakov. An adaptive, conservative 0D-2V multispecies RosenbluthFokker-Planck solver for arbitrarily disparate mass and temperature regimes. Journal of Computational Physics, 318:391-420, 2016.

[12] W. T. Taitano, L. Chacón, and A. N. Simakov. An equilibrium-preserving discretization for the nonlinear Rosenbluth-Fokker-Planck operator in arbitrary multi-dimensional geometry. Journal of Computational Physics, 339:453-460, 2017.

[13] William T. Taitano, Dana A Knoll, Luis Chacón, and Guangye Chen. Development of a consistent and stable fully implicit moment method for Vlasov-Ampere particle in cell (PIC) system. SIAM Journal on Scientific Computing, 35(5):126-149, 2013.

[14] G. Chen, L. Chacón, and D. C. Barnes. An energy- and charge-conserving, implicit, electrostatic particle-in-cell algorithm. Journal of Computational Physics, 230:7018-7036, 2011.

[15] G. Chen, L. Chacón, C. A. Leibs, D. A. Knoll, and W. T. Taitano. Fluid preconditioning for Newton-Krylovbased, fully implicit, electrostatic particle-in-cell simulations. Journal of Computational Physics, pages 555-$567,2014$.

[16] G. Chen and L. Chacón. A multi-dimensional, energy- and charge-conserving, nonlinearly implicit, electromagnetic Vlasov-Darwin particle-in-cell algorithm. Computer Physics Communications, 197:73-87, 2015.

[17] James A. Rossmanith and David C. Seal. A positivity-preserving high-order semi-Lagrangian discontinuous Galerkin scheme for the Vlasov-Poisson equations. Journal of Computational Physics, 230:6203-6232, 2011.

[18] Nicolas Besse and E. Sonnendrücker. Semi-Lagrangian schemes for the Vlasov equation on an unstructured mesh of phase space. Journal of Computational Physics, 191(2):341-376, nov 2003. 
[19] C. Z. Cheng and Georg Knorr. The integration of the vlasov equation in configuration space. Journal of Computational Physics, 22(3):330-351, 1976.

[20] William T. Taitano and Luis Chacón. Charge-and-energy conserving moment-based accelerator for a multispecies Vlasov-Fokker-Planck-Ampère system, part I: Collisionless aspects. Journal of Computational Physics, 284:718-736, 2015.

[21] Richard B. Horne and Mervyn P. Freeman. A New Code for Electrostatic Simulation by Numerical Integration of the Vlasov and Ampère Equations Using MacCormack's Method. Journal of Computational Physics, 171:182200, 2001.

[22] Yingda Cheng, Andrew J. Christlieb, and Xinghui Zhong. Energy-conserving discontinuous Galerkin methods for the Vlasov-Maxwell system. Journal of Computational Physics, 279:145-173, 2014.

[23] Sebastiano Boscarino, Francis Filbet, and Giovanni Russo. High Order Semi-implicit Schemes for Time Dependent Partial Differential Equations. Journal of Scientific Computing, 68:975-1001, 2016.

[24] Pierre Degond and Fabrice Deluzet. Asymptotic-Preserving methods and multiscale models for plasma physics. Journal of Computational Physics, 336:429-457, may 2017.

[25] Alina Chertock, Changhui Tan, and Bokai Yan. An asymptotic preserving scheme for kinetic models with singular limit. Kinetic and Related Models, 11(4):735-756, apr 2018.

[26] L. Chacón, G. Chen, D. A. Knoll, C. Newman, H. Park, W. T. Taitano, J. A. Willert, and G. Womeldorff. Multiscale high-order/low-order (HOLO) algorithms and applications. Journal of Computational Physics, 330:21-45, 2017.

[27] D. A. Knoll, H. Park, and Kord Smith. Application of the Jacobian-Free Newton-Krylov Method to Nonlinear Acceleration of Transport Source Iteration in Slab Geometry. Nuclear Science and Engineering, 167:122_-132, 2011.

[28] H. Park, D. A. Knoll, R. M. Rauenzahn, A. B. Wollaber, and J. D. Densmore. A Consistent, Moment-Based, Multiscale Solution Approach for Thermal Radiative Transfer Problems. Transport Theory and Statistical Physics, 41:284--303, 2012.

[29] William T. Taitano, Dana A. Knoll, Luis Chacón, Jon M. Reisner, and Anil K. Prinja. Moment-based acceleration for neutral gas kinetics with BGK collision operator. Journal of Computational and Theoretical Transport, 43:83-108, 2014.

[30] William T. Taitano, Dana A. Knoll, and Luis Chacón. Charge-and-energy conserving moment-based accelerator for a multi-species Vlasov-Fokker-Planck-Ampère system, part II: Collisional aspects. Journal of Computational Physics, 284:737-757, 2015.

[31] William T. Taitano, Luis Chacón, Andrei N. Simakov, and Steven E. Anderson. A conservative phase-space moving-grid strategy for a 1D-2V Vlasov-Fokker-Planck Equation. eprint arXiv:1903.05467, 2019.

[32] Francis Filbet and Thomas Rey. A rescaling velocity method for dissipative kinetic equations: Applications to granular media. Journal of Computational Physics, 248:177-199, sep 2013.

[33] P. J. Morrison. Structure and structure-preserving algorithms for plasma physics. Physics of Plasmas, 24(055502), 2017.

[34] Takashi Shiroto, Naofumi Ohnishi, and Yasuhiko Sentoku. Quadratic conservative scheme for relativistic VlasovMaxwell system. Journal of Computational Physics, 379:32-50, 2019.

[35] P. H. Gaskell and A. K.C. Lau. Curvature-compensated convective transport: SMART, A new boundednesspreserving transport algorithm. International Journal for Numerical Methods in Fluids, 8:617-641, 1988.

[36] Guang-Shan Jiang and Chi-Wang Shu. Efficient implementation of weighted ENO schemes. Journal of Computational Physics, 126:202-228, 1996. 
[37] P. J. Mardahl and J. P. Verboncoeur. Charge conservation in electromagnetic PIC codes; spectral comparison of Boris/DADI and Langdon-Marder methods. Computer Physics Communications, 106(3):219-229, 1997.

[38] Barry Marder. A method for incorporating Gauss' law into electromagnetic PIC codes. Journal of Computational Physics, 68:48-55, 1987.

[39] John Villasenor and Oscar Buneman. Rigorous charge conservation for local electromagnetic field solvers. Computer Physics Communications, 69:306-316, 1992.

[40] Yuxi Chen and Gábor Tóth. Gauss's Law satisfying Energy-Conserving Semi-Implicit Particle-in-Cell method. Journal of Computational Physics, 386:632-652, 2019.

[41] E. Hairer, S. P. Nørsett, and G. Wanner. Solving Ordinary Differential Equations I: Nonstiff Problems. SpringerVerlag, Berlin, 2nd edition, 2008.

[42] B. P. Leonard. A stable and accurate convective modelling procedure based on quadratic upstream interpolation. Computer Methods in Applied Mechanics and Engineering, 19:59-98, 1979.

[43] Homer F. Walker and Peng Ni. Anderson Acceleration for Fixed-Point Iterations. SIAM Journal on Numerical Analysis, 49(4):1715--1735, 2011.

[44] Donald G. Anderson. Iterative Procedures for Nonlinear Integral Equations. Journal of the ACM, 12(4):547-560, 1965.

[45] Jeffrey Willert, William T. Taitano, and Dana Knoll. Leveraging Anderson Acceleration for improved convergence of iterative solutions to transport systems. Journal of Computational Physics, 273:278-286, 2014.

[46] Youcef Saad. A Flexible Inner-Outer Preconditioned GMRES Algorithm. SIAM Journal on Scientific Computing, 14(2):461-469, 1993.

[47] B. D. Fried and S. D. Conte. The Plasma Dispersion Function. Academic Press Inc., New York, NY, 1961.

[48] T. H. Watanabe and H. Sugama. Vlasov and drift kinetic simulation methods based on the symplectic integrator. Transport Theory and Statistical Physics, 34:287-309, 2005.

[49] Francis F. Chen. Introduction to Plasma Physics and Controlled Fusion, Volume 1: Plasma Physics, volume 53. Plenum Press, 2 edition, 1989.

[50] M. A. Shay, J. F. Drake, and B. Dorland. Equation Free Projective Integration: A multiscale method applied to a plasma ion acoustic wave. Journal of Computational Physics, 226:571—-585, 2007. 